AperTO - Archivio Istituzionale Open Access dell'Università di Torino

\title{
Spectrum Estimates and Applications to Geometry
}

\section{This is a pre print version of the following article:}

Original Citation:

\section{Availability:}

This version is available http://hdl.handle.net/2318/1693257 since 2019-02-18T20:42:09Z

Publisher:

Atlantic Press

Published version:

DOI:10.2991/978-94-6239-240-3_7

Terms of use:

Open Access

Anyone can freely access the full text of works made available as "Open Access". Works made available under a Creative Commons license can be used according to the terms and conditions of said license. Use of all other works requires consent of the right holder (author or publisher) if not exempted from copyright protection by the applicable law. 


\title{
SPECTRUM ESTIMATES AND APPLICATIONS TO GEOMETRY
}

\author{
G. PACELLI BESSA, L. JORGE, L. MARI AND J. FÁBIO MONTENEGRO
}

\begin{abstract}
Mathematics Subject Classification (2000): 58C40, 53C42
Key words: Spectrum, Laplacian, Cheeger's constant, foliations, submersions, eigenvalues, Calabi-Yau, minimal surface, density.
\end{abstract}

\section{INTRODUCTION}

In 1867, E. Beltrami [14] introduced a second order elliptic operator on Riemannian manifolds, defined by $\Delta=\operatorname{div} \circ$ grad, extending the Laplace operator on $\mathbb{R}^{n}$, called the Laplace-Beltrami operator. The Laplace-Beltrami operator became one of the most important operators in Mathematics and Physics, playing a fundamental role in differential geometry, geometric analysis, partial differential equations, probability, potential theory, stochastic process, just to mention a few. It is in important in various differential equations that describe physical phenomena such as the diffusion equation for the heat and fluid flow, wave propagation, Laplace equation and minimal surfaces.

An important step towards the analysis of this operator was taken by M. Gaffney [66] in 1954, proving that, for geodesically complete metrics, the Laplace-Beltrami operator is essentially self-adjoint, meaning that it has a unique self-adjoint extension, denoted also by $\Delta$, whose domain is the set of functions $f \in L^{2}$ so that $\Delta f \in L^{2}$. If a Riemannian manifold $M$ is geodesically incomplete, there exist infinitely many self-adjoint extensions, but just one whose domain lies in that of the associated quadratic form, called the Friedrichs extension of $\left(\Delta, C_{0}^{\infty}(M)\right)$. The spectrum of these self-adjoint extensions is formed by all $\lambda \in[0, \infty)$ for which $(\Delta+\lambda I)$ is not injective or the inverse operator $(\Delta+\lambda I)^{-1}$ is unbounded, see [50] for a detailed account.

The spectrum of the Laplace-Beltrami operator encodes fundamental properties of the geometry of the underline Riemannian manifold, and has various applications highlighted in see [15], [29], [33], [34], [49], [50], [71], [73], and also [135]. The problems involving spectrum of the Laplace-Beltrami operator (Laplacian for short) vary in aspect, taste and difficulty. In this manuscript we will address basic questions about the nature of the spectrum as a by-product of the geometry of the underlining Riemannian manifold. The content gives our trajectory in the study of the geometry of the Laplacian on manifolds and submanifolds in the last fifteen years. Our study starts with basic estimates of the bottom of the spectrum then progressing to questions about the whole spectrum.

We will refer to the spectrum of the Laplacian as the spectrum of $\Omega$ or $M$ and denote it by $\sigma(\Omega)$ or $\sigma(M)$. It is important in our description to distinguish the types of elements in the spectrum $\sigma(M)$. The set of $\lambda$ for which $(\Delta+\lambda I)$ is not injective is the set of all eigenvalues of $\sigma(M)$ and it is called the point spectrum $\sigma_{p}(M)$, while the discrete spectrum $\sigma_{d}(M) \subset \sigma_{p}(M)$ is the set of all isolated eigenvalues of finite multiplicity. The complement of the discrete spectrum is the essential spectrum, $\sigma_{\text {ess }}(M)=\sigma(M) \backslash \sigma_{d}(M)$ and the complement of the point spectrum is the continuous spectrum, $\sigma_{\text {cont }}(M)=\sigma(M) \backslash \sigma_{p}(M)$. To have a glimpse of these kind of questions, see [54], [56], [61], [85], [119], [131] for geometric conditions implying that the spectrum is purely continuous, $\sigma_{p}(M)=\emptyset$ or these [8], [53], [78], [86], [87] implying the spectrum is discrete, $\sigma_{\text {ess }}(M)=\emptyset$. 
The manuscript is organized as follows. In Section 2, we study the bottom of the spectrum inf $\sigma(M)$ of a given Riemannian manifold, in terms of geometric invariants. This section is divided in two parts. In the first part, subsection 2.1, we introduce a geometric and a computable, non-negative constant $c(M)^{1}$ in order to give a lower bound for the bottom of the spectrum of a Riemannian manifold $M$. We show that $\inf \sigma(M) \geq c(M)^{2} / 4$, where $c(M)=\sup _{X}[\inf \operatorname{div} X / \sup |X|], X$ smooth vector fields in $M$, see [21]. This lower bound allow us to obtain explicitly the Cheeger's constant for geodesic balls in model manifolds [24], to study foliations of spaces by constant mean curvature leaves in terms of the bottom of the spectrum of these spaces and to prove a Haymann-Makai-Osserman inequality for tubes around curves in $\mathbb{R}^{n}$, see [11]. In the second part, subsection 2.5 , we extend Barta's theorem, proved for bounded open sets, to all open subsets of Riemannian manifolds. The main application of this generalization is a geometric lower bound of the spectrum of minimal submanifolds in terms of the ambient sectional curvature.

Section 4 is an application of the estimates developed in Section 2 to study the spectrum of certain Riemannian submersions. More precisely, we study the spectrum of Riemannian submersions $\pi: M \rightarrow N$ with compact minimal fibers $F \rightarrow M$. The main result is that for a Riemannian submersion $\pi: M \rightarrow N$ with compact minimal fibers, each type of the spectrum of $N$ is contained in the respective spectrum of the total space. This is, $\sigma_{p}(N) \subset \sigma_{p}(M), \sigma_{\text {ess }}(N) \subset \sigma_{\text {ess }}(N)$ and $\inf \sigma_{\text {ess }}(N)=\inf \sigma_{\text {ess }}(M)$, therefore $M$ has discrete spectrum if and only if $N$ has discrete spectrum.

In Section 5, we consider the Calabi-Yau conjectures of minimal hypersurfaces set in Yau's Millennium Lectures [138], [139]. S. T. Yau, revisiting the E. Calabi conjectures on the existence of bounded minimal hypersurfaces, [30], [44], after the Jorge-Xavier and Nadirashvili's counter-examples, [84], [107], proposed a new set of questions about bounded minimal surfaces of $\mathbb{R}^{3}$. He wrote: "It is known [107] that there are complete minimal surfaces properly immersed into the [open] ball. What is the geometry of these surfaces? Can they be embedded? Since the curvature must tend to minus infinity, it is important to find the precise asymptotic behaviour of these surfaces near their ends. Are their [Laplacian] spectra discrete?". In this chapter we give a fairly complete answer to this question, proving that complete bounded minimal surfaces has discrete spectrum provided the dimension of its limit set is small. Our main result applies to a number of examples recently constructed, and is sharp.

In Section 6 is, in some sense, the opposite of Section 5. There, we investigate conditions to guarantee that a half-line is contained in $\sigma_{\text {ess }}(M)$. In some instances, notably for minimal submanifolds $M^{m} \rightarrow \mathbb{N}_{\kappa}^{n}$ of space forms, we are able to exhibit sharp conditions to ensure that $\sigma(M)$ is a half-line. These conditions might involve the density function of $M$ or the behavious of geodesic balls in $M$. Applications include the investigation of the family of examples of complete minimal surfaces between parallel planes of Jorge-Xavier and Rosenberg-Toubiana.

Hereafter, for a given Riemannian manifold $M$ we write $\Delta: \mathcal{D}(\Delta) \rightarrow L^{2}(M)$ to denote the self-adjoint extension of the Laplace-Beltrami operator if $M$ is geodesically complete, or otherwise the Friedrichs extension of $\left(\Delta, C_{0}^{\infty}(M)\right)$.

\section{Fundamental tone estimates}

If $M$ is compact then $\sigma(M)=\left\{\lambda_{1}(M)<\lambda_{2}(M) \leq \cdots \nearrow \nearrow_{\infty}\right.$, with the eigenvalues repeated accordingly to their multiplicities. If $\partial M=\emptyset$ then $\lambda_{1}(M)=0$ and if $\partial M \neq \emptyset$ then $\lambda_{1}(M)>0$. To describe the spectrum of a given Riemannian manifold is a hard problem although the spectrum in few cases is known. For instance, if $\mathbb{S}_{\kappa}^{n}, \mathbb{R}^{n}, \mathbb{H}_{\kappa}^{n}$ denote

\footnotetext{
${ }^{1} c(M)>0$ if $M$ is compact with non-empty boundary
} 
the space forms of curvature $\kappa>0,0$ and $-\kappa<0$, respectively, then

$$
\left\{\begin{array}{l}
\sigma\left(\mathbb{R}^{n}\right)=[0, \infty) \\
\sigma\left(\mathbb{H}_{\kappa}^{n}\right)=\left[\frac{(n-1)^{2} \kappa}{4}, \infty\right) \\
\sigma\left(\mathbb{S}_{1}^{n}\right)=\left\{\nu_{j}=j(j+n-1)\right\} \quad j=0,1, \ldots, \infty,
\end{array}\right.
$$

while $\lambda_{1}\left(B_{\mathbb{R}^{n}}(r)\right)=c^{2}(n) / r^{2}, \lambda_{1}\left(B_{\mathbb{S}^{n}}(\pi / 2)\right)=n$, see [33].

The bottom of the spectrum, $\inf \sigma(M)$ and of the essential spectrum $\inf \sigma_{\text {ess }}(M)$ of $M$ can be characterized in terms of fundamental tones of open subsets $\Omega \subseteq M$. More precisely, $\inf \sigma(M)=\lambda^{*}(M)$ and $\inf \sigma_{\mathrm{ess}}(M)=\sup _{K \subset M} \lambda^{*}(M \backslash K), K \subset M$ compact, see [50], [53], [114, Thm.21], where the fundamental tone $\lambda^{*}(\Omega)$ of an open set $\Omega \subseteq M$ is defined by

$$
\lambda^{*}(\Omega)=\inf \left\{\frac{\int_{\Omega}|\nabla u|^{2}}{\int_{\Omega} u^{2}}, u \in C_{0}^{\infty}(\Omega) \backslash\{0\}\right\} .
$$

Furthermore, $\lambda^{*}(M \backslash K)$ is the bottom of the spectrum of the Friedrichs extension of $\left(-\Delta, C_{0}^{\infty}(M \backslash K)\right)$. If $\Omega$ is relatively compact then $\lambda^{*}(\Omega)=\lambda_{1}(\Omega)$ coincides with the first Dirichlet eigenvalue $\lambda_{1}(\Omega)$ of $\Omega$, see [33].

The very basic question posed by Schoen-Yau in [125] is: what are the geometries with positive spectrum? The positivity $\lambda^{*}(M)>0$ imposes strong restrictions on the geometry of $M$, for instance, $M$ is non-parabolic, that is, it admits non-constant positive superharmonic functions, see [71, Prop. 10.1]. The converse statement is not true. The Euclidean space $\mathbb{R}^{n}$ is non-parabolic for $n \geq 3$ and $\lambda^{*}\left(\mathbb{R}^{n}\right)=0$ for all $n \geq 1$. Letting $V(r)=\operatorname{vol}\left(B_{M}(r)\right)$ be the volume of the geodesic ball of radius $r$ then if $V(r) \leq p(r)$, for any polynomial $p(r)$ and $r>0$ then $\lambda^{*}(M)=0$, [42]. Cheng-Yau's result was extended by R. Brooks and then by Y. Higuchi in $[29,79]$. They proved that

$$
\inf \sigma_{\text {ess }}(M) \leq \frac{\tau^{2}(M)}{4}, \quad \text { where } \quad \tau(M)=\liminf _{r \rightarrow \infty} \frac{\log V(r)}{r}
$$

is the volume entropy. These results mentioned give interesting geometric consequences of $\lambda^{*}(M)>0$, however, it would be interesting to know how positive the bottom of the spectrum is. In other words, give precise lower bounds for $\lambda^{*}(M)$ in terms of geometric invariants. For instance, the classic McKean's Theorem [104] gives sharp lower bound for the bottom of the spectrum of Hadamard manifolds with negative curvature.

Theorem 2.1 (McKean-[104]). Let $M$ be a complete, simply connected, Riemannian $n$ manifold with sectional curvature $K_{M} \leq-\kappa<0$. The bottom of the spectrum of $M$ is bounded below as

$$
\lambda^{*}(M) \geq \frac{(n-1)^{2} \kappa}{4} .
$$

\section{Remark 2.2.}

- This lower bound is sharp since inf $\sigma\left(\mathbb{H}_{\kappa}^{n}\right)=(n-1)^{2} \kappa / 4$.

- The curvature assumption $K_{M} \leq-\kappa<0$ in Theorem 2.1 is necessary in the sense that if there is a family of disjoint, flat geodesic balls $B_{M}\left(r_{i}\right)$ with center at $x_{i}$ and radii $r_{i} \rightarrow \infty$ them inf $\sigma(M)=\inf \sigma_{\text {ess }}(M)=0$ regardless the sectional curvature behaviour in $M \backslash\left(\cup_{i=1}^{\infty} B_{M}\left(r_{i}\right)\right)$, see Definition 6.11 in Section 5.

The fundamental tone $\lambda^{*}(M)$ of a geodesically complete Riemannian manifold $M$ given in (2) can be also obtained as the $\operatorname{limit}_{r \rightarrow \infty} \lambda^{*}\left(B_{M}(r)\right)=\lim \lambda^{*}(M)$, where $B_{M}(r)$ is the geodesic ball with radius $r$ and center at a point $p$. The following result is known as 
the Cheng's Eigenvalue Comparison Theorem a local version of McKean's Theorem, see [39].

Theorem 2.3 (S. Y. Cheng). Let $M$ be a Riemannian n-manifold and let $B_{M}(r)$ be a normal geodesic ball of radius $r$. Let $\kappa=\sup _{B_{M}(r)} K$ be the supremum of all the sectional curvatures of $B_{M}(r)$. If $\kappa>0$, assume further that $r<\pi / 2 \sqrt{\kappa}$. Then

$$
\lambda^{*}\left(B_{M}(r)\right) \geq \lambda^{*}\left(B_{\mathbb{M}_{\kappa}^{n}}(r)\right) .
$$

Here $\mathbb{M}_{\kappa}^{n}$ is the simply connected $n$-space form of constant sectional curvature $\kappa$.

A theoretical lower bound estimate for $\lambda^{*}(\Omega)$, for relatively compact open sets $\Omega$ with smooth boundary $\partial \Omega$ was obtained by J. Cheeger, in [34]. He introduced what is now called Cheeger's constant $h(\Omega)$ defined by

$$
h(\Omega)=\inf _{A \subset \Omega} \frac{\operatorname{vol}_{n-1}(\partial A)}{\operatorname{vol}_{n}(A)},
$$

where $A \subset \Omega$ is connected, and proved that

$$
\lambda^{*}(\Omega) \geq \frac{h^{2}(\Omega)}{4} .
$$

Taking an exhaustion of $M$ by relatively compact open sets $\left\{\Omega_{i}\right\}$ with smooth boundaries one readily has that $\lambda^{*}(M) \geq h^{2}(M) / 4$. The relevance of Cheeger's lower bound (5) is that it relates the first eigenvalue $\lambda^{*}(\Omega)$ with the isoperimetric inequality (4). However, it is difficult to give lower bounds for fundamental tones via Cheeger's constant because it is hardy computable. In the next subsection will introduce a constant, that can be estimated, and used to give lower bounds for the fundamental tones of open sets. This constant is defined in terms of divergence of vector fields and it has great flexibility in the geometric applications. For instance, in the particular cases of geodesic balls of model manifolds, with center at the pole, we show that our constant coincide with Cheeger's constant.

2.1. Lower bound estimates and geometric applications. The purpose of this subsection is to describe a criterion for a lower bound on $\lambda^{*}(\Omega)$ in terms of possibily non-smooth vector fields. We begin with the following

Definition 2.4. Let $M$ be a Riemannian manifold and a vector field $X \in L_{l o c}^{1}(M)$ (meaning that $|X| \in L_{\text {loc }}^{1}(M)$ ). A function $g \in L_{\text {loc }}^{1}(M)$ is a weak divergence of $X$ if

$$
\int_{M} \phi g=-\int_{M}\langle\nabla \phi, X\rangle, \forall \phi \in C_{0}^{\infty}(M) .
$$

There exists at most one weak divergence $g \in L_{l o c}^{1}(M)$ for a given $X \in L_{l o c}^{1}(M)$ and we may write $g=\operatorname{Div} X$. For $C^{1}$ vector fields $X$ the classical (strong) divergence $\operatorname{div} X$ and the weak divergence $\operatorname{Div} X$ coincide.

Remark 2.5. Let $\mathcal{W}^{1,1}(M)$ denote the space of all vector fields $X \in L_{l o c}^{1}(M)$ possessing weak divergence $\operatorname{Div} X$. If $X \in \mathcal{W}^{1,1}(M)$ and $f \in C^{1}(M)$ then $f X \in \mathcal{W}^{1,1}(M)$ with

$$
\operatorname{Div}(f X)=\langle\nabla f, X\rangle+f \operatorname{Div} X .
$$

In particular for $f \in C_{0}^{\infty}(M)$ we have that

$$
\int_{M} \operatorname{Div}(f X)=\int_{M}\langle\nabla f, X\rangle-\langle\nabla f, X\rangle=0
$$


Conversely, if $f X \in \mathcal{W}^{1,1}(M)$ for all $f \in C_{0}^{\infty}(M)$ then $X \in \mathcal{W}^{1,1}(M)$. In this case

$$
\operatorname{Div}(X)=\sum_{i=1}^{\infty} \operatorname{Div}\left(\xi_{i} X\right)
$$

where $\left\{\xi_{i}\right\}$ is a partition of unity subordinated to a locally finite covering of $M$ by open sets.

Remark 2.6. The gradient $X=\nabla \rho_{M}$ of the distance function $\rho_{M}(x)=\operatorname{dist}_{M}(p, x)$ to a point $p \in M$ fails to be smooth in $p$ and in cut $(p)$, the cut locus of $p$. However, it is possible to show that $\nabla \rho_{M} \in \mathcal{W}^{1,1}(M)$ provided the $(n-1)$-Hausdorff measure $\mathcal{H}^{n-1}(\operatorname{cut}(p))=0$. This justifies to work in this larger class $\mathcal{W}^{1,1}(M)$ of vector fields. It is interesting to observe that, combining work of various authors (one is referred to the account in Chapter 1 of [26]), $\mathcal{H}^{n-1}(\operatorname{cut}(p))=0$ if and only if $\operatorname{Cut}(p)$ consists just of focal points.

Definition 2.7. Let $\Omega \subset M$ be an open subset of a Riemannian manifold $M$. Let $\mathcal{X}(\Omega)$ be defined by

$$
\mathcal{X}(\Omega)=\left\{X \in \mathcal{W}^{1,1}(\Omega): \sup _{\Omega}|X|<\infty, \inf _{\Omega} \operatorname{Div} X>0\right\} .
$$

Define $c(\Omega)$ by

$$
c(\Omega)=\sup _{X \in \mathcal{X}(\Omega)} \frac{\inf _{\Omega} \operatorname{Div} X}{\sup _{\Omega}|X|} .
$$

Our first result of this section is this following lower bound for fundamental tones of open sets obtained in [21].

Theorem 2.8 (Bessa-Montenegro-[21]). Let $\Omega \subset$ be an open subset of a Riemannian manifold $M$. Then

$$
\lambda^{*}(\Omega) \geq \frac{c(\Omega)^{2}}{4} .
$$

Proof. Let $X \in \mathcal{X}(\Omega)$ and $f \in C_{0}^{\infty}(\Omega)$. The vector field $f^{2} X \in \mathcal{X}(\Omega)$. Computing Div $f^{2} X$ we have,

$$
\begin{aligned}
\operatorname{Div}\left(f^{2} X\right) & =\left\langle\nabla f^{2}, X\right\rangle+f^{2} \operatorname{Div}(X) \\
& \geq-\left|\nabla f^{2}\right| \cdot|X|+\inf _{\Omega} \operatorname{Div} X \cdot f^{2} \\
& \geq-2 \cdot \sup _{\Omega}|X| \cdot|f| \cdot|\nabla f|+\inf _{\Omega} \operatorname{Div} X \cdot f^{2}
\end{aligned}
$$

Using the inequality

$$
-2 \cdot|f| \cdot|\nabla f| \geq-\epsilon \cdot|f|^{2}-1 / \epsilon \cdot|\nabla f|^{2}
$$

for all $\epsilon>0$, we have from (9) that

$$
\operatorname{Div}\left(f^{2} X\right) \geq \sup _{\Omega}|X| \cdot\left(-\epsilon \cdot|f|^{2}-\frac{1}{\epsilon} \cdot|\nabla f|^{2}\right)+\inf _{\Omega} \operatorname{Div} X \cdot f^{2}
$$

Integrating (10) on a normal domain $\mathcal{O}$ containing $\Omega$ we have that

$$
0=\int_{\mathcal{O}} \operatorname{Div}\left(f^{2} X\right) \geq \sup _{\Omega}|X| \cdot \int_{\mathcal{O}}\left(-\epsilon|f|^{2}-\frac{1}{\epsilon}|\nabla f|^{2}\right)+\inf _{\Omega} \operatorname{Div} X \cdot \int_{\mathcal{O}} f^{2},
$$

therefore

$$
\int_{\mathcal{O}}|\nabla f|^{2} \geq \frac{\epsilon}{\sup _{\Omega}|X|}\left(\inf _{\Omega} \operatorname{Div} X-\sup _{\Omega}|X| \cdot \epsilon\right) \int_{\mathcal{O}} f^{2}
$$


Choosing $\epsilon=\left(\inf _{\Omega} \operatorname{Div} X\right) /\left(2 \sup _{\Omega}|X|\right)$ we have that

$$
\int_{\Omega}|\nabla f|^{2}=\int_{\mathcal{O}}|\nabla f|^{2} \geq\left[\frac{\inf _{\Omega} \operatorname{Div} X}{2 \sup _{\Omega}|X|}\right]^{2} \int_{\mathcal{O}} f^{2}=\left[\frac{\inf _{\Omega} \operatorname{Div} X}{2 \sup _{\Omega}|X|}\right]^{2} \int_{\Omega} f^{2} .
$$

Then, by fundamental tone definition (2), inequality (11) implies that

$$
\lambda(\Omega) \geq\left(\frac{\inf _{\Omega} \operatorname{Div} X}{2 \sup _{\Omega}|X|}\right)^{2} .
$$

Taking, in the right hand side of (12), the supremum over all vector fields $X \in \mathcal{X}(\Omega)$ we have inequality (8).

Remark 2.9. For relatively compact open sets with smooth boundaries, it can be shown that $c(\Omega) \leq h(\Omega)$, where $h(\Omega)=\inf _{A \subset \Omega} \operatorname{Vol}_{n-1}(\partial A) / \operatorname{Vol}_{n}(A)$ is Cheeger's constant for $\Omega$. To see that, let $X \in \mathcal{X}(\Omega)$ and $A \subset \Omega$ be a connected open subset of $\Omega$. Then we have that

$$
\inf _{\Omega} \operatorname{Div} X \cdot \operatorname{Vol}_{n}(A) \leq \int_{A} \operatorname{Div} X=\int_{\partial A}\langle X, \eta\rangle \leq \sup _{\Omega}|X| \cdot \operatorname{Vol}_{n-1}(\partial A) .
$$

Thus,

$$
\frac{\inf _{\Omega} \operatorname{Div} X}{\sup _{\Omega}|X|} \leq \frac{\operatorname{Vol}_{n-1}(\partial A)}{\operatorname{Vol}_{n}(A)}
$$

The left and right sides of inequality (14) are independent on each other. Therefore, taking the supremum on the right hand side and infimum on the left hand side one has that $c(\Omega) \leq h(\Omega)$. The advantage of introducing $c(\Omega)$ is the easiest computability compared with $h(\Omega)$.

Corollary 2.10 (Cheng's revisited). Let $M$ be a Riemannian n-manifold and let $B_{M}(r)$ be a normal geodesic ball of radius $r$. Let $\kappa=\sup _{B_{M}(r)} K$ be the supremum of all the sectional curvatures of $B_{M}(r)$. If $\kappa>0$, assume further that $r<\pi / 2 \sqrt{\kappa}$. Then,

$$
\lambda^{*}\left(B_{N}(r)\right) \geq \begin{cases}\max \left\{\frac{4 n^{2}}{r^{2}}, \frac{[(n-1) k \operatorname{coth}(k r)]^{2}}{4}\right\}, & \text { if } \quad \kappa=-k^{2} . \\ \frac{n^{2}}{4 r^{2}}, & \text { if } \quad \kappa=0 . \\ \frac{[(n-1) \cdot k r \cot (k r)+1]^{2}}{4 r^{2}}, & \text { if } \quad \kappa=k^{2} \text { and } r<\pi / 2 k .\end{cases}
$$

2.2. Cheeger constant of model manifolds. Let $h \in C^{2}([0,+\infty))$ be positive in $\left(0, R_{h}\right), 0<R_{h} \leq \infty$ satisfying $h(0)=0, h^{\prime}(0)=1$. The $n$-dimensional model $\mathbb{M}_{h}^{n}$ is the manifold $\mathbb{R}^{n}$ endowed with a metric whose expression, in polar coordinates $(t, \theta)$ centered at some origin $o$, reads

$$
\mathrm{d} s_{h}^{2}=\mathrm{d} t^{2}+h(t)^{2}\langle,\rangle_{\mathbb{S}^{n-1}},
$$

where $\langle,\rangle_{\mathbb{S}^{n-1}}$ is the standard metric on the unit $(n-1)$-sphere. The metric can be extended in a $C^{2}$-way if $h^{\prime \prime}(0)=0$, and smoothly if $h \in C^{\infty}([0,+\infty))$ and $h^{2 j}(0)=0$ for each $j \in \mathbb{N}$, see [69]. Observe that a model $\mathbb{M}_{h}^{n}$ can, equivalently, be specified by prescribing its radial sectional curvature $G \in C^{\infty}\left(\mathbb{R}_{0}^{+}\right)$and recovering $h$ as the solution of

$$
\left\{\begin{array}{l}
h^{\prime \prime}-G h=0, \\
h(0)=0, h^{\prime}(0)=1,
\end{array}\right.
$$

on the maximal interval $\left(0, R_{h}\right)$ where $h>0$. 
Remark 2.11. Denoting by $G_{-}$the negative part of $G$, i.e. $G_{-}=\max \{0,-G\}$, then $R_{g}=+\infty$ whenever $t \int_{t}^{\infty} G_{-}(s) d s \leq 1 / 4$, see $[26]$.

A model manifold enjoys the following properties:

- The Laplace-Beltrami operator on $\mathbb{M}_{h}^{n}$ written in polar coordinates is

$$
\Delta=\frac{\partial^{2}}{\partial t}+(n-1) \frac{h^{\prime}}{h} \frac{\partial}{\partial t}+\frac{1}{h^{2}} \Delta_{\mathbb{S}^{n-1}} .
$$

- The volume of the geodesic ball $B_{\mathbb{M}_{h}}(r)$ and the volume of the geodesic sphere $\partial B_{\mathbb{M}_{h}}(r)$ centered at the origin are given respectively by

$$
\operatorname{Vol}_{n}\left(B_{\mathbb{M}_{h}}(r)\right)=\omega_{n} \int_{0}^{r} h^{n-1}(s) d s \text { and } \operatorname{Vol}_{n-1}\left(\partial B_{\mathbb{M}_{h}}(r)\right)=\omega_{n} h^{n-1}(r) .
$$

Where $\omega_{n}$ is the volume of the unit sphere $\mathbb{S}^{n-1}$.

The space form $\mathbb{M}_{\kappa}^{n}$ of curvature $\kappa>0,0$ or $-\kappa<0$ are recovered by the choice $h=S_{\kappa}$, where

$$
S_{\kappa}(t)=\left\{\begin{array}{ccc}
\frac{\sin (\sqrt{\kappa} t)}{\sqrt{\kappa}}, & \text { if } & \kappa>0 \\
t, & \text { if } & \kappa=0 \\
\frac{\sinh (\sqrt{-\kappa} t)}{\sqrt{-\kappa}}, & \text { if } & \kappa<0
\end{array}\right.
$$

Volumes of geodesic spheres and balls in $\mathbb{M}_{\kappa}^{n}$ will be denoted with $v_{\kappa}(r)$ and $V_{\kappa}(r)$, respectively.

Our first task is to characterize the Cheeger's constant of models:

Theorem 2.12 (Bessa-Montenegro-[24]). Let $B_{\mathbb{M}_{h}}(r)$ be a geodesic ball centered at the origin of a model manifold $\mathbb{M}_{h}$ then

$$
c\left(B_{\mathbb{M}_{h}}(r)\right)=h\left(B_{\mathbb{M}_{h}}(r)\right) .
$$

In particular,

$$
\lambda^{*}\left(B_{\mathbb{M}_{h}}(r)\right) \geq \inf _{0 \leq t \leq r}\left[\frac{\operatorname{Vol}_{n-1}\left(\partial B_{\mathbb{M}_{h}}(t)\right)}{2 \operatorname{Vol}_{n}\left(B_{\mathbb{M}_{h}}(t)\right)}\right]^{2} .
$$

Proof. Consider the function $E: B_{\mathbb{M}_{h}}(r) \rightarrow \mathbb{R}$ defined by

$$
E(x)=\int_{r(x)}^{r} \frac{1}{h^{n-1}(\tau)} \int_{0}^{\tau} h^{n-1}(s) d s d \tau
$$

Observe that $E$ is a radial function, meaning that $E(x)=E(r(x)), r(x)=\operatorname{dist}_{\mathbb{M}_{h}}(0, x)$. This function is called the mean exit time function [71]. Letting $X=-\nabla E$ we easily compute that $\operatorname{div} X=-\Delta E=1$ and

$$
|X|(t, \theta)=\frac{1}{h^{n-1}(t)} \int_{0}^{t} h^{n-1}(s) d s=\frac{\operatorname{Vol}\left(B_{\mathbb{M}_{h}}(t)\right)}{\operatorname{Vol}\left(\partial B_{\mathbb{M}_{h}}(t)\right)} .
$$


By (4) and by (7) we have that

$$
\begin{aligned}
c\left(B_{\mathbb{M}_{h}}(r)\right) & \geq \frac{1}{\sup _{0 \leq t \leq r} \frac{\operatorname{Vol}\left(B_{\mathbb{M}_{h}}(t)\right)}{\operatorname{Vol}\left(\partial B_{\mathbb{M}_{h}}(t)\right)}} \\
& =\inf _{0 \leq t \leq r} \frac{\operatorname{Vol}_{n-1}\left(\partial B_{\mathbb{M}_{h}}(t)\right)}{\operatorname{Vol}_{n}\left(B_{\mathbb{M}_{h}}(t)\right)} \\
& \geq h\left(B_{\mathbb{M}_{h}}(r)\right) .
\end{aligned}
$$

However, $\left.\left.h\left(B_{\mathbb{M}_{h}}(r)\right)\right) \geq c\left(B_{\mathbb{M}_{h}}(r)\right)\right)$, as observed in Remark 2.9. This shows that

$$
c\left(B_{\mathbb{M}_{h}}(r)\right)=h\left(B_{\mathbb{M}_{h}}(r)\right) .
$$

Example 2.13. The Cheeger constants for geodesic balls in the sphere $\mathbb{S}^{2}$, Euclidean space $\mathbb{R}^{2}$ and hyperbolic space $\mathbb{H}^{2}$ are respectively

$$
\left\{\begin{array}{l}
h\left(B_{\mathbb{R}^{2}}(r)\right)=\frac{2}{r} \text { and } h\left(\mathbb{R}^{2}\right)=0 \\
h\left(B_{\mathbb{S}^{2}}(r)\right)=\frac{\sin (r)}{1-\cos (r)} \text { and } h\left(B_{\mathbb{S}^{2}}(\pi / 2)\right)=1, \quad h\left(\mathbb{S}^{2}\right)=0 \\
h\left(B_{\mathbb{H}^{2}}(r)\right)=\frac{\sinh (r)}{\cosh (r)-1} \text { and } h\left(\mathbb{H}^{2}\right)=1 .
\end{array}\right.
$$

It is worthy to observe that the eigenvalue estimate via Cheeger constant sometimes is too coarse. For instance, it is well know that $\lambda_{1}\left(B_{\mathbb{S}^{2}}(\pi / 2)\right)=2$ and $h\left(B_{\mathbb{S}^{2}}(\pi / 2)\right)=1$.

2.3. Transversally oriented foliations. Let $\Omega$ be an open set of a Riemannian manifold $\mathrm{M}$ and $\mathcal{F}$ a codimension one transversally oriented $C^{3}$-foliation. This means that we may choose a smooth unit vector field $\eta$ on $M$ that is normal to the leaves of $\mathcal{F}$. Let $H^{F}(x)$ be the value of the mean curvature of the leaf $F$ at $x$ computed with respect to $\eta$. Set $b=\inf _{F \in \mathcal{F}} \inf _{x \in F}\left|H^{F}(x)\right|$. Assume first that $b>0$. This implies that $H^{F}$ does not change sign. Hence, we may choose the unit vector field $\eta$ in such way that $H^{F}(x)>0$ for any $x \in \Omega$. It is easy to compute $\operatorname{div}_{M} \eta=n \cdot H^{F}$. Therefore, $\inf _{\Omega} \operatorname{diver}_{M} \eta=n \cdot \inf _{\Omega} H_{F}(x) \geq n \cdot b$. Since $|\eta|=1$, by (8), we have the estimate

$$
2 \sqrt{\lambda^{*}(\Omega)} \geq \inf _{\Omega} \frac{\operatorname{div} \eta}{\|\eta\|_{\infty}}=n \cdot \inf _{x \in \Omega} H_{F}(x) \geq n \cdot b .
$$

This proves the following result, see [11].

Theorem 2.14 (Barbosa-Bessa-Montenegro-[11]). Let $\Omega$ be a connected open subset of Riemannian $(n+1)$-manifold $M$ admitting a transversely oriented codimension one $C^{2}$ foliation $\mathcal{F}$. Then

$$
2 \sqrt{\lambda^{*}(\Omega)} \geq n \cdot \inf _{F \in \mathcal{F}} \inf _{x \in F}\left|H^{F}(x)\right|,
$$

where $H^{F}$ stands for the mean curvature function of the leaf $F$.

This theorem has a number of consequences, stated below as corollaries. It imposes restrictions for the existence of foliations by constant mean curvature hypersurfaces on open sets with zero fundamental tone or on open sets with Ricci curvature bounded below, see Corollaries 2.15 and 2.17 . 
Corollary 2.15. Let $\mathcal{F}$ be a transversely oriented codimension one $C^{2}$-foliation of a Riemannian manifold $M$ for which $\lambda^{*}(M)=0$. If the leaves of $\mathcal{F}$ have the same constant mean curvature then they are minimal submanifolds of $M$.

Remark 2.16. The class of Riemannian manifolds $M$ with $\lambda^{*}(M)=0$ is huge. Besides the compact Riemannian manifolds, it contains all the open Riemannian manifolds with asymptotically nonnegative Ricci curvature, see [38]. An open Riemannian manifold $M$ has asymptotically nonnegative Ricci curvature if $\operatorname{Ric}_{M}(x) \geq-\psi\left(\operatorname{dist}_{M}\left(x_{0}, x\right)\right)$, for a continuous function $\psi:[0, \infty) \rightarrow[0, \infty)$ with $\lim _{t \rightarrow \infty} \psi(t)=0, x_{0} \in M$. In fact, the class contains the Riemannian manifolds with a disjoint family of flat balls described in Remark 2.2.

In [12], Barbosa-Kenmotsu-Oshikiri considered transversally oriented codimension-one $C^{3}$-foliations $\mathcal{F}$ of the simply connected space form $\mathbb{M}_{\kappa}^{n}$ of curvature $-\kappa \leq 0$. They proved that if the leaves were complete oriented hypersurfaces with the same constant mean curvature $H \geq(n-1) \sqrt{\kappa}$ then $H=(n-1) \sqrt{\kappa}$. The next result extends BarbosaKenmotsu-Oshikiri's theorem to Riemannian $n$-manifolds with Ricci curvature $\operatorname{Ric}_{M} \geq$ $-(n-1) \kappa$.

Corollary 2.17. Let $\mathcal{F}$ be a transversely oriented codimension-one $C^{2}$-foliation of a complete $n$-dimensional Riemannian manifold $M$ with Ricci curvature $\operatorname{Ric}_{M} \geq-(n-1) \kappa$, for some $\kappa \geq 0$. Then

i) $2 \sqrt{\lambda^{*}\left(\mathbb{M}_{\kappa}^{n}\right)} \geq \inf _{F \in \mathcal{F}} \inf _{x \in F}\left|H_{F}(x)\right|$, where $\mathbb{M}_{\kappa}^{n}$ is the simply connected $n$-space form $\mathbb{M}_{\kappa}^{n}$ of constant curvature $-\kappa \leq 0$.

ii) If $\left|H_{F}\right| \geq b>0$ then $(n-1) \sqrt{\kappa} \geq b$.

Proof. Let $B_{M}(r)$ be a geodesic ball of radius $r$ of a Riemannian Manifold $M$ whose Ricci curvature satisfies $\operatorname{Ric}_{M} \geq-(n-1) \kappa$, and let $B_{\mathbb{M}_{\kappa}^{n}}(r)$ be the geodesic ball of radius $r$ in the model $n$-manifold $\mathbb{M}_{\kappa}^{n}$ of constant sectional curvature $-\kappa$. By Cheng's Comparison Theorem, [38] we know that

$$
\lambda^{*}\left(B_{M}(r)\right) \leq \lambda^{*}\left(B_{\mathbb{M}_{\kappa}^{n}}(r)\right) .
$$

Since $\lambda^{*}(M)=\lim _{r \rightarrow \infty} \lambda^{*}\left(B_{M}(r)\right)$, it follows that $\lambda^{*}(M) \leq \lambda^{*}\left(\mathbb{M}_{\kappa}^{n}\right)$. Thus, by Theorem 2.14 we have

$$
\inf _{F \in \mathcal{F}} \inf _{x \in F}\left|H_{F}(x)\right| \leq 2 \sqrt{\lambda^{*}(M)} \leq 2 \sqrt{\lambda^{*}\left(\mathbb{M}_{\kappa}^{n}\right)}
$$

and $(i)$ is proved. Item $(i i)$ follows immediately by using that $\lambda^{*}\left(\mathbb{M}_{\kappa}^{n}\right)=(n-1)^{2} \kappa / 4$. This completes the proof of the corollary.

Theorem 2.14 also has a version for scalar curvature provided the ambient manifold has nonpositive sectional curvature.

Corollary 2.18. Let $M$ be a Riemannian $(n+1)$-manifold with nonpositive sectional curvature $K_{M} \leq 0$ and let $\mathcal{F}$ be a transversely oriented codimension one $C^{2}$-foliation of a connected open set $\Omega \subset M$. Suppose that the scalar curvature $S_{F}$ of each leaf if nonnegative. Then

$$
\sqrt{\inf S} \leq 2 \sqrt{\lambda^{*}(\Omega)}
$$

In particular, if $\lambda^{*}(M)=0$ and all the leaves have the same constant non-negative scalar curvature $S \geq 0$, then $S=0$.

If inf $S=0$ there is nothing to prove. Thus, we assume that inf $S=c>0$. Let $p \in F$ and $\left\{e_{1}, \cdots, e_{n}\right\}$ be an orthonormal basis for the tangent space $T_{p} F$ of the leaf $F \in \mathcal{F}$. The Gauss equation for the plane generated by $e_{i}, e_{j}$ is:

$$
\tilde{K}\left(e_{i}, e_{j}\right)=\bar{K}\left(e_{i}, e_{j}\right)+\left\langle B\left(e_{i}, e_{i}\right), B\left(e_{j}, e_{j}\right)\right\rangle-\left|B\left(e_{i}, e_{j}\right)\right|^{2},
$$


where $\widetilde{K}$ is the Gaussian curvature of $F$ and $\bar{K}$ is the Gaussian curvature of the ambient space $\Omega \times \mathbb{R}$. Tracing on $i, j$ gives:

$$
S(p)=\sum_{i, j} \bar{K}\left(e_{i}, e_{j}\right)+n^{2} H^{2}-\|B\|^{2} .
$$

Since the sectional curvatures $\bar{K} \leq 0$, it follows that

$$
S(p) \leq n^{2} H^{2}
$$

and, since $S \geq c>0$ then $H \geq \sqrt{c} / n>0$. By Theorem 2.14 we have that

$$
2 \sqrt{\lambda^{*}(\Omega)} \geq n \inf _{F \in \mathcal{F}} \inf _{x \in F}\left|H_{f}(x)\right| \geq \sqrt{c}=\sqrt{\inf S} .
$$

This proves Corollary 2.18.

We turn to higher order mean curvatures. If $\psi: N \rightarrow M$ is an $n$-dimensional oriented hypersurface of $M$ and $k_{1}, \ldots, k_{n}$ are the principal curvatures at $p \in N$, then the $r$-th mean curvatures $H_{r}$ of $\psi(N)$ at $\psi(p)$ are defined by the identity

$$
\left(1+t k_{1}\right)\left(1+t k_{2}\right) \cdots\left(1+t k_{n}\right)=1+\left(\begin{array}{c}
n \\
1
\end{array}\right) H_{1} t+\left(\begin{array}{c}
n \\
2
\end{array}\right) H_{2} t^{2}+\cdots\left(\begin{array}{c}
n \\
n
\end{array}\right) H_{n} t^{n}
$$

for all real number $t$. Thus, $H_{1}$ is the mean curvature of $\psi, H_{n}$ is the Gauss-Kronecker curvature. Since we always have $H_{1}^{2} \geq H_{2}$, the following version of Theorem (2.14) for the 2-nd mean curvature $H_{2}$ is direct.

Corollary 2.19. Let $\mathcal{F}$ be a transversely oriented codimension one $C^{2}$-foliation of a connected open set $\Omega$ of a Riemannian manifold $M$. Suppose that the leaves have the 2-nd mean curvature $H_{2} \geq 0$. Then

$$
2 \sqrt{\lambda^{*}(\Omega)} \geq n \cdot \inf _{F \in \mathcal{F}} \inf _{x \in F}\left(H_{2}^{F}\right)^{1 / 2}(x),
$$

where $H_{2}^{F}$ stands for the second mean curvature function of the leaf $F$. In particular, if $\lambda^{*}(M)=0, \Omega=M$, and all the leaves have the same constant 2-nd mean curvature $H_{2} \geq 0$ then $H_{2}=0$.

2.4. Haymann-Makai-Osserman inequality. Recall that the inradius $\rho(\Omega)$ of a connected open set $\Omega$ of a Riemannian manifold $M$ is defined as $\rho(\Omega)=\sup \left\{r>0 ; B_{M}(r) \subset\right.$ $\Omega\}$, where $B_{M}(r)$ is a geodesic ball of radius $r$ of $M$. In [96], Makai proved that the fundamental tone $\lambda^{*}(\Omega)$ of a simply connected bounded domain $\Omega \subset \mathbb{R}^{2}$ with inradius $\rho$ and smooth boundary is bounded below by $\lambda_{1}(\Omega) \geq 1 / 4 \rho^{2}$. Unaware of Makai's result, Haymann [77] proved years later that $\lambda_{1}(\Omega) \geq 1 / 900 \rho^{2}$. Osserman [112] among other things improved Haymann's estimate back to $\lambda_{1}(\Omega) \geq 1 / 4 \rho^{2}$. Recently, Haymann-MakaiOsserman inequality was improved by Bañuelos-Carroll in [9] to $0.6197 / \rho^{2}$.

In the next result, we prove Haymann-Makai-Osserman inequality to embedded tubular neighbourhoods of simple smooth curves in $\mathbb{R}^{n}$ with variable radius.

Theorem 2.20. Let $\gamma: I=(\alpha, \beta) \subset \mathbb{R} \rightarrow \mathbb{R}^{n}$ be a simple smooth curve and $T_{\gamma}(\rho(t))$ be an embedded tubular neighborhood of $\gamma$ with variable radius $\rho(t)$ and smooth boundary $\partial T_{\gamma}(\rho(t))$. Let $\rho_{o}=\sup _{t} \rho(t)>0$ be its inradius. Then

$$
\lambda^{*}\left(T_{\gamma}(\rho(t))\right) \geq \frac{(n-1)^{2}}{4 \rho_{o}^{2}} .
$$


Consider the family of balls of $\mathbb{R}^{n} B_{t}=B_{\mathbb{R}^{n}}\left(\gamma(t), \rho_{o}\right), t \in I$ with center at $\gamma(t)$ and radius $\rho_{o}$. The set $\left\{S_{t}=\partial B_{t} \cap T_{\gamma}(\rho(t))\right\}$ is a smooth codimension one transversally oriented foliation of $T_{\gamma}(\rho(t)) \backslash\left(B_{\alpha} \cup B_{\beta}\right)$. Pushing the family $B_{t}$ little further one can fill $T_{\gamma}(\rho(t))$ with a smooth codimension one transversally oriented foliation such that the mean curvature of the leaves is constant $1 / \rho_{o}$. Thus, we have by Theorem 2.14 that $\lambda^{*}\left(T_{\gamma}(\rho)\right) \geq(n-1)^{2} / 4 \rho^{2}$.

2.5. Barta's Theorem generalized. A simple but effective method to obtain lower and upper bounds on the first Dirichlet eigenvalue $\lambda_{1}(\Omega)$ of bounded open subsets $\Omega \subset M$ with piecewise smooth boundaries ${ }^{2}$ of Riemannian manifolds was obtained by J. Barta in [13].

Theorem 2.21 (Barta). Let $\Omega \subset M$ be a compact subset of a Riemannian manifold $M$, with piecewise smooth non-empty boundary $\partial \Omega$ and $f \in C^{2}(\Omega) \cap C^{0}(\bar{\Omega})$ with $f \mid \Omega>0$ and $f \mid \partial \Omega=0$ and $\lambda_{1}(\Omega)$ be the first Dirichlet eigenvalue of $\Omega$. Then

$$
\sup _{\Omega}(-\Delta f / f) \geq \lambda_{1}(\Omega) \geq \inf _{\Omega}(-\Delta f / f) .
$$

With equality in (24) if and only if $f$ is a first eigenfunction of $\Omega$.

\section{Remark 2.22.}

- To obtain the lower bound for $\lambda_{1}(M)$ we may suppose only that $f \mid \partial \Omega \geq 0$.

- It is hard to obtain a non-trivial upper bound for $\lambda_{1}(\Omega)$ via Barta's Theorem, because to make a meaningful estimate when $f \mid \partial \Omega=0$ one also has to have that $\Delta f \mid \partial \Omega=0$.

Our main result in this section is a refinement of Theorem 2.8, i.e. we give better lower bounds for the fundamental tones of arbitrary open sets $\Omega$ in terms of divergence of smooth vector fields $X$. When $X=-\nabla \log f$ for a positive smooth function $f \mid \Omega>0$ then our lower bound becomes $\inf (-\Delta f / f)$. This is, our result can be viewed as a generalization of Barta's Theorem.

Theorem 2.23 (Bessa-Montenegro-[23]). Let $\Omega \subset M$ be a open subset of a Riemannian manifold. The fundamental tone $\lambda^{*}(\Omega)$ is bounded below as

$$
\lambda^{*}(\Omega) \geq \sup _{X \in \mathcal{W}^{1,1}(\Omega)}\left\{\inf _{\Omega}\left(\operatorname{Div} X-|X|^{2}\right)\right\} .
$$

If $\Omega$ is compact with boundary then

$$
\lambda_{1}(\Omega)=\sup _{\mathcal{W}^{1,1}}\left\{\inf _{M}\left(\operatorname{Div} X-|X|^{2}\right)\right\} .
$$

Remark 2.24. If $X=-\nabla \log (v)$, for a positive smooth function of $v: \Omega \rightarrow \mathbb{R}$ we obtain that $\operatorname{div} X-|X|^{2}=-(\Delta v / v)$. Thus $\lambda^{*}(\Omega) \geq \inf _{\Omega}(-\Delta v / v)$. In particular, if $\Omega$ is relatively compact with boundary and $v$ is a positive first eigenfunction then $\lambda^{*}(\Omega)=(-\Delta v / v)$.

Proof. The proof is a variation of the one of Theorem 2.8. Let $X \in \mathcal{W}^{1,1}(\Omega)$ and $f \in$ $C_{0}^{\infty}(\Omega)$. The vector field $f^{2} X \in \mathcal{X}(\Omega)$. Computing $\operatorname{Div} f^{2} X$ we have,

$$
\begin{aligned}
\operatorname{Div}\left(f^{2} X\right) & =\left\langle\nabla f^{2}, X\right\rangle+f^{2} \operatorname{Div}(X) \\
& \geq-\left|\nabla f^{2}\right| \cdot|X|+\operatorname{Div} X \cdot f^{2} \\
& \geq-2 \cdot \sup _{\Omega}|X| \cdot|f| \cdot|\nabla f|+\operatorname{Div} X \cdot f^{2} \\
& \geq-|\nabla f|^{2}+\left(\operatorname{Div} X-|X|^{2}\right) \cdot f^{2}
\end{aligned}
$$

\footnotetext{
${ }^{2}$ Piecewise smooth boundary here means that there is a closed set $Q \subset \partial M$ of $(n-1)$-Hausdorff measure zero such that for each point $q \in \partial M \backslash Q$ there is a neighborhood of $q$ in $\partial M$ that is a graph of a smooth function over the tangent space $T_{q} \partial M$, see Whitney [136] pages 99-100.
} 
Integrating (27) on $\Omega$ we have that

$$
0=\int_{\Omega} \operatorname{Div}\left(f^{2} X\right) \geq-\int_{\Omega}|\nabla f|^{2}+\inf _{\Omega}\left(\operatorname{Div} X-|X|^{2}\right) \int_{\Omega} f^{2}
$$

Therefore $\frac{\int_{\Omega}|\nabla f|^{2}}{\int_{\Omega} f^{2}} \geq \inf _{\Omega}\left(\operatorname{Div} X-|X|^{2}\right)$. Taking the infimum over all $f \in C_{0}^{\infty}(\Omega)$ and the supremum over all $X \in \mathcal{W}^{1,1}(\Omega)$ implies that

$$
\lambda^{*}(\Omega) \geq \sup _{X \in \mathcal{W}^{1,1}(\Omega)} \inf _{\Omega}\left(\operatorname{Div} X-|X|^{2}\right) .
$$

This proves Theorem 2.23.

The following lemma gives sufficient conditions to a given vector field $X$ to belong to $\mathcal{W}^{1,1}(\Omega)$. Its proof can be found in [22, Lemma 3.1]

Lemma 2.25. Let $\Omega \subset M$ be a bounded domain in a smooth Riemannian manifold $M$ and $F \subset M$ be closed subset with $(n-1)$-Hausdorff measure $\mathcal{H}^{n-1}(F \cap \Omega)=0$. Let $X$ be a vector field of class $C^{1}(\Omega \backslash F) \cap L^{\infty}(\Omega)$ such that $\operatorname{div}(X) \in L^{1}(\Omega)$. Then $X \in \mathcal{W}^{1,1}(\Omega)$ with $\operatorname{Div}(X)=\operatorname{div}(X)$ in $\Omega \backslash F$.

A direct consequence of Theorem 2.23 coupled with Lemma 2.25 is that the Cheng's eigenvalue inequality (3) is valid for arbitrary geodesic balls $B_{M}(r)$ provided the $(n-1)$ Hausdorff measure $\mathcal{H}^{n-1}\left(\operatorname{Cut}(p) \cap B_{N}(p, r)\right)=0$, where $\operatorname{Cut}(p)$ is the cut locus of the center $p$, equivalently that $\operatorname{Cut}(p)$ is made just of focal points. Moreover, the equality in (3) is achieved if and only if $B_{M}(r)$ is isometric to $B_{\mathbb{M}_{\kappa}^{n}}(r)$.

2.6. Cheng's eigenvalue comparison theorem revisited. For notational simplicity, let $\mathbb{M}_{\kappa}^{n}$ denote the space form of curvature $\kappa \in \mathbb{R}$. Using Barta's Theorem 2.21, S. Y. Cheng in [39] proved that

i) if the sectional curvature of $M$ satisfies $K_{M} \leq \kappa$ and $r<\min \{\operatorname{inj}(p), \pi / \sqrt{\kappa}\}$, $(\pi / \sqrt{\kappa}=\infty$ if $\kappa \leq 0)$ then $\lambda_{1}\left(B_{M}(r)\right) \geq \lambda_{1}\left(B_{\mathbb{M}_{\kappa}^{n}}(r)\right)$;

ii) if the Ricci curvature of $M$ satisfies $\operatorname{Ric}_{M} \geq(n-1) \kappa$ then the reverse inequality $\lambda_{1}\left(B_{M}(r)\right) \leq \lambda_{1}\left(B_{\mathbb{M}_{\kappa}^{n}}(r)\right)$ holds for all $r>0$.

Moreover, equality in $i$ ) or $i$ ) holds if and only if the geodesic balls $B_{M}(r)$ and $B_{\mathbb{M}_{\kappa}^{n}}(r)$ are isometric, see [39]. In this section we show that (3) is valid under weaker geometric hypotheses. Let us assume that $B_{M}(r)$ and $B_{\mathbb{M}_{\kappa}^{n}}(r)$ are normal, within the cut locus of their centers and let $(t, \theta) \in(0, r] \times \mathbb{S}^{n-1}$ be geodesic coordinates for $B_{M}(r)$ and $B_{\mathbb{M}_{\kappa}^{n}}(r)$. Let $H_{M}(t, \theta)$ and $H_{\mathbb{M}_{\kappa}^{n}}(t, \theta)=H_{\mathbb{M}_{\kappa}^{n}}(t)$ be the mean curvatures of the distance spheres $\partial B_{M}(t)$ and $\partial B_{\mathbb{M}_{\kappa}^{n}}(t)$ at $(t, \theta)$ with respect to the unit vector field $-\partial / \partial t$. Then the following version of Cheng's Eigenvalue Comparison Theorems is true.

Theorem 2.26 (Bessa-Montenegro-[23]). If $H_{M}(s, \theta) \geq H_{\mathbb{M}_{\kappa}^{n}}(s)$ for all $s \in(0, r]$ and all $\theta \in \mathbb{S}^{n-1}$, then

$$
\lambda_{1}\left(B_{M}(r)\right) \geq \lambda_{1}\left(B_{\mathbb{M}_{\kappa}^{n}}(r)\right) .
$$

If $H_{M}(s, \theta) \leq H_{\mathbb{M}_{\kappa}^{n}}(s)$ for all $s \in(0, r]$ and all $\theta \in \mathbb{S}^{n-1}$, then

$$
\lambda_{1}\left(B_{M}(r)\right) \leq \lambda_{1}\left(B_{\mathbb{M}_{\kappa}^{n}}(r)\right) .
$$

Equality in (29) or in (30) holds if and only if $H_{M}(s, \theta)=H_{\mathbb{M}_{\kappa}^{n}}(s)$ for all $s \in(0, r]$ and for all $\theta \in \mathbb{S}^{n-1}$.

Let $u: B_{\mathbb{M}_{n}^{n}}(r) \rightarrow \mathbb{R}$ be a positive first Dirichlet eigenfunction. It is well known that $u$ is radial function, $u(t, \theta)=u(t), u^{\prime}(t) \leq 0$ and satisfies the following differential equation,

$$
u^{\prime \prime}(s)+(n-1) \frac{C_{\kappa}}{S_{\kappa}}(s) u^{\prime}(s)+\lambda_{1}\left(B_{\mathbb{M}_{\kappa}^{n}}(r)\right) u(s)=0, s \in[0, r]
$$


where $S_{\kappa}(t)$ is defined in (18) and $C_{\kappa}(t) \doteq S_{\kappa}(t)^{\prime}$.

Observe that $u(t, \theta)=u(t)$ also defines a smooth function on $B_{M}(r)$, (called transplanted function), with gradient $\nabla u=u^{\prime} \partial / \partial t$, where $\partial / \partial t$ is the unit vector field normal to the distance spheres $\partial B_{M}(t)$ pointing outward. Therefore

$$
\Delta u=\operatorname{div}\left(u^{\prime} \frac{\partial}{\partial t}\right)=\left\langle\nabla u^{\prime}, \frac{\partial}{\partial t}\right\rangle+u^{\prime} \operatorname{div}\left(\frac{\partial}{\partial t}\right)=u^{\prime \prime}+u^{\prime} \operatorname{Trace}\left(\xi \rightarrow \nabla_{\xi} \frac{\partial}{\partial t}\right) .
$$

Here $\xi \rightarrow \nabla_{\xi} \frac{\partial}{\partial t}$ is the Weingarten map of the distance spheres. Its trace is the mean curvature $H_{M}$. By (31) and (32)) we have at any point $(t, \theta)$ of $B_{M}(r)$ that

$$
\begin{aligned}
-\frac{\Delta u}{u}(t, \theta) & =\left[-\frac{u^{\prime \prime}}{u}-\frac{u^{\prime}}{u} \operatorname{div}\left(\frac{\partial}{\partial t}\right)\right](t, \theta) \\
& =\left[(n-1) \frac{C_{\kappa}}{S_{\kappa}}(t)-H_{M}(t, \theta)\right] \frac{u^{\prime}}{u}(t)+\lambda_{1}\left(B_{\mathbb{M}_{\kappa}^{n}}(r)\right) .
\end{aligned}
$$

Observing that $H_{\mathbb{M}_{\kappa}^{n}}=(n-1) C_{\kappa} / S_{\kappa}$ we have by Barta's Theorem $(2.21)$ that

$$
\sup _{(t, \theta)}\left[\left(H_{\mathbb{M}_{\kappa}^{n}}-H_{M}\right) \frac{u^{\prime}}{u}\right]+\lambda_{1}\left(B_{\mathbb{M}_{\kappa}^{n}}(r)\right)=\sup _{(t, \theta)}\left(-\frac{\Delta u}{u}\right) \geq \lambda_{1}\left(B_{M}(r)\right)
$$

and

$$
\lambda_{1}\left(B_{M}(r)\right) \geq \inf _{(t, \theta)}\left(-\frac{\Delta u}{u}\right)=\inf _{(t, \theta)}\left[\left(H_{\mathbb{M}_{\kappa}^{n}}-H_{M}\right) \frac{u^{\prime}}{u}\right]+\lambda_{1}\left(B_{\mathbb{M}_{\kappa}^{n}}(r)\right) .
$$

Since $u^{\prime} / u \leq 0$ we have that:

- $H_{M} \geq H_{\mathbb{M}_{\kappa}^{n}} \Rightarrow \inf \left[\left(H_{\mathbb{M}_{\kappa}^{n}}-H_{M}\right) \frac{u^{\prime}}{u}\right] \geq 0$ and $\lambda_{1}\left(B_{M}(r)\right) \geq \lambda_{1}\left(B_{\mathbb{M}_{\kappa}^{n}}(r)\right)$.

- $H_{M} \leq H_{\mathbb{M}_{\kappa}^{n}} \Rightarrow \sup \left[\left(H_{\mathbb{M}_{\kappa}^{n}}-H_{M}\right) \frac{u^{\prime}}{u}\right] \leq 0$ and $\lambda_{1}\left(B_{M}(r)\right) \leq \lambda_{1}\left(B_{\mathbb{M}_{\kappa}^{n}}(r)\right)$.

- If $\lambda_{1}\left(B_{M}(r)\right)=\lambda_{1}\left(B_{\mathbb{M}^{n} \kappa}(r)\right)$ we have that $\lambda_{1}\left(B_{M}(r)\right)=\inf _{(t, \theta)}\left(-\frac{\Delta u}{u}\right)$. As observed in Barta's Theorem, the transplanted function $u$ is a positive eigenfunction of $B_{M}(r)$ and from (33) we have that

$$
\left[(n-1) \frac{C_{\kappa}}{S_{\kappa}}-H_{M}\right] \frac{u^{\prime}}{u}(s)=0
$$

for all $s \in[0, r]$. Since $u^{\prime} / u<0$ in $(0, r]$ then $(n-1) \frac{C_{\kappa}}{S_{\kappa}}-H_{M}=0$ in $(0, r]$. By continuity $(n-1) \frac{C_{\kappa}}{S_{\kappa}}-H_{M}=0$ in $[0, r]$.

This finishes the proof of Theorem (2.26). It is clear that using Barta's theorem, Cheng's eigenvalue comparison theorem can be extended to bounded open sets. More precisely, the following result holds.

Theorem 2.27. Let $\Omega \subset M$ be a bounded open subset of a Hadamard n-manifold with sectional curvature $K_{M} \leq \kappa \leq 0$. Then

$$
\lambda^{*}(\Omega) \geq \lambda_{1}\left(B_{\mathbb{M}_{\kappa}^{n}}\left(r_{\Omega}\right)\right),
$$

where $r_{\Omega}$ is the radius of $\Omega$. This is, $r_{\Omega}=\inf _{x \in \Omega} \sup _{y \in \Omega} \operatorname{dist}_{M}(x, y)$.

Theorem 2.26 is just an observation on Cheng's proof and it can be extended to a comparison theorem with model manifolds with no extra effort, see [23, Cor. 4.1] and [65]. However it is important to show that this result above is truly an extension of Cheng's eigenvalue theorem. 
2.7. Examples . If the sectional curvature is bounded above $K_{M} \leq \kappa$, then the mean curvature of the distance spheres $H_{M}(s, \theta) \geq H_{\mathbb{M}_{\kappa}^{n}}(s)$ and if the Ricci curvature is bounded below $\operatorname{Ric}_{M} \geq(n-1) \kappa$ then $H_{M}(s, \theta) \leq H_{\mathbb{M}_{\kappa}^{n}}(s)$. The converse statements are not true in general. We will construct complete model manifolds $\mathbb{M}_{h}^{n}$ with radial sectional curvature $K_{\mathbb{M}_{h}^{n}}^{\mathrm{rad}}>\kappa$ outside a compact set with mean curvatures of the distance spheres satisfying $H_{\mathbb{M}_{h}^{n}}(t, \theta) \geq H_{\mathbb{M}_{n}^{n}}(t, \theta)$ and with radial sectional curvature $K_{\mathbb{M}_{h}^{n}}^{\text {rad }}<\kappa$ outside a compact set with mean curvatures of the distance spheres satisfying $H_{\mathbb{M}_{h}^{n}}(t, \theta) \leq H_{\mathbb{M}_{\kappa}^{n}}(t, \theta)$.

Example 2.28. Let $\mathbb{R}^{n}=[0, \infty) \times \mathbb{S}^{n-1}$ with the metric $d s^{2}=d t^{2}+f^{2}(t) d \theta^{2}, f(0)=0$, $f^{\prime}(0)=1, f^{\prime \prime}(0)=0$. Set $\psi_{\kappa}(t)=\left(-f^{\prime} S_{\kappa}+f S_{\kappa}^{\prime}\right)(t)$, where' means differentiation with respect to $t$. The radial sectional curvature of $\left(\mathbb{R}^{n}, d s^{2}\right)$ is bounded above by $\kappa$ if and only if $\psi_{\kappa}^{\prime}(t) \leq 0$. The mean curvatures of $\partial B_{\mathbb{R}^{n}}(t)$ and $\partial B_{\mathbb{M}_{\kappa}^{n}}(t)$ satisfies $H_{\mathbb{R}^{n}}(t, \theta) \geq H_{\mathbb{M}_{\kappa}^{n}}(t)$ if and only if $\psi_{\kappa}(t) \leq 0$. From $\psi_{\kappa}(t)=\left(-f^{\prime} S_{\kappa}+f S_{\kappa}^{\prime}\right)(t)$ we have that $\psi_{\kappa}(0)=\psi_{\kappa}^{\prime}(0)=0$. Observe that for $t \neq 0$

$$
\frac{\psi_{\kappa}}{S_{\kappa}^{2}}=\frac{-f^{\prime} S_{\kappa}+f S_{\kappa}^{\prime}}{S_{\kappa}^{2}}=-\left(\frac{f}{S_{\kappa}}\right)^{\prime}
$$

Moreover, $\lim _{t \rightarrow 0} \frac{\psi_{\kappa}}{S_{\kappa}^{2}}(t)=0$. Integrating $\left(f / S_{\kappa}\right)^{\prime}$ between $\epsilon$ and $t$ we obtain

$$
\int_{\epsilon}^{t}\left(\frac{f}{S_{\kappa}}\right)^{\prime}(s) d s=\frac{f}{S_{\kappa}}(t)-\frac{f}{S_{\kappa}}(\epsilon)=-\int_{\epsilon}^{t} \frac{\psi_{\kappa}}{S_{\kappa}^{2}}(s) d s
$$

Since $\lim _{\epsilon \rightarrow 0} \frac{f}{S_{\kappa}}(\epsilon)=1$ we have that

$$
f(t)=S_{\kappa}(t)-S_{\kappa}(t) \cdot \int_{0}^{t} \psi_{\kappa}(s) / S_{\kappa}^{2}(s) d s
$$

Let $\psi_{\kappa}:[0, \infty) \rightarrow \mathbb{R}$ be a smooth function satisfying $\psi_{\kappa}(0)=\psi_{\kappa}^{\prime}(0)=0, \psi(t) \leq 0, \psi_{\kappa}^{\prime}(t)>$ 0 for $t>1$ and $\left|\int_{[0, \infty)} \psi_{\kappa}(s) / S_{\kappa}^{2}(s) d s\right|<\infty$. This yields complete metrics $d s^{2}=d t^{2}+$ $f^{2}(t) d \theta$ on $\mathbb{R}^{n}$ with radial sectional curvature $K_{\left(\mathbb{R}^{n}, d s^{2}\right)}>\kappa$ outside the ball $B_{\left(\mathbb{R}^{n}, d s^{2}\right)}(1)$ and so that the distance spheres $\partial B_{\left(\mathbb{R}^{n}, d s^{2}\right)}(t)$ have mean curvature $H_{\left(\mathbb{R}^{n}, d s^{2}\right)}(t, \theta) \geq H_{\mathbb{M}_{\kappa}^{n}}(t, \theta)$. If $\psi(t) \geq 0,\left|\int_{[0, \infty)} \psi_{\kappa}(s) / S_{\kappa}^{2}(s) d s\right|<1$ and such that $\psi_{\kappa}^{\prime}(t)<0$ for $t>1$ we obtain a smooth metric with sectional curvature $K_{\left(\mathbb{R}^{n}, d s^{2}\right)}<\kappa$ outside a compact set with $H_{\left(\mathbb{R}^{n}, d s^{2}\right)}(t, \theta) \leq H_{\mathbb{M}_{\kappa}^{n}}(t, \theta)$.

Remark 2.29. For metrics $d s^{2}=d t^{2}+f^{2}(t) d \theta^{2}$, the equality $H_{\left(\mathbb{R}^{n}, d s^{2}\right)}(t, \theta)=H_{\left(\mathbb{M}_{\kappa}^{n}, \operatorname{can}_{\kappa}\right)}(t)$ for all $t \in(0, r]$ implies that $d s^{2}=d t^{2}+S_{\kappa}^{2} d \theta^{2}$. Since

$$
H_{\left.\mathbb{R}^{n}, d s^{2}\right)}(t)=(n-1)\left(f^{\prime} / f\right)(t)=(n-1)\left(C_{\kappa} / S_{\kappa}\right)(t)=H_{\mathbb{M}_{\kappa}}(t)
$$

implies that $\psi_{\kappa}(t)=0$ for all $t \in[0, r]$ and by (36) $f \equiv S_{\kappa}$.

In a second example, we show that the rigidity in Theorem 2.26 is sharp, constructing a smooth complete metric on $\mathbb{R}^{4}=[0, \infty) \times \mathbb{S}^{3}$ with the following properties.

i. The set $[0, r] \times \mathbb{S}^{3}$ endowed with the metrics $\operatorname{can}_{\kappa}$ or $g_{\kappa}$ are geodesic balls of radius $r$, i.e. $B_{\operatorname{can}_{\kappa}}(r)=\left([0, r] \times \mathbb{S}^{3}, \operatorname{can}_{\kappa}\right)$ and $B_{g_{\kappa}}(r)=\left([0, r] \times \mathbb{S}^{3}, g_{\kappa}\right)$.

ii. $B_{\operatorname{can}_{\kappa}}(r)$ and $B_{g_{\kappa}}(r)$ are not isometric if $\kappa \neq 0$ but have the same first eigenvalue $\lambda_{1}\left(B_{\mathrm{Can}_{\kappa}}(r)\right)=\lambda_{1}\left(B_{g_{\kappa}}(r)\right)$ and the same first eigenfunctions.

iii. The geodesic balls $B_{\operatorname{can}_{\kappa}}(r)$ and $B_{g_{\kappa}}(r)$ and their boundaries $\partial B_{\operatorname{can}_{\kappa}}(r), \partial B_{g_{\kappa}}(r)$ have the same volume $\operatorname{vol}\left(B_{\operatorname{can}_{\kappa}}(r)\right)=\operatorname{vol}\left(B_{g_{\kappa}}(r)\right)$ and $\operatorname{vol}\left(\partial B_{\operatorname{can}_{\kappa}}(r)\right)=\operatorname{vol}\left(\partial B_{g_{\kappa}}(r)\right)$.

iv. The geodesic spheres $\left(\partial B(t), \operatorname{can}_{\kappa}\right)$ and $\left(\partial B(t), g_{\kappa}\right)$ have the same mean curvatures $H_{g_{\kappa}}(t, x)=H_{\operatorname{can}_{\kappa}}(t, x)=C_{\kappa}(t) / S_{\kappa}(t)$ for every $t \in(0, r]$ and $x \in \mathbb{S}^{3}$. 
Example 2.30. Let $\{\partial x, \partial y, \partial z\}$ be a globally defined frame on $\mathbb{S}^{3}$ with $[\partial x, \partial y]=2 \partial x$, $[\partial y, \partial z]=2 \partial x,[\partial z, \partial x]=2 \partial y$ and let $d x$, $d y$ and $d z$ be its dual co-frame. Consider the following metric $d s^{2}=d t^{2}+a^{2}(t) d x^{2}+b^{2}(t) d y^{2}+c^{2}(t) d z^{2}$, where $a, b, c:[0, r] \rightarrow \mathbb{R}$ are smooth functions, with $a(0)=b(0)=c(0)=0$ and $a^{\prime}(0)=b^{\prime}(0)=c^{\prime}(0)=1$ and positive in $(0, r)$. Here $d t$ is the dual co-frame of the radial vector field $\partial t$. Observe that $[\partial t, \partial x]=[\partial t, \partial y]=[\partial t, \partial z]=0$. As mentioned before, taking $a(t)=b(t)=c(t)=S_{\kappa}(t)$ we obtain the standard metric $\mathrm{can}_{\kappa}$ of constant sectional curvature $\kappa$. In our example, we set $a(t)=S_{\kappa}^{2}(t) / t, b(t)=t, c(t)=S_{\kappa}(t)$, where we are assuming that $r<\pi / \sqrt{\kappa}$ if $\kappa>0$. We obtain a smooth metric $g_{\kappa}$ on $[0, r] \times \mathbb{S}^{3}$ that clearly is non-isometric to $\operatorname{can}_{\kappa}$ if $\kappa \neq 0$. For instance, computing the sectional curvatures $K(\partial t, \partial x), K(\partial t, \partial y), K(\partial t, \partial z)$ at the origin we get $K(\partial t, \partial x)=2 \kappa, K(\partial t, \partial y)=0, K(\partial t, \partial z)=\kappa$. Moreover, $[0, r] \times \mathbb{S}^{3}$ is the (closed) geodesic ball of radius $r$ centered at the origin $0=\{0\} \times \mathbb{S}^{3}$ with respect to both metrics $\operatorname{can}_{\kappa}, g_{\kappa}$, since the function $\rho:[0, r] \times \mathbb{S}^{3} \rightarrow \mathbb{R}$ given by $\rho(t, x)=t$ is the distance function to the origin for both metrics. The Laplace operators $\Delta_{g_{\kappa}}, \Delta_{\operatorname{can}_{\kappa}}$ of $g_{\kappa}$ and $\operatorname{can}_{\kappa}$ written in these coordinates, setting $\partial t=\partial / \partial t, \partial x=\partial / \partial x, \partial y=\partial / \partial y, \partial z=\partial / \partial z$ for a classic notation, are given by

$$
\begin{aligned}
\Delta_{g_{\kappa}} & =\frac{\partial^{2}}{\partial t^{2}}+(n-1) \frac{C_{\kappa}}{S_{\kappa}} \frac{\partial}{\partial t}+\frac{t^{2}}{S_{\kappa}^{4}} \frac{\partial^{2}}{\partial x^{2}}+\frac{1}{t^{2}} \frac{\partial^{2}}{\partial y^{2}}+\frac{1}{S_{\kappa}^{2}} \frac{\partial^{2}}{\partial z^{2}} \\
\Delta_{\operatorname{can}_{\kappa}} & =\frac{\partial^{2}}{\partial t^{2}}+(n-1) \frac{C_{\kappa}}{S_{\kappa}} \frac{\partial}{\partial t}+\frac{1}{S_{\kappa}^{2}} \frac{\partial^{2}}{\partial x^{2}}+\frac{1}{S_{\kappa}^{2}} \frac{\partial^{2}}{\partial y^{2}}+\frac{1}{S_{\kappa}^{2}} \frac{\partial^{2}}{\partial z^{2}} .
\end{aligned}
$$

Let $u: B_{\operatorname{can}_{\kappa}}(r) \rightarrow \mathbb{R}$ be a positive first eigenfunction. This means that $u$ satisfies the following boundary value problem

$$
\left\{\begin{array}{rll}
\Delta_{\operatorname{can}_{\kappa}} u+\lambda_{1}\left(B_{\operatorname{can}_{\kappa}}(r)\right) u & =0 & \text { in } B_{\operatorname{can}_{\kappa}}(r) \\
u & =0 & \text { on } \partial B_{\operatorname{can}_{\kappa}}(r) .
\end{array}\right.
$$

It is well known, see [33], that $u$ is radial, i.e. $u(t, x)=u(t)$. From (37) we see that the Laplace operators $\Delta_{g_{\kappa}}$ and $\Delta_{\mathrm{can}_{\kappa}}$ coincide on the set of smooth radial functions defined on $[0, r] \times \mathbb{S}^{3}$. Thus we have $\Delta_{g_{\kappa}} u=\Delta_{\operatorname{can}_{\kappa}} u$. This implies that $u$ satisfies the following boundary value problem

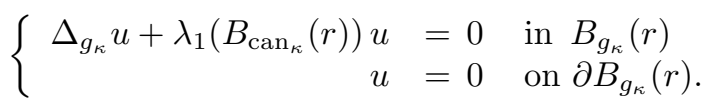

This shows that $u$ is an eigenfunction of $B_{g_{\kappa}}(r)$. Since $u>0$, it is a first eigenfunction and $\lambda_{1}\left(B_{\operatorname{can}_{\kappa}}(r)\right)=\lambda_{1}\left(B_{g_{\kappa}}(r)\right)$ is the first eigenvalue. Moreover, the mean curvatures of the distance spheres $\partial B_{g_{\kappa}}(t), \partial B_{\operatorname{can}_{\kappa}}(t)$ are just $\Delta_{g_{\kappa}} \rho(t, x)=\Delta_{\operatorname{can}_{\kappa}} \rho(t, x)=\left(C_{\kappa} / S_{\kappa}\right)(t)$.

2.8. Fundamental tone estimates on minimal submanifolds. One of the important applications of Barta's Theorem is the fundamental tones estimates of minimal submanifolds of the space forms. The first estimate known is due to S. Y. Cheng, P. Li and S. T. Yau in [40], where they applied Barta's Theorem to prove the following estimates for compact subsets of minimal submanifolds of space forms.

Theorem 2.31 (Cheng-Li-Yau). Let $\varphi: M \rightarrow \mathbb{N}_{\kappa}^{n}$ be an immersed m-dimensional minimal submanifold of the $n$-dimensional space form of constant sectional curvature $\kappa$, and let $D \subset M$ be a $C^{2}$ compact domain. Let $r=\inf _{p \in D} \sup _{z \in D} \operatorname{dist}_{\mathbb{N}_{n}^{n}}(p, z)>0$ be the outer radius of $D$. If $\kappa>0$ suppose that $r \leq \pi / 2 \sqrt{\kappa}$. Then

$$
\lambda_{1}(D) \geq \lambda_{1}\left(B_{\mathbb{N}_{\kappa}^{m}}(r)\right) .
$$

Equality in (40) holds iff $M$ is totally geodesic in $\mathbb{N}_{\kappa}^{n}$ and $D=B_{\mathbb{N}_{\kappa}^{m}}(r)$. 
Let us consider $\varphi: M \rightarrow N$ a minimal immersion of a complete Riemannian $m$-manifold $M$ into a Riemannian $n$-manifold $N$. Let $B_{N}(r)$ be a geodesic ball with radius $r$ and center at a point $p$. Assume that $\mathcal{H}^{n-1}\left(\operatorname{Cut}(p) \cap B_{N}(r)\right)=0$ and let $\kappa=\sup _{B_{N}(r)} K^{\mathrm{rad}}$ the supremum of the radial sectional curvatures along the geodesics issuing from $p$ and consider $\left.\Omega \subset \varphi^{-1}\left(B_{N}(r)\right)\right)$ a connected component. In the next result we obtain lower estimates for the fundamental $\lambda^{*}(\Omega)$ tone of $\Omega$ and can be seen as an extension of ChengLi-Yau Theorem.

Theorem 2.32 (Bessa-Montenegro-[22]). In the setting above, let us assume further that $r<\pi / 2 \sqrt{\kappa}$ if $\kappa>0$. Then we have

$$
\lambda^{*}(\Omega) \geq \lambda_{1}\left(B_{\mathbb{N}_{\kappa}^{m}}(r)\right),
$$

where $B_{\mathbb{N}_{\kappa}^{m}}(r)$ is the geodesic ball with radius $r$ in the simply connected space form $\mathbb{N}_{\kappa}^{m}$ of constant sectional curvature $\kappa$. If $\Omega$ is bounded, then equality in (41) holds iff $\Omega=B_{\mathbb{N}_{\kappa}^{m}}(r)$.

Corollary 2.33. Let $\varphi: M \rightarrow N$ be a minimal immersion of a complete Riemannian mmanifold $M$ into a Riemannian n-manifold $N$. Assume that $\mathcal{H}^{n-1}\left(\operatorname{Cut}(p) \cap B_{N}(r)\right)=0$ and let $\kappa=\sup _{B_{N}(r)} K^{\mathrm{rad}}$ the supremum of the radial sectional curvatures along the geodesics issuing from $p$. If $\varphi(M) \subset B_{N}(r)$, then

$$
\lambda^{*}(\Omega) \geq \lambda_{1}\left(B_{\mathbb{N}_{\kappa}^{m}}(r)\right) .
$$

In the last decade there has been a great development in theory of extrinsically bounded minimal surfaces of $\mathbb{R}^{3}$ with the discovery of a wealth of examples of complete bounded minimal surfaces, see [2], [3], [4], [63], [92], [93], [99], [100], [101], [102], [132]. In those examples, we have that $\Omega=M$ and $\lambda^{*}(M) \geq \lambda_{1}\left(B_{\mathbb{R}^{2}}(r)\right) \approx 5.78 / r^{2}$.

Proof. Let $v: B_{\mathbb{N}_{\kappa}^{m}}(r) \rightarrow \mathbb{R}$ be a positive first Dirichlet eigenfunction of $B_{\mathbb{N}_{\kappa}^{m}}(r)$. It is known that $v$ is radial with $v^{\prime}(t) \leq 0$ and $v^{\prime}(t)=0$ iff $t=0$. We can normalize $v$ such that $v(0)=1$. The differential equation $\Delta_{\mathbb{N}_{\kappa}^{m}} v(t)+\lambda_{1}\left(B_{\mathbb{N}_{\kappa}^{m}}(r)\right) v(t)=0$ is expressed in geodesic coordinates by

$$
v^{\prime \prime}(t)+(m-1) \frac{C_{\kappa}(t)}{S_{\kappa}(t)} v^{\prime}(t)+\lambda_{1}\left(B_{\mathbb{N}_{\kappa}^{m}}(r)\right) v(t)=0, \forall t \in[0, r] .
$$

For each $\xi \in T_{p} N,|\xi|=1, d(\xi)>0$ is the largest real number (possibly $\infty$ ) such that geodesic $\gamma_{\xi}(t)=\exp _{p}(t \xi)$ minimizes the distance from $\gamma_{\xi}(0)=p$ to $\gamma_{\xi}(t), 0 \leq t \leq d(\xi)$. We have that $B_{N}(p, r) \backslash \operatorname{Cut}(p)=\exp _{p}\left(\left\{t \xi \in T_{p} N: 0 \leq t<\min \{r, d(\xi)\},|\xi|=1\right\}\right)$. Define $u: B_{N}(p, r) \rightarrow \mathbb{R}$ by $u\left(\exp _{p}(t \xi)\right)=v(t)$ if $t<\min \{r, d(\xi)\}$ and $u(r \xi)=u(d(\xi) \xi)=0$. Define $\psi: \Omega \rightarrow \mathbb{R}$ defined by $\psi=u \circ \varphi$. The vector field $X=-\nabla \log \psi$ identified with $d \varphi(X)$ is not smooth at $F=\varphi^{-1}\left(\operatorname{Cut}_{N}(p)\right)$. By hypothesis $\mathcal{H}^{m-1}(\Omega \cap F)=0$ and it can be shown that the vector field $X \in C^{1}(\Omega \backslash F) \cap L^{\infty}(\Omega)$ and $\operatorname{div} X \in L^{1}(\Omega)$ thus $X \in \mathcal{W}^{1,1}(\Omega)$, see Lemma 2.25 and by Theorem (2.23) and Remark (2.24) we have that

$$
\lambda^{*}(\Omega) \geq \inf _{\Omega \backslash F}\left[\operatorname{Div} X-|X|^{2}\right]=\inf _{\Omega \backslash F}\left[\operatorname{div} X-|X|^{2}\right]=\inf _{\Omega \backslash F}[-\Delta \psi / \psi] .
$$

Where $\Delta \psi$ is given by the following formula, [82],

$$
\begin{aligned}
\Delta \psi(x) & =\sum_{i=1}^{m} \operatorname{Hess} u(\varphi(x))\left(e_{i}, e_{i}\right)+\langle\nabla u, \vec{H}\rangle \\
& =\sum_{i=1}^{m} \operatorname{Hess} u(\varphi(x))\left(e_{i}, e_{i}\right),
\end{aligned}
$$

where $\varphi(x)=\exp _{p}(t \xi), \vec{H}=0$ is the mean curvature vector of $\Omega$ at $\varphi(x)$ and $\left\{e_{1}, \ldots, e_{m}\right\}$ is an orthonormal basis for $T_{\varphi(x)} \Omega$. Choose this basis such that $e_{2}, \ldots, e_{m}$ are tangent 
to the distance sphere $\partial B_{N}(p, t) \subset N$ and $e_{1}=\cos (\beta(x)) \partial / \partial t+\sin (\beta(x)) \partial / \partial \theta$, where $\partial / \partial \theta \in\left[\left[e_{2}, \ldots e_{m}\right]\right],|\partial / \partial \theta|=1$. From (44) we have for $\varphi(x) \in \Omega \backslash F$ that

$$
\begin{aligned}
\Delta \psi(x)= & \sum_{i=1}^{m} \operatorname{Hess} u(\varphi(x))\left(e_{i}, e_{i}\right) \\
= & v^{\prime \prime}(t)\left(1-\sin ^{2} \beta(x)\right)+v^{\prime}(t) \sin ^{2} \beta(x) \operatorname{Hess}(t)(\partial / \partial \theta, \partial / \partial \theta) \\
& +v^{\prime}(t) \sum_{i=2}^{m} \operatorname{Hess}(t)\left(e_{i}, e_{i}\right)
\end{aligned}
$$

where $t=\operatorname{dist}_{N}(p, x)$. Add and subtract $\left(\frac{C_{\kappa}}{S_{\kappa}}\right)(t) v^{\prime}(t) \sin ^{2} \beta(x)$ and $(m-1)\left(\frac{C_{\kappa}}{S_{\kappa}}\right)(t) v^{\prime}(t)$ in (45) to obtain

$$
\begin{aligned}
\Delta \psi(x) & =v^{\prime \prime}(t)+(m-1) \frac{C_{\kappa}}{S_{\kappa}}(t) v^{\prime}(t) \\
& +\left(\operatorname{Hess}(t)(\partial / \partial \theta, \partial / \partial \theta)-\frac{C_{\kappa}(t)}{S_{\kappa}(t)}\right) v^{\prime}(t) \sin ^{2} \beta(x) \\
& +\sum_{i=2}^{m}\left[\operatorname{Hess}(t)\left(e_{i}, e_{i}\right)-\frac{C_{\kappa}}{S_{\kappa}}(t)\right] v^{\prime}(t)+\left(\frac{C_{\kappa}(t)}{S_{\kappa}(t)} v^{\prime}(t)-v^{\prime \prime}(t)\right) \sin ^{2} \beta(x)
\end{aligned}
$$

Substituting (43) into (46) we have that

$$
\begin{aligned}
-\frac{\Delta \psi}{\psi}(x) & =\lambda_{1}\left(B_{\mathbb{N}_{\kappa}^{m}}(r)\right) \\
& -\left(\operatorname{Hess}(t)(\partial / \partial \theta, \partial / \partial \theta)-\frac{C_{\kappa}(t)}{S_{\kappa}(t)}\right) \frac{v^{\prime}(t)}{v(t)} \sin ^{2} \beta(x) \\
& -\sum_{i=2}^{m}\left[\operatorname{Hess}(t)\left(e_{i}, e_{i}\right)-\frac{C_{\kappa}}{S_{\kappa}}(t)\right] \frac{v^{\prime}(t)}{v(t)} \\
& -\frac{1}{v(t)}\left(\frac{C_{\kappa}(t)}{S_{\kappa}(t)} v^{\prime}(t)-v^{\prime \prime}(t)\right) \sin ^{2} \beta(x) .
\end{aligned}
$$

Since the radial curvature $K(x)(\partial t, v) \leq \kappa$ for all $x \in B_{N}(p, r) \backslash \operatorname{Cut}(p)$ and all $v \perp$ $\partial t$ with $|v| \leq 1$ we have by the Hessian Comparison Theorem (see [125]) we have that Hess $(t(x))(v, v) \geq\left(C_{\kappa} / S_{\kappa}\right)(t)$ for all $v \perp \partial t, t(x)=t, x=\exp _{p}(t \xi)$. But $v^{\prime}(t) \leq 0$ then we have that the second and third terms of (47) are non-negative. If the fourth term of (47) is non-negative then we would have that

$$
-\frac{\Delta \psi}{\psi}(x) \geq \lambda_{1}\left(B_{\mathbb{N}_{\kappa}^{m}}(r)\right) .
$$

By Theorem (2.23) we have that

$$
\lambda^{*}(\Omega) \geq \inf \left(-\frac{\Delta \psi}{\psi}\right) \geq \lambda_{1}\left(B_{\mathbb{N}_{\kappa}^{m}}(r)\right)
$$

This proves (41). We can see that $-\left(\frac{C_{\kappa}(t)}{S_{\kappa}(t)} \frac{v^{\prime}(t)}{v(t)}-\frac{v^{\prime \prime}(t)}{v(t)}\right) \sin ^{2} \beta(x) \geq 0$ is equivalent to

$$
m \frac{C_{\kappa}(t)}{S_{\kappa}(t)} v^{\prime}(t)+\lambda_{1}\left(B_{\mathbb{N}_{\kappa}^{m}}(r)\right) v(t) \leq 0, t \in(0, r)
$$


To prove (49) we will assume without loss of generality that $\kappa=-1,0,1$. Let us consider first the case $c=0$ that presents the idea of the proof. The other two remaining cases $(\kappa=-1$ and $\kappa=1)$ we are going to treat (quickly) with the same idea. When $\kappa=0$ the inequality (49) becomes

$$
\frac{m}{t} v^{\prime}(t)+\lambda_{1} v(t) \leq 0, t \in(0, r),
$$

where $\lambda_{1}:=\lambda_{1}\left(B_{\mathbb{N}_{\kappa}^{m}}(r)\right)$. Let $\mu(t):=\exp \left\{-\frac{\lambda_{1} t^{2}}{2 m}\right\}$. The functions $v$ and $\mu$ satisfy the following identities,

$$
\begin{aligned}
& \left(t^{m-1} v^{\prime}(t)\right)^{\prime}+\lambda_{1} t^{m-1} v(t)=0 \\
& \left(t^{m-1} \mu^{\prime}(t)\right)^{\prime}+\lambda_{1} t^{m-1}\left(1-\frac{\lambda_{1} t^{2}}{m^{2}}\right) \mu(t)=0 .
\end{aligned}
$$

In (51) we multiply the first identity by $\mu$ and the second by $-v$ adding them and integrating from 0 to $t$ the resulting identity we obtain,

$$
t^{m-1} v^{\prime}(t) \mu(t)-t^{m-1} v(t) \mu^{\prime}(t)=-\frac{\lambda_{1}^{2}}{m^{2}} \int_{0}^{t} \mu(t) v(t)<0, \quad \forall t \in(0, r) .
$$

Then $\mu(t) v^{\prime}(t)<\mu^{\prime}(t) v(t)$ and this proves (50).

Assume that now that $\kappa=-1$. Inequality (49) becomes

$$
m \frac{C_{-1}(t)}{S_{-1}(t)} v^{\prime}(t)+\lambda_{1} v(t)<0 .
$$

Set $\mu(t):=C_{-1}(t)^{-\lambda_{1} / m}$. The functions $v$ and $\mu$ satisfy the the following identities

$$
\begin{aligned}
& \left(S_{-1}^{m-1} v^{\prime}\right)^{\prime}+\lambda_{1} S_{-1}^{m-1} v=0, \\
& \left(S_{-1}^{m-1} \mu^{\prime}\right)^{\prime}+\lambda_{1} S_{-1}^{m-1}\left(\frac{m-1}{m}+\frac{1}{m C_{-1}^{2}}-\frac{\lambda_{1}}{m^{2}} \frac{S_{-1}^{2}}{C_{-1}^{2}}\right) \mu=0 .
\end{aligned}
$$

In (53) we multiply the first identity by $\mu$ and the second by $-v$ adding them and integrating from 0 to $t$ the resulting identity we obtain

$$
S_{-1}^{m-1}\left(v^{\prime} \mu-\mu^{\prime} v\right)(t)+\int_{0}^{t} \lambda_{1} S_{-1}^{m-1}\left[\frac{1}{m}-\frac{1}{m C_{-1}^{2}}+\frac{\lambda_{1}}{m^{2}} \frac{S_{-1}^{2}}{C_{-1}^{2}}\right] \mu v=0 .
$$

The term $S_{-1}^{m-1}\left[\frac{1}{m}-\frac{1}{m C_{-1}^{2}}+\frac{\lambda_{1}}{m^{2}} \frac{S_{-1}^{2}}{C_{-1}^{2}}\right] \mu v$ is positive (one can easily check) therefore we have that $\left(v^{\prime} \mu-\mu^{\prime} v\right)(t)<0$ for all $t \in(0, r)$. This proves (52).

For $\kappa=1$ the inequality (49) becomes the following inequality

$$
m \frac{C_{1}}{S_{1}} v^{\prime}(t)+\lambda_{1} v(t)<0, \quad 0<t<\pi / 2 .
$$

Set $\mu(t):=C_{1}(t)^{-\lambda_{1} / m}, 0<t<\pi / 2$. The functions $v$ and $\mu$ satisfy the the following identities

$$
\begin{aligned}
& \left(S_{1}^{m-1} v^{\prime}\right)^{\prime}+\lambda_{1} S_{1}^{m-1} v=0 \\
& \left(S_{1}^{m-1} \mu^{\prime}\right)^{\prime}-\lambda_{1} S_{1}^{m-1}\left(\frac{m-1}{m}+\frac{1}{m C_{1}^{2}}+\frac{\lambda_{1}}{m^{2}} \frac{S_{1}^{2}}{C_{1}^{2}}\right) \mu=0 .
\end{aligned}
$$


In (55) we multiply the first identity by $\mu$ and the second by $-v$ adding them and integrating from 0 to $t$ the resulting identity we obtain

$$
S_{1}^{m-1}\left(v^{\prime} \mu-\mu^{\prime} v\right)(t)+\int_{0}^{t} \lambda_{1} S_{1}^{m-1}\left[2-\frac{1}{m}+\frac{1}{m C_{1}^{2}}+\frac{\lambda_{1}}{m^{2}} \frac{S_{1}^{2}}{C_{1}^{2}}\right] \mu v=0 .
$$

The term $S_{1}^{m-1}\left[2-\frac{1}{m}+\frac{1}{m C_{1}^{2}}+\frac{\lambda_{1}}{m^{2}} \frac{S_{1}^{2}}{C_{1}^{2}}\right] \mu v$ is positive thus $\left(v^{\prime} \mu-\mu^{\prime} v\right)(t)<0$ for all $t \in(0, r)$. This proves (54) and thus the fourth term in (47) is non-negative. If $\Omega$ is bounded and suppose that $\lambda^{*}(\Omega)=\lambda_{1}\left(B_{\mathbb{N}_{\kappa}^{m}}(r)\right)$. Then first we have that $\varphi(\partial \Omega) \subset \partial B_{N}(p, r)$ and second that the function $\psi=u \circ \varphi \in C^{2}(\Omega)$ by Proposition (2.21) is an eigenfunction of $\Omega$ and thus $\lambda^{*}(\Omega)=-\Delta \psi / \psi$. From (47) we have that

$$
\begin{aligned}
\left(\operatorname{Hess}(t)\left(\frac{\partial}{\partial \theta}, \frac{\partial}{\partial \theta}\right)-\frac{C_{\kappa}(t)}{S_{\kappa}(t)}\right) \frac{v^{\prime}(t)}{v(t)} \sin ^{2} \beta(x) & =0 \\
\sum_{i=2}^{m}\left[\operatorname{Hess}(t)\left(e_{i}, e_{i}\right)-\frac{C_{\kappa}}{S_{\kappa}}(t)\right] \frac{v^{\prime}(t)}{v(t)} & =0 \\
\frac{1}{v(t)}\left(\frac{C_{c}(t)}{S_{c}(t)} v^{\prime}(t)-v^{\prime \prime}(t)\right) \sin ^{2} \beta(x) & =0
\end{aligned}
$$

for all $t$ such that $\varphi(x)=\exp _{p}(t \xi) \in \Omega$. This implies $\sin ^{2} \beta(x)=0$ for all $x \in \Omega$ and we have that $e_{1}(\varphi(x))=\partial / \partial t$. Integrating the vector field $\partial / \partial t$ we have a minimal geodesic (in $N \cap \varphi(\Omega)$ ) joining $\varphi(x)$ to the center $p$. This imply that $\Omega$ is the geodesic ball in $M$ centered at $\varphi^{-1}(p)$ with radius $r$ i.e. $\Omega=B_{M}\left(\varphi^{-1}(p), r\right)$. Since $\psi$ is an eigenfunction with the same eigenvalue $\lambda_{1}\left(B_{\mathbb{N}^{m}}(c)(r)\right)$ we have that

$$
\Delta_{M} v(t)=\Delta_{\mathbb{N}^{m}(c)} v(t), t=\operatorname{dist}_{N}(p, \varphi(q)), \forall q \in \Omega .
$$

Rewriting this identity (56) in geodesic coordinates we have that

$$
\frac{\sqrt{g(t, \xi)}^{\prime}}{\sqrt{g(t, \xi)}}(t, \theta) v^{\prime}(t)+v^{\prime \prime}(t)=(m-1) \frac{C_{c}(t)}{S_{c}(t)} v^{\prime}(t)+v^{\prime \prime}(t) .
$$

This imply that by Bishop Theorem $\Omega=B_{M}\left(\varphi^{-1}(p)\right.$ and $B_{\mathbb{N}^{m}(c)}(r)$ are isometric.

\section{ON DiscRETE AND ESSENTIAL SPECTRUM OF MANIFOLDS}

The above section was devoted to produce estimates for the bottom of $\sigma(M)$. In the next sections, we investigate criteria to ensure that $\sigma(M)$ is discrete (that is, a divergent sequence of eigenvalues, each one with finite multiplicity) or not. We begin with a brief overview of facts from spectral theory that will be used in the sequel.

Let $K \subset M$ be a compact set of the same dimension as $M$. The Laplace-Beltrami operator $\Delta$ of $M$ acting on the space $C_{0}^{\infty}(M \backslash K)$ of smooth compactly supported functions of $M \backslash K$ has a self-adjoint extension, denoted by $\Delta^{\prime}$. The Decomposition Principle [53] says that $\sigma_{\text {ess }}(M)=\sigma_{\text {ess }}(M \backslash K)$. On the other hand,

$$
0 \leq \lambda^{*}(M \backslash K)=\inf \sigma(M \backslash K) \leq \inf \sigma_{\text {ess }}(M \backslash K)=\sigma_{\text {ess }}(M),
$$

thus $\mu=\sup \left\{\lambda^{*}(M \backslash K), K \subset M\right.$ compact $\} \leq \inf \sigma_{\text {ess }}(M)$. We are going to show that $\inf \sigma_{\text {ess }}(M) \leq \mu$. To that we may suppose that $\mu<\infty$, otherwise there is nothing to prove. Let $K_{1} \subset K_{2} \subset \cdots$ be a sequence of compact sets with $M=\cup_{i=1}^{\infty} K_{i}$. We have that

$$
\lambda^{*}(M) \leq \lambda^{*}\left(M \backslash K_{1}\right) \leq \lambda^{*}\left(M \backslash K_{2}\right) \leq \cdots \rightarrow \mu .
$$


Given $\varepsilon>0$, there exists $f_{1} \in C_{0}^{\infty}\left(M \backslash K_{1}\right)$ with $\left\|f_{1}\right\|_{L^{2}}=1$ and

$$
\int_{M}\left|\operatorname{grad} f_{1}\right|^{2} \leq \lambda^{*}\left(M \backslash K_{1}\right)+\varepsilon<\mu+\varepsilon .
$$

This is $\left\langle(-\Delta-\mu-\varepsilon) f_{1}, f_{1}\right\rangle_{L^{2}}<0$. We can suppose that $\operatorname{supp}\left(f_{1}\right) \subset\left(K_{2} \backslash K_{1}\right)$. There exists $f_{2} \in C_{0}^{\infty}\left(M \backslash K_{2}\right)$ with $\left\|f_{2}\right\|_{L^{2}}=1$ and

$$
\int_{M}\left|\operatorname{grad} f_{2}\right|^{2} \leq \lambda^{*}\left(M \backslash K_{2}\right)+\varepsilon<\mu+\varepsilon .
$$

This is equivalent to $\left\langle(-\Delta-\mu-\varepsilon) f_{2}, f_{2}\right\rangle_{L^{2}}<0$. Since $\operatorname{supp}\left(f_{1}\right) \cap \operatorname{supp}\left(f_{2}\right)=\emptyset$ we have $\int_{M} f_{1} f_{2}=0$. This way, we obtain an orthonormal sequence $\left\{f_{k}\right\} \subset C_{0}^{\infty}(M)$ such that $\left\langle(-\Delta-\mu-\varepsilon) f_{k}, f_{k}\right\rangle_{L^{2}}<0$. By Lemma 3.2 below we have that $(-\infty, \mu] \cap \sigma_{\text {ess }}(M) \neq \emptyset$ and inf $\sigma_{\text {ess }}(M) \leq \mu$. This proves the following proposition, known as Persson's formula.

Proposition 3.1. [114] The infimum of the essential spectrum is characterized by

$$
\inf \sigma_{\mathrm{ess}}(M)=\sup \left\{\lambda^{*}(M \backslash K): K \text { compact subset of } M\right\} \text {. }
$$

In particular, $\sigma_{\mathrm{ess}}(M)$ is empty if and only if given any compact exhaustion $K_{1} \subset K_{2} \subset$ $\cdots \subset K_{n} \subset \ldots$ of $M$, the limit $\lim _{n \rightarrow \infty} \lambda^{*}\left(M \backslash K_{n}\right)$ is infinite.

Let $H$ be a Hilbert space and $A: \mathcal{D} \subset H \rightarrow H$ be a densely defined self-adjoint operator. Given $\lambda \in \mathbb{R}$, we write $A \geq \lambda$ if $\langle A x, x\rangle \geq \lambda\|x\|^{2}$ for all $x \in \mathcal{D}$. By the Spectral Theorem for (unbounded) self-adjoint operators, we have that $A \geq \lambda$ iff $\sigma(A) \subset[\lambda,+\infty)$. Let us write $A>-\infty$ if there exists $\lambda_{*} \in \mathbb{R}$ such that $A \geq \lambda_{*}$.

Lemma 3.2. Let $A: \mathcal{D} \subset H \rightarrow H$ be a self-adjoint operator with $A>-\infty$, and let $\lambda \in \mathbb{R}$ be fixed. Assume that for all $\varepsilon>0$ there exists an infinite dimensional subspace $G_{\varepsilon} \subset \mathcal{D}$ such that $\langle A x, x\rangle<(\lambda+\varepsilon)\|x\|^{2}$ for all $x \in G_{\varepsilon}$. Then,

$$
\sigma_{\mathrm{ess}}(A) \cap(-\infty, \lambda] \neq \emptyset \text {. }
$$

This lemma is well known, see [52] but for sake of completeness we present here its proof. First we will show that $\sigma(A) \cap(-\infty, \lambda]=\sigma(A) \cap\left[\lambda_{*}, \lambda\right] \neq \emptyset$. Take $\varepsilon_{k}=1 / k, k \geq 1$. By our hypothesis there exists $x_{k} \neq 0$ such that $\left\langle A x_{k}, x_{k}\right\rangle<(\lambda+1 / k)\left\|x_{k}\right\|^{2}$, and thus $\sigma(A) \cap\left[\lambda_{*}, \lambda+1 / k\right] \neq \emptyset$ for all $k \geq 1$. Since $\sigma(A)$ is closed, it follows $\sigma(A) \cap(-\infty, \lambda] \neq \emptyset$. We may suppose that $\sigma(A) \cap(-\infty, \lambda] \not \subset \sigma_{\text {ess }}(A)$, otherwise there is nothing to prove. Thus

$$
\left(\sigma(A) \backslash \sigma_{\mathrm{ess}}(A)\right) \cap(-\infty, \lambda]=\left\{\lambda_{1}, \ldots, \lambda_{n}\right\}
$$

is a finite set of eigenvalues of $A$ of finite multiplicity. Denote by $H_{i} \subset \mathcal{D}$ the $\lambda_{i}$-eigenspace of $A, i=1, \ldots, n$, and set $X=\bigoplus_{i} H_{i} \subset \mathcal{D}$. This is clearly an invariant subspace of $A$. Since $X$ has finite dimension, then $\mathcal{D}=X \oplus X_{1}$ where $X_{1}=X^{\perp} \cap \mathcal{D}$ is also invariant by $A$. Denote by $A_{1}$ the restriction of $A$ to the Hilbert space $X_{1}$ which is still self-adjoint. Clearly, $\sigma\left(A_{1}\right)=\sigma(A) \backslash\left\{\lambda_{1}, \ldots, \lambda_{n}\right\}$ and $\sigma_{\text {ess }}\left(A_{1}\right)=\sigma_{\text {ess }}(A)$. In particular, we have $\sigma\left(A_{1}\right) \cap(-\infty, \lambda] \subset \sigma_{\text {ess }}\left(A_{1}\right)$. Using the infinite dimensionality of the space $G_{\varepsilon}$, it is now easy to see that the assumptions of our lemma hold for the operator $A_{1}$, and the first part of the proof applies to obtain $\sigma_{\text {ess }}(A) \cap(-\infty, \lambda]=\sigma_{\text {ess }}\left(A_{1}\right) \cap(-\infty, \lambda] \neq \emptyset$.

\section{Riemannian SUbmersions With DiscRete SPECTRUM}

Given manifolds $M$ and $N$, a smooth surjective map $\pi: M \rightarrow N$ is a submersion if the differential $\mathrm{d} \pi(q)$ has maximal rank for every $q \in M$. If $\pi: M \rightarrow N$ is a submersion, then for all $p \in N$ the inverse image $\mathcal{F}_{p}=\pi^{-1}(p)$ is a smooth embedded submanifold of $M$, that will be called the fiber at $p$. If $M$ and $N$ are Riemannian manifolds, then a submersion $\pi: M \rightarrow N$ is called a Riemannian submersion if for all $p \in N$ and all $q \in \mathcal{F}_{p}$, 
the restriction of $\mathrm{d} \pi(q)$ to the orthogonal subspace $T_{q} \mathcal{F}_{p}^{\perp}$ is an isometry onto $T_{p} M$, see. $[68,109,110]$.

An important class of examples are Riemannian homogeneous spaces $G / K$, where $G$ is a Lie group endowed with a bi-invariant Riemannian metric and $K$ is a closed subgroup of $G$, see [109] for details. The projection $G \rightarrow G / K$ is a Riemannian submersions with totally geodesic fibers, and with fibers diffeomorphic to $K$. Another class of examples can be described as the total space of Riemannian submersions with minimal fibers are the homogeneous Riemannian 3-manifolds with isometry group of dimension four, see [126]. This class includes the special linear group $\operatorname{SL}(2, \mathbb{R})$ endowed with a family of left invariant metrics indexed by $\kappa$ and $\tau$ (curvature of the fibers and torsion), which is the total space of Riemannian submersions with base given by the hyperbolic spaces, and fibers diffeomorphic to $\mathbb{S}^{1}$.

In this section, we consider Riemannian submersions $\pi: M \rightarrow N$ and we prove some spectral estimates relating the (essential) spectrum of $M$ and $N$. When $M$ (and thus also $N$ ) is compact, estimates on the eigenvalues of the Laplacian of $M$ have been studied in [28], under the assumption that the mean curvature vector of the fibers is basic, i.e., $\pi$-related to some vector field on the basis. We will consider here the non compact case, assuming initially that the fibers are minimal. Given a Riemannian submersion $\pi: M \rightarrow N$ with compact minimal fibers, we prove that

$$
\sigma_{\text {ess }}(M)=\emptyset \quad \Longleftrightarrow \quad \sigma_{\text {ess }}(N)=\emptyset,
$$

see Theorem 4.1. This result coincides with Baider's result, see [8], when $M=X \times Y$ is a product manifold, $Y$ is compact, $N=X$ and $\pi: X \times Y \rightarrow X$ is the projection on the first factor. Our main result is the following theorem.

Theorem 4.1 (Bessa-Montenegro-Piccione-[25]). Let $\pi: M \rightarrow N$ be a Riemannian submersion with compact minimal fibers. Then

i. $\sigma_{\text {ess }}(N) \subset \sigma_{\text {ess }}(M), \sigma_{p}(N) \subset \sigma_{p}(M)$, thus $\sigma(N) \subset \sigma(M)$.

ii. $\inf \sigma_{\mathrm{ess}}(N)=\inf \sigma_{\mathrm{ess}}(M)$. Therefore, $M$ is discrete if and only if $N$ is discrete.

Remark 4.2. For the inequality $\inf \sigma_{\mathrm{ess}}(M) \leq \inf \sigma_{\mathrm{ess}}(N)$ we need only the compactness of the fibers with uniformly bounded volume, meaning that $0<c^{2} \leq \operatorname{vol}\left(\mathcal{F}_{p}\right) \leq C^{2}$ for all $p \in N$, see Lemma 4.8. The example of [8] shows that the assumption of minimality of the fibers is necessary in Theorem 4.1. In fact, one has examples of Riemannian submersions having compact fibers with discrete base and non discrete total space, or with discrete total space but not discrete base, see Example 2.7.

4.1. Preliminaries. Given manifolds $M$ and $N$, a smooth surjective map $\pi: M \rightarrow N$ is a submersion if the differential $\mathrm{d} \pi(q)$ has maximal rank for every $q \in M$. If $\pi: M \rightarrow N$ is a submersion, then for all $p \in N$ the inverse image $\mathcal{F}_{p}=\pi^{-1}(p)$ is a smooth embedded submanifold of $M$, that will be called the fiber at $p$. If $M$ and $N$ are Riemannian manifolds, then a submersion $\pi: M \rightarrow N$ is called a Riemannian submersion if for all $p \in N$ and all $q \in \mathcal{F}_{p}$, the restriction of $\mathrm{d} \pi(q)$ to the orthogonal subspace $T_{q} \mathcal{F}_{p}^{\perp}$ is an isometry onto $T_{p} M$. Given $p \in N$ and $q \in \mathcal{F}_{p}$, a tangent vector $\xi \in T_{q} M$ is said to be vertical if it is tangent to $\mathcal{F}_{p}$, and it is horizontal if it belongs to the orthogonal space $\left(T_{q} \mathcal{F}_{p}\right)^{\perp}$. Let $\mathcal{D}=(T \mathcal{F})^{\perp} \subset T M$ denote the smooth rank $k$ distribution on $M$ consisting of horizontal vectors. The orthogonal distribution $\mathcal{D}^{\perp}$ is clearly integrable, the fibers of the submersion being its maximal integral leaves. Given $\xi \in T M$, its horizontal and vertical components are denoted respectively by $\xi^{h}$ and $\xi^{v}$. The second fundamental form of the fibers is a symmetric tensor $\mathcal{S}^{\mathcal{F}}: \mathcal{D}^{\perp} \times \mathcal{D}^{\perp} \rightarrow \mathcal{D}$, defined by

$$
\mathcal{S}^{\mathcal{F}}(v, w)=\left(\nabla_{v}^{M} W\right)^{h},
$$


where $W$ is a vertical extension of $w$ and $\nabla^{M}$ is the Levi-Civita connection of $M$. In this chapter only we will denote the gradient of a function $u$ by $\operatorname{grad} u$ instead $\nabla u$ to not confuse with the Levi-Civita connection.

For any given vector field $X \in \mathfrak{X}(N)$, there exists a unique horizontal $\widetilde{X} \in \mathfrak{X}(M)$ which is $\pi$-related to $X$, this is, for any $p \in N$ and $q \in \mathcal{F}_{p}$, then $\mathrm{d} \pi_{q}\left(\widetilde{X}_{q}\right)=X_{p}$, called horizontal lifting of $X$. A horizontal vector field $\widetilde{X} \in \mathfrak{X}(M)$ is called basic if it is $\pi$-related to some vector field $X \in \mathfrak{X}(N)$.

If $\widetilde{X}$ and $\widetilde{Y}$ are basic vector fields, then these observations follows easily.

(a) $g^{M}(\tilde{X}, \tilde{Y})=g^{N}(X, Y) \circ \pi$.

(b) $[\widetilde{X}, \widetilde{Y}]^{h}$ is basic and it is $\pi$-related to $[X, Y]$.

(c) $\left(\nabla \widetilde{X} \widetilde{Y}^{M}\right.$ is basic and it is $\pi$-related to $\nabla_{X}^{N} Y$, where $\nabla^{N}$ is the Levi-Civita connection of $g^{N}$.

Let us now consider the geometry of the fibers. First, we observe that the fibers are totally geodesic submanifolds of $M$ exactly when $\mathcal{S}^{\mathcal{F}}=0$. The mean curvature vector of the fiber is the horizontal vector field $H^{3}$ defined by

$$
H(q)=-\sum_{i=1}^{k} \mathcal{S}^{\mathcal{F}}(q)\left(e_{i}, e_{i}\right)=-\sum_{i=1}^{k}\left(\nabla_{e_{i}}^{M} e_{i}\right)^{h},
$$

where $\left(e_{i}\right)_{i=1}^{k}$ is a local orthonormal frame for the fiber through $q$. Observe that $H$ is not basic in general. For instance, when $n=1$, i.e., when the fibers are hypersurfaces of $M$, then $H$ is basic if and only if all the fibers have constant mean curvature. The fibers are minimal submanifolds of $M$ when $H \equiv 0$.

Besides the natural operations of lifting a vector or vector fields in $N$ to horizontal vectors and basic vector fields one has that functions on $N$ can be lifted to functions on $M$ that are constant along the fibers. Such operations preserves the regularity of the lifted objects. One can also (locally) lift curves in the base $\gamma:[a, b] \rightarrow N$ to horizontal curves $\widetilde{\gamma}:[a, c) \rightarrow M$ with the same regularity as $\gamma$ with arbitrary initial condition on the fiber $\mathcal{F}_{\gamma(a)}$. We will need formulas relating the derivatives of $\pi$-related objects in $M$ and $N$. Let us start with divergence of vector fields.

Lemma 4.3. Let $\widetilde{X} \in \mathfrak{X}(M)$ be a basic vector field, $\pi$-related to $X \in \mathfrak{X}(N)$. The following relation holds between the divergence of $\widetilde{X}$ and $X$ at $p \in N$ and $q \in \mathcal{F}_{p}$.

$$
\begin{aligned}
\operatorname{div}^{M}(\widetilde{X})_{q} & =\operatorname{div}^{N}(X)_{p}+g^{M}\left(\widetilde{X}_{q}, H_{q}\right) \\
& =\operatorname{div}^{N}(X)_{p}+g^{N}\left(\mathrm{~d} \pi_{q}\left(\widetilde{X}_{q}\right), \mathrm{d} \pi_{q}\left(H_{q}\right)\right) .
\end{aligned}
$$

In particular, if the fibers are minimal, then $\operatorname{div}^{M}(\widetilde{X})=\operatorname{div}^{N}(X)$.

Formula (59) is obtained by a direct computation of the left-hand side, using a local orthonormal frame $e_{1}, \ldots, e_{k}, e_{k+1}, \ldots, e_{k+n}$ of $T M$, where $e_{1}, \ldots, e_{k}$ are basic fields. The equality follows using equalities (a) and (c) in Subsection 4.1, and formula (58) for the mean curvature.

Given a smooth function $f: N \rightarrow \mathbb{R}$, denote by $\tilde{f}=f \circ \pi: M \rightarrow \mathbb{R}$ its lifting to $M$. It is easy to see that the gradient $\operatorname{grad}^{M} \tilde{f}$ of $\tilde{f}$ is the horizontal lifting of the gradient $\operatorname{grad}^{N} f$. If we denote with a tilde $\widetilde{X}$ the horizontal lifting of a vector field $X \in \mathfrak{X}(N)$, then the previous statement can be written as

$$
\operatorname{grad}^{M} \tilde{f}=\widetilde{\operatorname{grad}^{N}} f \text {. }
$$

\footnotetext{
${ }^{3}$ Sometimes the mean curvature vector is defined as $H(q)=\sum_{i=1}^{k} \mathcal{S}^{\mathcal{F}}(q)\left(e_{i}, e_{i}\right)$
} 
Now, given a function $u: M \rightarrow \mathbb{R}$, one can define a function $u_{\mathrm{av}}: N \rightarrow \mathbb{R}$ by averaging $u$ on each fiber

$$
u_{\mathrm{av}}(p)=\frac{1}{\operatorname{vol} \mathcal{F}_{p}} \cdot \int_{\mathcal{F}_{p}} u \mathrm{~d} \mathcal{F}_{p},
$$

where $\mathrm{d} \mathcal{F}_{p}$ is the volume element of the fiber $\mathcal{F}_{p}$ relative to the induced metric. We are assuming that this integral is finite. As to the gradient of the averaged function $u_{\text {av }}$, we have the following lemma.

Lemma 4.4. Let $p \in N$ and $v \in T_{p} N$ and denote by $V$ the smooth normal vector field along $\mathcal{F}_{p}$ defined by the property $\mathrm{d} \pi_{q}\left(V_{q}\right)=v$ for all $q \in \mathcal{F}_{p}$. Then, for any smooth function $u: M \rightarrow \mathbb{R}$

$$
g^{N}\left(\operatorname{grad}^{N} u_{\mathrm{av}}(p), v\right)=\int_{\mathcal{F}_{p}}\left[g^{M}\left(\operatorname{grad}^{M} u, V\right)+u \cdot g^{M}(H, V)\right] \mathrm{d} \mathcal{F}_{q} .
$$

A standard calculation as in the first variation formula for the volume functional of the fibers. Notice that when $u \equiv 1$, then $u_{\mathrm{av}} \equiv 1$ and (61) reproduces the first variation formula for the volume.

Observe that, in (61), the gradient $\operatorname{grad}^{M} f$ need not be basic or even horizontal ${ }^{4}$. An averaging procedure is available also to produce vector fields $X_{\mathrm{av}}$ on the base out of vector fields $X$ defined in the total space. If $X \in \mathfrak{X}(M)$, let $X_{\text {av }} \in \mathfrak{X}(N)$ be defined by

$$
\left(X_{\text {av }}\right)_{p}=\int_{\mathcal{F}_{p}} \mathrm{~d} \pi_{q}\left(X_{q}\right) \mathrm{d} \mathcal{F}_{p}(q) .
$$

Observe that the integrand above is a function on $\mathcal{F}_{p}$ taking values in the fixed vector space $T_{p} N$. If $X \in \mathfrak{X}(M)$ is a basic vector field, $\pi$-related to the vector field $X_{*} \in \mathfrak{X}(N)$, then $\left(X_{\mathrm{av}}\right)_{p}=\operatorname{vol}\left(\mathcal{F}_{p}\right) \cdot\left(X_{*}\right)_{p}$, where vol denotes the volume. Using the notion of averaged field, equality (61) can be rewritten as

$$
\operatorname{grad}^{N}\left(f_{\text {av }}\right)=\left(\operatorname{grad}^{M} f+f \cdot H\right) \text { av } .
$$

Remark 4.5. From the above formula it follows easily that the averaged mean curvature vector field $H_{\mathrm{av}}$ vanishes at the point $p \in N$ if and only if $p$ is a critical point of the function $z \mapsto \operatorname{vol}\left(\mathcal{F}_{z}\right)$ in $N$. This happens, in particular, when the leaf $\mathcal{F}_{p}$ is minimal. When all the fibers are minimal, or more generally when the averaged mean curvature vector field $H_{\mathrm{av}}$ vanishes identically, then the volume of the fibers is constant.

Corollary 4.6. Let $\pi: M \rightarrow N$ be a Riemannian submersion with compact minimal fibers $\mathcal{F}$. Let $h \in L^{2}(N)$. If $f \in C_{0}^{\infty}(M)$ such that $f_{\mathrm{av}}=0$ for all $q \in N$, then

$$
\int_{M} \widetilde{h} \Delta^{M} f d M=0
$$

Suppose first that $h$ is smooth. By the Divergence Theorem, Fubini's Theorem for Riemannian submersions and 61 we have

$$
\begin{aligned}
\int_{M} \widetilde{h} \Delta^{M} f d M & =-\int_{M} g^{M}\left(\operatorname{grad}^{M} \widetilde{h}, \operatorname{grad}^{M} f\right) d M \\
& =-\int_{N} \int_{\mathcal{F}_{q}} g^{M}\left(\operatorname{grad}^{M} \widetilde{h}, \operatorname{grad}^{M} f\right) d \mathcal{F}_{q} d N \\
& =-\int_{N} g^{N}\left(\operatorname{grad}^{N} h, \operatorname{grad}^{N} f_{\text {av }}\right) d N \\
& =0 .
\end{aligned}
$$

${ }^{4}$ In fact, a gradient is basic if and only if it is horizontal. 
If $h \in L^{2}(N)$ there exists a sequence of smooth functions $h_{k} \in C^{\infty}(N)$ converging to $h$ with respect to the $L^{2}$-norm. On the other hand

$$
\begin{aligned}
\left|\int_{M} \widetilde{h} \Delta^{M} f d M\right| & =\left|\int_{M}\left(\widetilde{h}_{k}-\widetilde{h}\right) \Delta^{M} f d M\right| \\
& \leq \int_{M}\left|\widetilde{h}_{k}-\widetilde{h}\right|\left|\Delta^{M} f\right| d M \\
& \leq\left(\int_{M}\left|\widetilde{h}_{k}-\widetilde{h}\right|^{2} d M\right)^{1 / 2} \cdot\left(\int_{M}\left|\Delta^{M} f\right|^{2} d M\right)^{1 / 2} \\
& =\left\|\Delta^{M} f\right\|_{L^{2}(M)} \cdot\left(\int_{N} \int_{\mathcal{F}_{q}}\left|h_{k}-h\right|^{2} d \mathcal{F}_{q} d M\right)^{1 / 2} \\
& =\operatorname{vol}\left(\mathcal{F}_{q}\right)^{1 / 2} \cdot\left\|\Delta^{M} f\right\|_{L^{2}(M)} \cdot\left\|h_{k}-h\right\|_{L^{2}(N)} .
\end{aligned}
$$

Since $h_{k} \rightarrow h$ in $L^{2}(N)$ then 62 holds. Observe that we used that the volume of the minimal fibers is constant, see Remark 4.5.

Let $\pi: M \rightarrow N$ be a Riemannian submersion. The Laplace-Beltrami operator $\Delta^{N}$ in $N$ of a smooth function $f: N \rightarrow \mathbb{R}$ and the Laplace-Beltrami operator $\Delta^{M}$ in $M$ of its extension $\tilde{f}=f \circ \pi$ are related by the following formula.

Lemma 4.7. Let $f: N \rightarrow \mathbb{R}$ be a smooth function and set $\tilde{f}=f \circ \pi$. Then, for all $p \in N$ and all $q \in \mathcal{F}_{p}$ :

$$
\begin{aligned}
\left(\Delta^{M} \tilde{f}\right)_{q} & =\left(\Delta^{N} f\right)_{p}+g^{M}\left(\left(\operatorname{grad}^{M} \tilde{f}\right)_{q}, H_{q}\right) \\
& =\left(\Delta^{N} f\right)_{p}+g^{N}\left(\left(\operatorname{grad}^{N} f\right)_{p}, \mathrm{~d} \pi_{q}\left(H_{q}\right)\right) .
\end{aligned}
$$

The proof follows easily from (59) applied to the vector fields $X=\operatorname{grad}^{M} \tilde{f}$ and to $X_{*}=$ $\operatorname{grad}^{N} f$, using (60).

4.2. Fundamental tone of a Riemannian submersion. Let $M$ and $N$ be connected Riemannian manifolds and $\pi: M \rightarrow N$ be a Riemannian submersion. Denote by $\Delta^{M}$ and $\Delta^{N}$ the Laplacian operator on functions of $\left(M, g^{M}\right)$ and of $\left(N, g^{N}\right)$ respectively. We want to compare the fundamental tones of open subsets $\Omega \subset N$ with the fundamental tones of its lifting $\widetilde{\Omega}=\pi^{-1}(\Omega)$.

Lemma 4.8. Assume that the fibers of $\pi: M \rightarrow N$ are compact. Let $\Omega$ be an open subset of $N$, and denote by $\widetilde{\Omega}$ the open subset of $M$ given by the inverse image $\pi^{-1}(\Omega)$. Then

$$
\left[\inf _{p \in \Omega} \operatorname{vol}\left(\mathcal{F}_{p}\right)\right] \cdot \lambda^{*}(\widetilde{\Omega}) \leq\left[\sup _{p \in \Omega} \operatorname{vol}\left(\mathcal{F}_{p}\right)\right] \cdot \lambda^{*}(\Omega) .
$$

In particular, if the fibers are minimal, then

$$
\lambda^{*}(\widetilde{\Omega}) \leq \lambda^{*}(\Omega) .
$$

Moreover, if $\inf _{p \in \Omega} \operatorname{vol}\left(\mathcal{F}_{p}\right)>0$ and $\sup _{p \in \Omega} \operatorname{vol}\left(\mathcal{F}_{p}\right)<\infty$ then

$$
\inf _{p \in \Omega} \operatorname{vol}\left(\mathcal{F}_{p}\right) \cdot \inf \sigma_{\text {ess }}(M) \leq \sup _{p \in \Omega} \operatorname{vol}\left(\mathcal{F}_{p}\right) \cdot \inf \sigma_{\text {ess }}(N) .
$$

Proof. Let $\varepsilon>0$ and choose $f_{\varepsilon} \in C_{0}^{\infty}(\Omega)$ such that

$$
\int_{\Omega}\left|\operatorname{grad}^{N} f_{\varepsilon}\right|^{2}<\left(\lambda^{*}(\Omega)+\varepsilon\right) \int_{\Omega} f_{\varepsilon}^{2} .
$$


Consider the function $\tilde{f}_{\varepsilon}=f_{\varepsilon} \circ \pi$. By the assumption that the fibers of $\pi$ are compact, $\tilde{f}_{\varepsilon}$ has compact support in $M$. Using Fubini's Theorem for submersions we have

$$
\int_{\widetilde{\Omega}}\left|\tilde{f}_{\varepsilon}\right|^{2} \mathrm{~d} M=\int_{\Omega}\left(\int_{\mathcal{F}_{p}}\left|\tilde{f}_{\varepsilon}\right|^{2} \mathrm{~d} \mathcal{F}_{p}\right) \mathrm{d} N=\int_{\Omega} \operatorname{vol}\left(\mathcal{F}_{p}\right) \cdot\left|f_{\varepsilon}\right|^{2} \mathrm{~d} N .
$$

Thus

$$
\int_{\widetilde{\Omega}}\left|\tilde{f}_{\varepsilon}\right|^{2} \mathrm{~d} M \geq \inf _{p \in \Omega} \operatorname{vol}\left(\mathcal{F}_{p}\right) \cdot \int_{\Omega}\left|f_{\varepsilon}\right|^{2} \mathrm{~d} N .
$$

Similarly, using (60), we have

$$
\begin{aligned}
\int_{\widetilde{\Omega}}\left|\operatorname{grad}^{M} \tilde{f}_{\varepsilon}\right|^{2} & =\int_{\widetilde{\Omega}}\left|\widetilde{\operatorname{grad}^{N}} f_{\varepsilon}\right|^{2} \\
& =\int_{\Omega}\left(\int_{\mathcal{F}_{p}}\left|\widetilde{\operatorname{grad}^{N}} f_{\varepsilon}\right|^{2} \mathrm{~d} \mathcal{F}_{p}\right) \mathrm{d} N \\
& =\int_{\Omega} \operatorname{vol}\left(\mathcal{F}_{p}\right) \cdot\left|\operatorname{grad}^{N} f_{\varepsilon}\right|^{2},
\end{aligned}
$$

thus

$$
\int_{\widetilde{\Omega}}\left|\operatorname{grad}^{M} \tilde{f}_{\varepsilon}\right|^{2} \leq \sup _{p \in \Omega} \operatorname{vol}\left(\mathcal{F}_{p}\right) \cdot \int_{\Omega}\left|\operatorname{grad}^{N} f_{\varepsilon}\right|^{2} .
$$

Using (67), (69) and (71), we then obtain

$$
\begin{aligned}
\inf _{p \in \Omega} \operatorname{vol}\left(\mathcal{F}_{p}\right) \cdot \lambda^{*}(\widetilde{\Omega}) & \leq \inf _{p \in \Omega} \operatorname{vol}\left(\mathcal{F}_{p}\right) \cdot \frac{\int_{\widetilde{\Omega}}\left|\operatorname{grad}^{M} \tilde{f}_{\varepsilon}\right|^{2}}{\int_{\widetilde{\Omega}}\left|\tilde{f}_{\varepsilon}\right|^{2}} \\
& \leq \sup _{p \in \Omega} \operatorname{vol}\left(\mathcal{F}_{p}\right) \cdot \frac{\int_{\Omega}\left|\operatorname{grad}^{N} f_{\varepsilon}\right|^{2}}{\int_{\Omega}\left|f_{\varepsilon}\right|^{2}} \\
& <\sup _{p \in \Omega} \operatorname{vol}\left(\mathcal{F}_{p}\right) \cdot\left[\lambda^{*}(\Omega)+\varepsilon\right] .
\end{aligned}
$$

This proves (64). If all the fibers are minimal (or more generally if the averaged mean curvature vector field $H_{\text {av }}$ vanishes identically on $N$, see Remark 4.5), then the volume of the fibers is constant, and inequality (65) follows from (64). To prove the inequality (66) we pick a compact subset $K \subset M$ and set $K_{0}=\pi(K)$ and let $\widetilde{K}=\pi^{-1}\left(K_{0}\right)$. The set $\widetilde{K}$ is compact by the assumption that the fibers of $\pi$ are compact. Let $\Omega=N \backslash K_{0}$ and $\widetilde{\Omega}=\pi^{-1}(\Omega)=M \backslash \widetilde{K}$. Clearly, $\widetilde{\Omega} \subset M \backslash K$ and thus $\lambda^{*}(\widetilde{\Omega}) \geq \lambda^{*}(M \backslash K)$. Hence, using (64) we get

$$
\lambda^{*}(M \backslash K) \leq \lambda^{*}(\widetilde{\Omega}) \leq \frac{\sup _{p \in \Omega} \operatorname{vol}\left(\mathcal{F}_{p}\right)}{\inf _{p \in \Omega} \operatorname{vol}\left(\mathcal{F}_{p}\right)} \lambda^{*}(\Omega) \leq \frac{\sup _{p \in \Omega} \operatorname{vol}\left(\mathcal{F}_{p}\right)}{\inf _{p \in \Omega} \operatorname{vol}\left(\mathcal{F}_{p}\right)} \inf \sigma_{\text {ess }}(N) .
$$

Taking the supremum over all compact subset $K \subset M$ in the left-hand side, we obtain the desired inequality.

Now we will consider the case that the fibers of the submersion $\pi: M \rightarrow N$ are compact and minimal.

Lemma 4.9. Let $\pi: M \rightarrow N$ be a Riemannian submersion with compact and minimal fibers $\mathcal{F}$. Then for every open subset $\Omega \subset N$, denoting by $\widetilde{\Omega}$ the inverse image $\pi^{-1}(\Omega)$, one has that

$$
\lambda^{*}(\widetilde{\Omega})=\lambda^{*}(\Omega) .
$$


Proof. In view of $(65)$, it suffices to show the inequality $\lambda^{*}(\widetilde{\Omega}) \geq \lambda^{*}(\Omega)$. To this aim, we will use the estimate in (25). We observe initially that it suffices to prove the inequality when $\Omega$ is bounded. Namely, the general case follows from $\lambda^{*}(\Omega)=\lim _{n \rightarrow \infty} \lambda^{*}\left(\Omega_{n}\right)$, by considering an exhaustion of $\Omega$ by a sequence of bounded open subsets $\Omega_{n}$. Note that $\Omega$ is bounded if and only if $\widetilde{\Omega}$ is bounded, by the compactness of the fibers. Let $f$ be the first eigenfunction of the problem $\Delta^{N} u+\lambda u=0$ in $\Omega$ with Dirichlet boundary conditions, that can be assumed to be positive in $\Omega$.

Set $X=-\operatorname{grad}^{N}(\log f)$, so that $\operatorname{div}^{N}(X)-|X|^{2}=\lambda_{1}(\Omega)$ is constant in $\Omega$. If $\widetilde{X}$ is the horizontal lifting of $X$, then clearly $\left|\widetilde{X}_{q}\right|=\left|X_{\pi(q)}\right|$ for all $q \in \widetilde{\Omega}$. Moreover, by Lemma 4.3, since $H=0, \operatorname{div}^{M}(\tilde{X})_{q}=\operatorname{div}^{N}(X)_{\pi(q)}$. Using (25), we then obtain:

$$
\lambda^{*}(\widetilde{\Omega}) \geq \inf _{\widetilde{\Omega}}\left[\operatorname{div}^{M}(\widetilde{X})-|\widetilde{X}|^{2}\right]=\inf _{\Omega}\left[\operatorname{div}^{N}(X)-|X|^{2}\right]=\lambda^{*}(\Omega)
$$

This proves Lemma (4.9).

Corollary 4.10. Under the assumptions of Lemma 4.9 we have that $\sigma_{\mathrm{ess}}(M)=\emptyset$ if and only if $\sigma_{\mathrm{ess}}(N)=\emptyset$. In particular, if $M$ is a finite covering of $N$, then $\sigma_{\mathrm{ess}}(M) \neq \emptyset$ if and only if $\sigma_{\mathrm{ess}}(N) \neq \emptyset$.

4.3. Proof of Theorem 4.1. The item ii. of Theorem 4.1 follows from Lemma 4.9. For if we take a sequence of compact sets $K_{1} \subset K_{2} \subset \cdots$ with $N=\cup_{i=1}^{\infty} K_{i}$. Likewise we have $M=\cup_{i=1}^{\infty} \widetilde{K}_{i}$, where $\widetilde{K}_{i}=\pi^{-1}\left(K_{i}\right)$. By the proof of (3.1) we have that $\inf \sigma_{\text {ess }}(N)=$ $\lim _{i \rightarrow \infty} \lambda^{*}\left(N \backslash K_{i}\right)$ and $\inf \sigma_{\text {ess }}(M)=\lim _{i \rightarrow \infty} \lambda^{*}\left(M \backslash \widetilde{K}_{i}\right)$. However, $\lambda^{*}\left(N \backslash K_{i}\right)=\lambda^{*}(M \backslash$ $\left.\widetilde{K}_{i}\right)$, by Lemma (4.9). Before we prove item i. we need the following lemma.

Lemma 4.11. Let $\pi: M \rightarrow N$ be a Riemannian submersion with compact minimal fibers $\mathcal{F}$. If $f \in L^{2}(N)$ and $\Delta^{N} f \in L^{2}(N)$ then $\widetilde{f} \in L^{2}(M)$ and $\Delta^{M} \widetilde{f}=\widetilde{\Delta^{N} f} \in L^{2}(M)$. In other words, if $f \in \operatorname{Dom}\left(\Delta^{N}\right)$ then $\widetilde{f} \in \operatorname{Dom}\left(\Delta^{M}\right)$.

Proof. Let $\tilde{f}=f \circ \pi$ be the lifting of $f$. By Fubini's Theorem we have

$$
\int_{M} \widetilde{f}^{2} d M=\int_{N}\left(\int_{\mathcal{F}_{p}} f^{2} d \mathcal{F}_{p}\right) d N=\operatorname{vol}\left(\mathcal{F}_{p}\right) \int_{N} f^{2} d N<\infty
$$

This shows that $\tilde{f} \in L^{2}(M)$. To show that $\Delta^{M} \tilde{f} \in L^{2}(M)$ we have to show that $\Delta^{M} \tilde{f}=$ $\widetilde{\Delta^{N} f}$. Every $\varphi \in C_{0}^{\infty}(M)$ can be decomposed as $\varphi=\varphi_{1}+\varphi_{2}$ where $\varphi_{1}$ is constant along the fibers $\mathcal{F}$ and $\left(\varphi_{2}\right)_{\text {av }}=0$, see [28]. Moreover, $\varphi_{1}$ and $\varphi_{2}$ have compact support. Observe that we can define $\psi: N \rightarrow \mathbb{R}$ by $\psi(\pi(p))=\varphi_{1}(p)$ so that $\varphi_{1}=\widetilde{\psi}$. By Lemma 4.7 we have that $\Delta^{M} \varphi_{1}(p)=\Delta^{N} \psi(\pi(p))$ for every $p \in M$. By Corollary $4.6 \int_{M} \widetilde{f} \Delta^{M} \varphi_{2} d M=0$, 
therefore

$$
\begin{aligned}
& \int_{M} \tilde{f} \Delta^{M} \varphi d M=\int_{M} \tilde{f} \Delta^{M} \varphi_{1} d M \\
& =\int_{N}\left(\int_{\mathcal{F}_{p}} f \Delta^{M} \varphi_{1} d \mathcal{F}_{p}\right) d N \\
& =\int_{N}\left(f \Delta^{N} \psi \int_{\mathcal{F}_{p}} d \mathcal{F}_{p}\right) d N \\
& =\operatorname{vol}\left(\mathcal{F}_{p}\right) \int_{N} f \Delta^{N} \psi d N \\
& =\operatorname{vol}\left(\mathcal{F}_{p}\right) \int_{N} \psi \Delta^{N} f d N \\
& =\int_{N}\left(\int_{\mathcal{F}_{p}} \psi \Delta^{N} f d \mathcal{F}_{p}\right) d N \\
& =\int_{M} \widehat{\psi \Delta^{N}} f d M \\
& =\int_{M} \varphi_{1} \widetilde{\Delta^{N} f} d M \\
& =\int_{M} \varphi \widetilde{\Delta^{N} f} d M
\end{aligned}
$$

To show that $\sigma_{p}(N) \subset \sigma_{p}(M)$ we take $\lambda \in \sigma_{p}(N)$ and $f \in L^{2}(N)$ with $-\Delta^{N} f=\lambda f$ in distributional sense. This implies that $\widetilde{-\Delta^{N} f}=\lambda \tilde{f}$. By Lemma 4.11, $-\Delta^{M} \tilde{f}=\lambda \tilde{f}$ showing that $\lambda \in \sigma_{p}(M)$. To show that $\sigma_{\text {ess }}(N) \subset \sigma_{\text {ess }}(M)$ we take $\mu \in \sigma_{\text {ess }}(N)$. Then, there exists an orthonormal sequence of functions $f_{k} \in \operatorname{Dom}\left(\Delta^{N}\right)$ such that $\|-\Delta^{N} f_{k}-$ $\mu f_{k} \|_{L^{2}(N)} \rightarrow 0$ as $k \rightarrow \infty$. By Lemma 4.11, we have that $\widetilde{f_{k}} \in \operatorname{Dom}\left(\Delta^{M}\right)$. Now

$$
\begin{aligned}
\left\|-\Delta^{M} \tilde{f}_{k}-\mu \tilde{f}_{k}\right\|_{L^{2}(M)}^{2} & =\int_{M}\left|-\Delta^{M} \tilde{f}_{k}-\mu \tilde{f}_{k}\right|^{2} d M \\
& =\int_{N} \int_{\mathcal{F}_{q}}\left|-\Delta^{N} f_{k}-\mu f_{k}\right|^{2} d \mathcal{F}_{q} d N \\
& =\operatorname{vol}\left(\mathcal{F}_{q}\right) \int_{N}\left|-\Delta^{N} f_{k}-\mu f_{k}\right|^{2} d N \\
& =\operatorname{vol}\left(\mathcal{F}_{q}\right)\left\|-\Delta^{N} f_{k}-\mu f_{k}\right\|_{L^{2}(N)}^{2} \rightarrow 0 .
\end{aligned}
$$

This shows that $\mu \in \sigma_{\mathrm{ess}}(M)$, the proof of Theorem 4.1 is concluded.

Corollary 4.12. Let $G$ be a Lie group endowed with a bi-invariant metric. Then, $\sigma_{\mathrm{ess}}(G)$ is empty if and only if for some (hence for any) compact subgroup $K \subset G$, the Riemannian homogeneous space $G / K$ has empty essential spectrum.

Apply Theorem 4.1 to the Riemannian submersion $G \mapsto G / K$, which has minimal and compact fibers.

Other interesting examples of applications of Theorem 4.1 arise from non compact Lie groups. Consider the $2 \times 2$ special linear group $\mathrm{SL}(2, \mathbb{R})$. There exists a 2-parameter family of left-invariant Riemannian metrics $g_{\kappa, \tau}$, with $\kappa<0$ and $\tau \neq 0$, for which $\left(\mathrm{SL}(2, \mathbb{R}), g_{\kappa, \tau}\right) \rightarrow \mathbb{H}_{\kappa}^{2}$ is a Riemannian submersion with geodesic fibers diffeomorphic to the circle $\mathbb{S}^{1}$. An explicit description of these metrics can be found, for instance, in [134]. Endowed with these metrics, $\operatorname{SL}(2, \mathbb{R})$ is one of the eight homogeneous Riemannian 3 -geometries, as classified in [126], and its isometry group has dimension 4. 
Proposition 4.13. For all $\kappa<0$ and $\tau \neq 0$,

$$
\sigma\left(\mathrm{SL}(2, \mathbb{R}), g_{\kappa, \tau}\right)=\sigma_{\mathrm{ess}}\left(\mathrm{SL}(2, \mathbb{R}), g_{\kappa, \tau}\right)=\left[-\frac{\kappa}{4},+\infty\right) .
$$

It is known that the spectrum $\sigma\left(\mathbb{H}_{\kappa}\right)=\sigma_{\text {ess }}\left(\mathbb{H}_{\kappa}\right)=\left[-\frac{\kappa}{4},+\infty\right)$, see [52]. By Lemma 4.9

$$
\lambda^{*}\left(\mathrm{SL}(2, \mathbb{R}), g_{\kappa, \tau}\right)=\lambda^{*}\left(\mathbb{H}_{\kappa}\right)=-\frac{\kappa}{4},
$$

hence $\sigma\left(\operatorname{SL}(2, \mathbb{R}), g_{\kappa, \tau}\right) \subset\left[-\frac{\kappa}{4},+\infty\right)$. On the other hand, by Theorem 4.1

$$
\left[-\frac{\kappa}{4},+\infty\right)=\sigma_{\mathrm{ess}}\left(\mathbb{H}_{\kappa}\right) \subset \sigma_{\mathrm{ess}}\left(\mathrm{SL}(2, \mathbb{R}), g_{\kappa, \tau}\right) .
$$

This proves the proposition.

\section{Calabi-Yau conjectures and discrete spectrum}

A particularly interesting problem in Riemannian Geometry is the spectrum related part of the Calabi-Yau conjectures on minimal hypersurfaces. The Calabi-Yau conjectures have their origin in two problems proposed by E. Calabi in the 1960's, about the nonexistence of complete minimal hypersurfaces of $\mathbb{R}^{n}$ subjected to certain extrinsic bounds ([30], see also [44, p. 212]).

- Calabi proposed the first conjecture as an exercise. He wrote: "Prove that any complete minimal hypersurface in $\mathbb{R}^{n}$ must be unbounded."

- The second problem, on the other hand, was proposed almost as an unlikely conjecture. He wrote "A more ambitious conjecture is: a complete minimal hypersurface in $\mathbb{R}^{n}$ has an unbounded projection in every $(n-2)$-dimensional flat subspace."

It is known by the work of L. Jorge-F. Xavier [84] and N. Nadirashvili [107] that both conjectures turned out to be false. More precisely, Jorge-Xavier constructed a non-flat, complete minimal surface lying between two parallel planes in $\mathbb{R}^{3}$, showing that the second conjecture was false in general, whereas N. Nadirashvili constructed a bounded, complete minimal immersion of the unit disk $\mathbb{D}$ into $\mathbb{R}^{3}$, contradicting the statement of the first conjecture. In his Millennium Lectures [138], [139], S. T. Yau, revisiting the E. Calabi conjectures on the existence of bounded minimal surfaces, [30], [44], in the light of JorgeXavier and Nadirashvili's counter-examples, [84], [107] proposed a new set of questions about bounded minimal surfaces of $\mathbb{R}^{3}$. Among the new questions one regards the spectrum of bounded minimal surfaces, and among them he asked whether the spectrum of bounded minimal surfaces of $\mathbb{R}^{3}$ was discrete. Yau's questions motivated the construction of a large number of exotic examples of minimal surfaces in $\mathbb{R}^{3}$ that followed from Jorge-Xavier and Nadirashvili's methods, see [2], [3], [4], [63], [92], [93], [99], [100], [101], [102], [132].

In this section we shed light on the essential spectrum of bounded submanifolds, in particular, the spectrum of many examples constructed after the Calabi-Yau conjectures. The new fact we found is that the size of the limit sets of bounded immersions plays an important role in the existence of elements in their essential spectrum. Recall that the limit set of an isometric immersion $\varphi: M \rightarrow \Omega \subset N$ is the set

$$
\lim \varphi:=\left\{y \in \bar{\Omega} ; \exists\left\{x_{n}\right\} \subseteq M \text { divergent in } M \text {, s.t. } \varphi\left(x_{n}\right) \rightarrow y \text { in } N\right\},
$$

and that $\varphi$ is proper in $\Omega$ if and only if $\lim \varphi \subset \partial \Omega$. We briefly recall the main sets of examples that our main result applies.

(i.) For each convex domain $\Omega \subseteq \mathbb{R}^{3}$, not necessarily bounded or smooth, Martin and Morales constructed a complete minimal disk $\varphi: \mathbb{D} \hookrightarrow \Omega$ properly immersed into $\Omega$, see [99]. 
(ii.) M. Tokuomaru [132], constructed a complete minimal annulus $\varphi: A \hookrightarrow \mathbb{R}^{3}$ properly immersed into the unit ball of $B_{\mathbb{R}^{3}}(1) \subset^{3}$.

(iii.) Martin and Morales [100], improved the results of [99], showing that, if $\Omega$ is a bounded, strictly convex domain of $\mathbb{R}^{3}$, with $\partial \Omega$ of class $C^{2, \alpha}$, then there exists a complete, minimal disk properly immersed into $\Omega$ whose limit set is closed to a prescribed Jordan curve ${ }^{5}$ on $\partial \Omega$.

(iv.) A. Alarcon, L. Ferrer and F. Martin [3, Thm B.], extended the results of [99] and [132]. They showed that for any convex domain $\Omega \subset \mathbb{R}^{3}$, not necessarily bounded or smooth, there exists a proper minimal immersion $\varphi: M \rightarrow \Omega$ of a complete non-compact surface $M$ with arbitrary finite topology into $\Omega$.

(v.) Ferrer, Martin and Meeks [63], improved the main results of [100], proving that given a bounded smooth domain $\Omega \subset \mathbb{R}^{3}$ and given any open surface $M$, there exists a complete, proper, minimal immersion $\varphi: M \rightarrow \Omega$ with the property that the limit sets of different ends are disjoint, compact, connected subsets of $\partial \Omega$. It should be remarked that the Ferrer-Martin-Meeks' surfaces [63] immersed into a bounded smooth domain $\Omega$ can have either finite or infinite topology. They can have uncountably many ends and be either orientable or non-orientable. Moreover, the convexity of $\Omega$ is not a necessary hypothesis, although they need its smoothness. In fact, one can not drop the convexity and the smoothness of $\Omega$ altogether, see [98] for a counter-example. They also prove that for every convex open set $\Omega$ and every non-compact, orientable surface $M$, there exists a complete, proper minimal immersion $\varphi: M \rightarrow \Omega$ such that $\lim \varphi \equiv \partial \Omega$, see [63, Prop.1].

(vi.) F. Martin and N. Nadirashvili [102], constructed complete minimal immersions $\varphi: \mathbb{D} \rightarrow \mathbb{R}^{3}$ of the disk $\mathbb{D} \subset \mathbb{C}$, admitting continuous extensions $\bar{\varphi}: \overline{\mathbb{D}} \rightarrow \mathbb{R}^{3}$ such that $\bar{\varphi}_{\mid \partial \mathbb{D}}: \partial \mathbb{D}=\mathbb{S}^{1} \rightarrow \bar{\varphi}\left(\mathbb{S}^{1}\right)$ is an homeomorphism and $\bar{\varphi}\left(\mathbb{S}^{1}\right)$ is a non-rectifiable Jordan curve of Hausdorff dimension $\operatorname{dim}_{\mathcal{H}}\left(\bar{\varphi}\left(\mathbb{S}^{1}\right)\right)=1$. They also showed that the set of Jordan curves $\bar{\varphi}\left(\mathbb{S}^{1}\right)$ constructed via this procedure is dense in the space of Jordan curves of $\mathbb{R}^{3}$ with respect to the Hausdorff metric.

(vii.) Alarcon proved in [2] that for any arbitrary finite topological type there exists a compact Riemann surface $\mathcal{M}$, an open domain $M \subset \mathcal{M}$ with this topological type and a conformal complete minimal immersion $\varphi: M \rightarrow \mathbb{R}^{3}$ that admits an extension to a continuous map $\bar{\varphi}: \bar{M} \rightarrow \mathbb{R}^{3}$ such that $\bar{\varphi}_{\mid \partial M}$ is an embedding and the Hausdorff dimension of $\bar{\varphi}(\partial M)$ is 1 .

We will address S. T. Yau's question whether the spectrum of bounded minimal surfaces of $\mathbb{R}^{3}$ is discrete or not. Bessa-Jorge-Montenegro in [19] gave a first answer to this question, that applies to some of the examples quoted above.

Theorem 5.1 (Bessa-Jorge-Montenegro [19]). Let $\varphi: M \hookrightarrow B_{N}(r)$ be a complete $m$ submanifold properly immersed into a geodesic ball of a Riemannian $n$-manifold $N$, with center at $p$ and radius $r$. Let $b=\sup K_{N}^{\mathrm{rad}}$, where $K_{N}^{\mathrm{rad}}$ are the radial sectional curvatures along the geodesics issuing from $p$. Assume that $r<\min \left\{\operatorname{inj}_{N}(p), \pi / 2 \sqrt{b}\right\}$, where $\pi / 2 \sqrt{b}=$ $+\infty$ if $b \leq 0$. If the norm of the mean curvature vector $H$ satisfies,

$$
\sup _{M}|H|<m \cdot C_{b}(r)
$$

then $M$ has discrete spectrum.

Here

$$
C_{b}(t)= \begin{cases}\sqrt{b} \cot (\sqrt{b} t), & \text { if } \quad b>0 \\ 1 / t, & \text { if } \quad b=0 \\ \sqrt{-b} \operatorname{coth}(\sqrt{-b} t), & \text { if } \quad b<0\end{cases}
$$

\footnotetext{
${ }^{5}$ A continuous embedding $\gamma: \mathbb{S}^{1} \rightarrow \mathbb{R}^{3}$.
} 
Theorem 5.1 is consequence of a simpler general result.

Theorem 5.2 (Bessa-Jorge-Montenegro). If $f: M \rightarrow\left[0, \Lambda^{2}\right)$ is a proper, $C^{2}$-smooth function satisfying

$$
\liminf _{\rho(x) \rightarrow \infty} \Delta f \geq c>0
$$

then $\sigma_{\mathrm{ess}}(M)=\emptyset$. Here $\rho(x)=\operatorname{dist}_{M}(x, o), o \in M$.

Proof. First, recall that $\sigma_{\text {ess }}(M)=\emptyset \Leftrightarrow \lim _{i \rightarrow \infty} \lambda^{*}\left(M \backslash K_{i}\right)=\infty$ for any exhaustion sequence of $M$ by compact sets $K_{1} \subset K_{2} \subset \ldots \stackrel{i \rightarrow \infty}{\subset} K_{i} \subset \ldots$, see Proposition 3.1. Since $f$ is proper the sets $\left\{K_{i}=f^{-1}([0, \sup f-1 / i])\right\}$ form an exhaustion sequence of compact subsets of $M$. We need to estimate $\lambda^{*}\left(M \backslash K_{i}\right)$ from below. Let $g: M \rightarrow[0, \sup f]$ be a smooth function defined by $g(x)=\sup f-f(x) \geq 0$. By the generalized Barta's Theorem 2.23 we have that

$$
\lambda^{*}\left(M \backslash K_{i}\right) \geq \inf _{M \backslash K_{i}}-\frac{\Delta g}{g}=\inf _{M \backslash K_{i}} \frac{\Delta f}{\sup f-f} \geq \frac{c}{1 / i}=i \cdot c
$$

Thus $\lim _{i \rightarrow \infty} \lambda^{*}\left(M \backslash K_{i}\right)=\infty$.

Proof of Theorem 5.1. It suffices to produce a proper, bounded above, non-negative, smooth function $f: M \rightarrow\left[0, \Lambda^{2}\right)$ satisfying $\liminf _{\rho(x) \rightarrow \infty} \Delta f \geq c>0$. Let $\varphi: M \rightarrow B_{N}(r)$ be a proper isometric immersion of a complete Riemannian $m$-manifold into a normal geodesic ball of radius $r<\min \{\operatorname{inj}(p), \pi / 2 \sqrt{b}\}$ of a Riemannian manifold $N$, where $p$ is the center of the $B_{N}(r)$ and $b=\sup _{B_{N}(r)} K^{\mathrm{rad}}$, assuming that $\pi / 2 \sqrt{b}=\infty$ if $b \leq 0$. By the hessian comparison theorem [69] we have that for $\rho_{N}(x)=\operatorname{dist}_{N}(p, x)$

$$
\operatorname{Hess}_{N} \rho_{N}(x)(X, X) \geq C_{b}\left(\rho_{N}(x)\right)\left\{|X|^{2}-\langle X, \nabla \rho\rangle^{2}\right\} .
$$

Define a function $v: B_{N}(r) \rightarrow \mathbb{R}$ by $v(y)=\phi_{b}(\rho(y))$, where $\phi_{b}:[0, r] \rightarrow \mathbb{R}$ given by

$$
\phi_{b}(t)= \begin{cases}{[1-\cos (\sqrt{b} t)] / b,} & \text { if } b>0, \quad t<\pi / 2 \sqrt{b}, \\ t^{2} / 2, & \text { if } b=0 \\ {[\cosh (\sqrt{-b} t)-1] /(-b),} & \text { if } b<0\end{cases}
$$

Observe that $\phi(t)>0$ in $[0, r), \phi_{b}(0)=0, \phi_{b}^{\prime}(t)>0$ and $\phi_{b}^{\prime \prime}(t)-C_{b}(t) \phi_{b}^{\prime}(t)=0$ in $[0, r]$. Let $f: M \rightarrow \mathbb{R}$ defined by $f=v \circ \varphi \in\left[0, \phi_{b}(r)\right]$. The function $f$ is smooth, non-negative, bounded above and proper. Let us compute $\Delta f$. Computing $\Delta f$ using Jorge-Koutrofiotis formula [82] we have

$$
\begin{aligned}
\Delta f(x) & =\sum_{i=1}^{m} \operatorname{Hess}_{N} v(\varphi(x))\left(e_{i}, e_{i}\right)+\left\langle\nabla v, \sum_{i=1}^{m} \alpha\left(e_{i}, e_{i}\right)\right\rangle \\
& =\sum_{i=1}^{m} \operatorname{Hess}_{N} v(\varphi(y))\left(e_{i}, e_{i}\right)+\langle\nabla v, H\rangle .
\end{aligned}
$$

Where $\left\{e_{i}\right\}$ is an orthonormal basis of $T_{x} M$. Computing $\operatorname{Hess}_{N} v(\varphi(x))\left(e_{i}, e_{i}\right)$ we have, setting $t=\rho_{N}(\varphi(x))$,

$$
\begin{aligned}
\operatorname{Hess}_{N} v(\varphi(x))\left(e_{1}, e_{1}\right) & =\phi_{b}^{\prime \prime}(t)\left\langle e_{i}, \nabla \rho_{N}\right\rangle^{2}+\phi_{b}^{\prime}(t) \operatorname{Hess}_{N} \rho_{N}\left(e_{i}, e_{i}\right) \\
& \geq \phi_{b}^{\prime \prime}(t)\left\langle e_{i}, \nabla \rho_{N}\right\rangle^{2}+\phi_{b}^{\prime}(t) C_{b}(t)\left[1-\left\langle e_{i}, \nabla \rho_{N}\right\rangle^{2}\right] \\
& =\phi_{b}^{\prime}(t) C_{b}(t) \\
& = \begin{cases}\cos (\sqrt{b} t), & \text { if } b>0, \\
t, & \text { if } b=0, \\
\cosh (\sqrt{-b} t), & \text { if } b<0 .\end{cases}
\end{aligned}
$$


Therefore,

$$
\begin{aligned}
\Delta f(x) & \geq m \cdot \phi_{b}^{\prime}(t) C_{b}(t)-\phi_{b}^{\prime}(t) \sup |H| \\
& =\phi_{b}^{\prime}(t)\left[m \cdot C_{b}(t)-\sup |H|\right] .
\end{aligned}
$$

Since $f$ is proper we have that $f(x) \rightarrow \phi_{b}(r)$ when $\rho(x)=\operatorname{dist}(o, x) \rightarrow \infty$. Therefore $\Delta f(x) \geq \phi_{b}^{\prime}(t)\left[m \cdot C_{b}(t)-\sup |H|\right] \rightarrow \phi_{b}^{\prime}(r)\left[m \cdot C_{b}(r)-\sup |H|\right]>0$, when $\rho(x) \rightarrow \infty$. Set $c=\phi_{b}^{\prime}(r)\left[m \cdot C_{b}(r)-\sup |H|\right]$ and Theorem 5.1 is proved.

Despite the generality of this result, the "bounding" convex domains $\Omega \subset \mathbb{R}^{3}$ were restricted to geodesic balls. Moreover, properness was used in a fundamental way such that it cannot be modified to deal with non-proper immersions. Here we will provide a sharp, general criterion that applies to each of the examples in (i.), ..., (vii.) and answers Yau's question. In Theorem 5.6, we show that the spectrum of a bounded minimal surface is discrete provided its limit set has zero Hausdorff measure of order $\Psi(t)=t^{2}|\log t|$. Moreover, we consider bounded immersions where the "bounding" set satisfies a weaker convexity notion. On the other hand, the universal covering of Tokuomaru bounded properly immersed minimal annulus $\varphi: A \rightarrow \mathbb{R}^{3}$, item (ii) [132], has limit set with Hausdorff dimension 2 and by Theorem 6.13 it has essential spectrum not empty.

Definition 5.3. An open subset $\Omega \subset N^{n}$ with smooth $C^{2}$-boundary is strictly $j$-convex, $j \in\{1, \ldots, n-1\}$, if for every $q \in \partial \Omega$, the ordered eigenvalues $\xi_{1}(q) \leq \cdots \leq \xi_{n-1}(q)$ of the second fundamental form $\alpha$ of the boundary $\partial \Omega$ at $q$ with respect to the unit normal vector field $\nu$ pointing towards $\Omega$ satisfies $\xi_{1}(q)+\ldots+\xi_{j}(q)>0$. If for all $q \in \partial \Omega$ and some constant $c>0$, the eigenvalues satisfy $\xi_{1}(q)+\ldots+\xi_{j}(q) \geq c$, then we say that $\Omega$ is strictly $j$-convex with constant $c$.

A result of J. Hadamard [76], states that if a compact immersed hypersurface $M \subset \mathbb{R}^{n}$ has positive definite second fundamental form at all $p \in M$, then $M$ is embedded as the boundary $M=\partial \Omega$ of a strictly convex body $\Omega$. In other words, a compact 1-convex subset $\Omega \subset \mathbb{R}^{n}$ is a convex body in the sense that any two points in $\Omega$ can be joined by a segment contained in $\Omega$. The classical notions of convexity and mean convexity are respectively 1-convexity and $(n-1)$-convexity. The following example due to Jorge-Tomi [83] shows that a set can be 2-convex without being 1-convex. Let

$$
\mathbb{T}^{n}\left(r_{1}, r_{2}\right)=\left\{(z, w) \in \mathbb{R}^{2} \times \mathbb{R}^{n-2}:\left(|z|-r_{2}\right)^{2}+|w|^{2} \leq r_{1}^{2}\right\}, \quad 0<r_{1}<r_{2}
$$

be the solid torus homeomorphic to $\mathbb{S}^{1} \times \mathbb{B}^{n-1}$, where $\mathbb{B}^{n-1}$ is the unit ball of $\mathbb{R}^{n-1}$. It was shown in [83] that $\mathbb{T}^{n}$ is 2-convex whenever this relation $r_{1} \leq r_{2} / 2$ is satisfied. Regarding these notions of $j$-convexity, we shall show that strictly $j$-convexity of an open set $\Omega$ with constant $c>0$ and $C^{3}$-smooth boundary $\partial \Omega$ is equivalent to the existence of suitable $j$-subharmonic $C^{2}$-function $f: \Omega \rightarrow \mathbb{R}$, see Lemma 5.13 for details.

Definition 5.4. Let $\Omega \subset N$ be a bounded open set in a Riemannian manifold. For a given $r>0$ let $T_{r}(\Omega)=\left\{y \in N: \operatorname{dist}_{N}(y, \Omega) \leq r\right\}$ be the closed tube around $\Omega$ and let

$$
b=\sup \left\{K_{N}(z), z \in T_{\operatorname{diam}(\Omega)}(\Omega)\right\} .
$$

For each $y \in \Omega$ define $r(y)=\min \left\{\operatorname{inj}_{N}(y), \pi / 2 \sqrt{b}\right\}$, where $\pi / 2 \sqrt{b}$ is replaced by $+\infty$ if $b \leq 0$. Set $r_{\Omega}=\inf _{y \in \Omega} r(y)$. A bounded domain $\Omega \subset N$ is said to be totally regular if $\operatorname{diam}_{N}(\Omega)<r_{\Omega}$.

Example 5.5. Any bounded domain $\Omega \subset N$ of a Hadamard manifold is totally regular. On the other hand, $\Omega \subset \mathbb{S}^{n}(1)$ is totally regular if and only if $\operatorname{diam}_{\mathbb{S}^{n}(1)}(\Omega)<\pi / 2$. 
For $b \in \mathbb{R}$, define the function $\mu_{b}:[0, \infty) \rightarrow \mathbb{R}$ by

$$
\mu_{b}(t)=\left\{\begin{array}{ccc}
\frac{1}{\sqrt{b}} \tan (\sqrt{b} t), & \text { if } \quad b>0 \\
t, & \text { if } \quad b=0 \\
\frac{1}{\sqrt{-b}} \tanh (\sqrt{-b} t), & \text { if } \quad b<0
\end{array}\right.
$$

See [105, Chapter 4] for the definition of generalized Hausdorff measures or simply the $\Psi$-Hausdorff measures $\mathcal{H}_{\Psi}$, where $\Psi:[0, \infty) \rightarrow[0, \infty)$ is a continuous function. Our first result in this section is the following theorem that gives sufficient conditions on the size of the limit set of a bounded submanifold for its spectrum to be discrete.

Theorem 5.6 (Bessa-Jorge-Mari-[17]). Let $\varphi: M \rightarrow N$ be an isometric immersion of a Riemannian $m$-manifold $M$ into a Riemannian $n$-manifold $N$ with mean curvature vector $H$. Let us suppose that $\varphi(M) \subset \Omega$, is a bounded, totally regular, open subset of $N$ and let $b$ be as in (78) and $\mu_{b}$ as defined in (79). Assume that

$$
\|H\|_{L^{\infty}(M)}<\frac{m-1}{m \cdot \mu_{b}(\operatorname{diam}(\Omega))} .
$$

Define $\theta=\left[m-1-m \cdot \mu_{b}(\operatorname{diam}(\Omega)) \cdot\|H\|_{L^{\infty}(M)}\right]>0$ and $\Psi \in C^{0}([0, \infty)$ given by

$$
\Psi(t)= \begin{cases}t^{2}, & \text { if } \quad \theta>1 \\ t^{2}|\log t|, & \text { if } \quad \theta=1 \\ t^{\theta+1}, & \text { if } \quad \theta \in(0,1) .\end{cases}
$$

If one of the following conditions holds

(1) $\lim \varphi \cap \partial \Omega=\emptyset$ and $\mathcal{H}_{\Psi}(\lim \varphi)=0$,

(2) $\lim \varphi \cap \partial \Omega \neq \emptyset, \mathcal{H}_{\Psi}(\lim \varphi \cap \Omega)=0, \Omega$ is strictly $m$-convex with constant $c>0$, $\partial \Omega$ is of class $C^{3}$, and the mean curvature vector $H$ satisfies the further restriction

$$
\|H\|_{L^{\infty}(M)}<\frac{c}{m}
$$

then the spectrum of $-\Delta$ is discrete.

We shall make few comments about Theorem 5.6.

- We remark that in item 2 , the Hausdorff measure of $\lim \varphi \cap \partial \Omega$ does not need to be zero. In particular, the examples of Ferrer, Martin, Meeks [63] of complete, proper minimal immersions $\varphi: M \rightarrow \Omega$ such that $\lim \varphi \equiv \partial \Omega \subset \mathbb{R}^{3}$ have discrete spectrum, provided $\Omega$ is strictly 2 -convex. One illustrative example is the 2 -convex solid torus $\mathbb{T}^{2}\left(r_{1}, r_{2}\right), r_{1} \leq r_{2} / 2$ described in [83]. If $M$ is any open surface, then there exists a complete, proper minimal immersion $\varphi: M \rightarrow \mathbb{T}^{2}\left(r_{1}, r_{2}\right)$, [63, Prop. $1]$, such that $\lim \varphi \equiv \partial \mathbb{T}^{2}\left(r_{1}, r_{2}\right)$, hence by Theorem 5.6, item 2, its spectrum is discrete.

- Our definition of $\Omega$ being totally regular implies that $\mu_{b}(\operatorname{diam}(\Omega))>0$ thus $(80)$ is meaningful, where $b=\sup \left\{K_{N}(z), z \in T_{\operatorname{diam}(\Omega)}(\Omega)\right\}$. However, if one knows only an upper bound for the sectional curvatures $b_{0}>b$ instead, then Theorem 5.6 is still valid, provided $\mu_{b_{0}}(\operatorname{diam}(\Omega))>0$.

- The case that $\lim \varphi \cap \Omega=\emptyset$ is equivalent to the properness of $\varphi$ in $\Omega$, therefore the statement of Theorem 5.6 extends, (in many aspects), the main result of [19].

- Theorem 5.6 also applies to non-orientable manifolds $M$. In fact, its proof can be applied to the two-sheeted oriented covering of $M$ yielding the same conclusions.

- The Riemannian manifold $M$ may be geodesically incomplete and the statement regards the spectrum of the Friedrichs extension of $\Delta: C_{c}^{\infty}(M) \rightarrow C_{c}^{\infty}(M)$. 
The minimal surfaces in the examples (i.),(ii.), (iii.) and (iv.) are properly immersed in 1-convex domains $\Omega$ of $\mathbb{R}^{3}$, whereas the minimal surfaces in (v.) are properly immersed in smooth domains $\Omega$. In those examples $\lim \varphi \cap \Omega=\emptyset$ thus $\mathcal{H}_{\Psi}(\lim \varphi \cap \Omega)=0$. The examples in (vi.) and (vii.) are bounded and $\lim \varphi$ is a non-rectifiable Jordan curve of Hausdorff dimension 1. Thus $\mathcal{H}_{\Psi}(\lim \varphi \cap \Omega)=0$ for $\Psi(t)=t^{2}|\log (t)|$. By Theorem 5.6, all of those examples of (i.), (ii.), (iii.), (iv.), (v.), (vi).) and (vii.) have discrete spectrum, provided $\Omega$ is bounded strictly 2 -convex with $C^{3}$-boundary. That can be summarized in the following corollary as follows.

Corollary 5.7. Let $\varphi: M^{m} \rightarrow N^{n}$ be a minimal m-submanifold, possibly incomplete, immersed into a bounded open $m$-convex subset $\Omega$ of a Hadamard manifold with constant $c>0$. Suppose that $\partial \Omega$ is $C^{3}$-smooth and $\Psi(t)=t^{2}$ if $m \geq 3$ and $\Psi(t)=t^{2}|\log (t)|$ if $m=2$. If $\mathcal{H}_{\Psi}(\lim \varphi \cap \Omega)=0$, then the spectrum of $-\Delta$ is discrete. In particular, those minimal surfaces constructed in (i.), (ii.), (iii), (iv.), (v.), (vi.) and (vii.) have discrete spectrum provided $\Omega$ is bounded, strictly 2-convex with $C^{3}$-boundary.

Remark 5.8. The hypothesis concerning the measure of the limit set $\lim \varphi$ in Theorem 5.6 is sharp. Consider a bounded, complete proper minimal annulus $\varphi: M \rightarrow B_{1}^{\mathbb{R}^{3}}(0)$ as in [132] with $\lim \varphi \cap \Omega=\emptyset$, thus with discrete spectrum by Theorem 5.6 or $[19$, Thm.1]. Considering the universal cover $\pi: \widetilde{M} \rightarrow M$ and setting $\phi=\varphi \circ \pi: \widetilde{M} \rightarrow \mathbb{R}^{3}$ one has a bounded, complete minimal surface with non-empty essential spectrum. In fact, if $\pi:\left(\widetilde{M}, \pi^{*} \mathrm{~d} s^{2}\right) \rightarrow\left(M, \mathrm{~d} s^{2}\right)$ is an infinite sheeted covering then the induced metric $\pi^{*} \mathrm{~d} s^{2}$ satisfies the "ball property", see Definition 6.11, therefore the essential spectrum of $\left(\widetilde{M}, \pi^{*} d s^{2}\right)$ is non-empty, regardless the spectrum of $\left(M, d s^{2}\right)$. Observe that the immersed submanifold have the same image $\varphi(M)=\phi(\widetilde{M})$ however, their limit sets are different, $\lim \varphi \neq \lim \phi=\overline{\phi(M)}$ and Theorem 5.6 could not be applied since the Hausdorff dimension $\operatorname{dim}_{\mathcal{H}}\left(\lim \phi \cap B_{1}^{\mathbb{R}^{3}}(0)\right) \geq 2$.

\subsection{Proof of Theorem 5.6.}

5.1.1. Preliminaries. We will denote by $\varphi: M \rightarrow N$ an isometric immersion of a complete Riemannian $m$-manifold $M$ into a Riemannian $n$-manifold $N$. The Levi-Civita connections of $N$ and $M$ are denoted by $\bar{\nabla}$ and $\nabla$ respectively. The second fundamental form $\alpha=$ $\bar{\nabla} \mathrm{d} \varphi^{\perp}$ and mean curvature vector $H=\operatorname{tr} \alpha / m$. The gradient of a function $g: N \rightarrow \mathbb{R}$, is denoted by $\bar{\nabla} g$ whereas $\nabla(g \circ \varphi)=(\bar{\nabla} g)^{\top}$ is the gradient of $g \circ \varphi$, the restriction of $g$ to $M$. The hessian of $g$ is denoted by $\bar{\nabla} \mathrm{d} g$ and the hessian $\nabla \mathrm{d}(g \circ \varphi)$ of $g \circ \varphi$ are related by

$$
\nabla \mathrm{d}(g \circ \varphi)=\bar{\nabla} \mathrm{d} g+\left\langle\bar{\nabla} \mathrm{d} \varphi^{\perp}, \bar{\nabla} g\right\rangle .
$$

The symbol $B_{r}^{N}(x)$ denotes the geodesic ball of $N$ centered at $x \in N$ with radius $r$. However the unit ball $B_{1}^{\mathbb{R}^{2}}(0)$ of $\mathbb{R}^{2}$, will be denoted by $\mathbb{D}$. Similarly, for $X \subset N$ the symbol $T_{r}^{N}(X)$, called the tube of radius $r$ around $X$, denotes the open set of points (in $N$ ) whose distance from $X$ is less than $r$. Finally, denote by $\mathbb{R}^{+}=(0,+\infty)$ and $\mathbb{R}_{0}^{+}=[0,+\infty)$.

5.1.2. Carathéodory's Construction. In this section we shall review the notion of generalized $\Psi$-Hausdorff measures. Here, we do follow the elegant exposition of P. Mattila, in [105, Chap.4].

Definition 5.9 (Carathéodory's Construction). Let $X$ be a metric space, $\mathcal{J}$ a family of subsets of $X$ and $\zeta \geq 0$ a non-negative function on $\mathcal{J}$. Make the following assumptions.

1. For every $\delta>0$ there are $E_{1}, E_{2}, \ldots, \in \mathcal{J}$ such that $X=\bigcup_{i=1}^{\infty} E_{i}$ and $\operatorname{diam}\left(E_{i}\right) \leq$ $\delta$.

2. For all $\delta>0$ there is $E \in \mathcal{J}$ such that $\zeta(E) \leq \delta$ and $\operatorname{diam}(E) \leq \delta$. 
For $0<\delta \leq \infty$ and $A \subset X$ we define

$$
\zeta_{\delta}(A)=\inf \left\{\sum_{i=1}^{\infty} \zeta\left(E_{i}\right): A \subset \bigcup_{i=1}^{\infty} E_{i}, \operatorname{diam}\left(E_{i}\right) \leq \delta, E_{i} \in \mathcal{J}\right\} .
$$

It is easy to see that $\zeta_{\delta}(A) \leq \zeta_{\epsilon}(A)$ whenever $0<\epsilon<\delta \leq \infty$. Therefore,

$$
\mathcal{H}_{\zeta}(A)=\lim _{\delta \rightarrow 0} \zeta_{\delta}(A)=\sup _{\delta>0} \zeta_{\delta}(A)
$$

defines the generalized $\zeta$-Hausdorff measure $\mathcal{H}_{\zeta}$.

In this construction, let $X$ be a complete Riemannian manifold $M$ and let $\mathcal{J}$ be the family of Borel subsets of $M$. Let $\Psi:[0, \infty) \rightarrow[0, \infty)$ a continuous function such that $\Psi(0)=0$. The $\Psi$-Hausdorff measure is defined by $\mathcal{H}_{\Psi}(A)=\mathcal{H}_{\zeta}(A)$ where $\zeta(A)=\Psi(\operatorname{diam}(A))$ and it is Borel regular, see [105, Thm. 4.2]. Taking $\mathcal{J}=\{$ open subsets of $M\}$ instead of the Borel sets and the same $\Psi$, the generalized Hausdorf measures obtained by the Carathéodory construction coincides, i.e they are the same $\Psi$-Hausdorff measure $\mathcal{H}_{\Psi}$, see [105, Thm. 4.4]. The choice $\Psi(t)=t^{\beta}$, for some fixed $\beta>0$, gives the standard $\beta$-dimensional Hausdorff measure $\mathcal{H}_{t^{\beta}}=\mathcal{H}^{\beta}$.

Remark 5.10. If $\mathcal{J}$ is the family of geodesic balls of $M$, the resulting measure $\overline{\mathcal{H}}_{\Psi}$ does not coincide, in general, with generalized Hausdorff measure $\mathcal{H}_{\Psi}$, see [105, Chap. 5]. However, if for some constant $c>0$ the following inequality holds $\Psi(2 t) \leq c \cdot \Psi(t)$, then $\mathcal{H}_{\Psi} \leq \overline{\mathcal{H}}_{\Psi} \leq c \mathcal{H}_{\Psi}$.

The first inequality $\mathcal{H}_{\Psi} \leq \overline{\mathcal{H}}_{\Psi}$ is obvious from the definition. To prove $\overline{\mathcal{H}}_{\Psi} \leq c \mathcal{H}_{\Psi}$ we proceed as follows. Since every open set $E_{j}$ is contained in a ball $B_{r_{j}}^{M}\left(x_{j}\right)$ of radius $r_{j}=\operatorname{diam}\left(E_{j}\right)$, we have that for every covering $\left\{E_{j}\right\}$ of $A \subseteq M$ with $\operatorname{diam}\left(E_{j}\right)<\delta$ that

$$
\sum_{i=1}^{+\infty} \Psi\left(\operatorname{diam}\left(E_{j}\right)\right) \geq \frac{1}{c} \cdot \sum_{i=1}^{+\infty} \Psi\left(2 \operatorname{diam}\left(E_{j}\right)\right)=\frac{1}{c} \cdot \sum_{i=1}^{+\infty} \Psi\left(\operatorname{diam}\left(B_{r_{j}}^{M}\left(x_{j}\right)\right)\right) .
$$

Taking the infimum, in the right hand-side, with respect to all covering $\left\{B_{r_{j}}^{M}\left(x_{j}\right)\right\}$ by balls of diameter less than $2 \delta$ and taking the infimum in the left hand side with respect of $E_{i}$ we have $\bar{\zeta}_{\delta} \leq c \cdot \zeta_{\delta},\left(\zeta=\Psi\right.$ (diam). Letting $\delta \downarrow 0$ we obtain the desired $\overline{\mathcal{H}}_{\Psi} \leq c \mathcal{H}_{\Psi}$.

5.1.3. Strategy of proof of Theorem 5.6. Let $M$ be a Riemannian manifold. To show that $-\Delta$ has discrete spectrum we rely on the well known characterization (84) of the essential spectrum, see [53], [114, Thm. 2.1], and Barta's eigenvalue lower bound, see [13], [22]. This characterization relates the infimum inf $\sigma_{\text {ess }}(-\Delta)$ of the essential spectrum of $-\Delta$ to the fundamental tone of the complements of compact sets. This is,

$$
\inf \sigma_{\text {ess }}(-\Delta)=\sup _{K \subset M} \lambda^{*}(M \backslash K),
$$

where $K$ is compact and $\lambda^{*}(M \backslash K)$ is the bottom of the spectrum of the Friedrichs extension of $\left(-\Delta, C_{c}^{\infty}(M \backslash K)\right)$, given by

$$
\lambda^{*}(M \backslash K)=\inf \left\{\frac{\int_{M \backslash K}|\nabla u|^{2}}{\int_{M \backslash K} u^{2}}, 0 \neq u \in C_{0}^{\infty}(M \backslash K)\right\} .
$$

On the other hand, Barta inequality gives a lower bound for $\lambda^{*}(M \backslash K)$ via positive functions, this is

$$
\lambda^{*}(M \backslash K) \geq \inf _{M \backslash K} \frac{-\Delta w}{w} \quad \text { for every } 0<w \in C^{2}(M \backslash K) .
$$


To prove that $-\Delta$ has discrete spectrum or equivalently, to prove that $\inf \sigma_{\text {ess }}(-\Delta)=$ $+\infty$, it is enough to find, for each small $\epsilon>0$, a compact set $K_{\epsilon} \subset M$ and a function $0<w_{\epsilon} \in C^{2}\left(M \backslash K_{\epsilon}\right)$ such that

$$
\frac{-\Delta w_{\epsilon}}{w_{\epsilon}} \geq c(\epsilon) \text { on } M \backslash K_{\epsilon},
$$

where $c(\epsilon) \rightarrow+\infty$ as $\epsilon \rightarrow 0$. Each $w_{\epsilon}$ will be constructed as a sum of suitable strictly positive superharmonic functions, depending on a good covering of $\lim \varphi$ by balls.

5.1.4. Main lemma. Let $\varphi: M \rightarrow N$ be an isometric immersion of a complete Riemannian $m$-manifold $M$ into a Riemannian $n$-manifold $N$, with mean curvature vector $H$. Let us suppose that $\varphi(M) \subset \Omega$, is a bounded, totally regular subset and let $b=\sup \left\{K_{N}(z), z \in\right.$ $\left.T_{\operatorname{diam}(\Omega)}(\Omega)\right\}$. Fix $\bar{a}>0$ such that $(\log (\bar{a}))^{2}>\log (\operatorname{diam}(\Omega))$ and if $b>0$, suppose in addition that $\bar{a} \leq \min \{\pi / 3 \sqrt{b}, \pi / 2(1+\theta) \sqrt{b}\}$. Recalling that

$$
\theta=\left[m-1-m \cdot \mu_{b}(\operatorname{diam}(\Omega)) \cdot\|H\|_{L^{\infty}(M)}\right]
$$

we have the following lemma.

Lemma 5.11 (Main Lemma). For each $a \in(0, \bar{a} / 3]$ and $x \in \Omega$ so that $\varphi(M) \subset B_{\operatorname{diam}(\Omega)}^{N}(x)$ if $\theta>0$ there exists $u \in C^{\infty}(M)$ satisfying these three conditions.

i. $u \geq 0$ and $u(p)=0$ if and only if $\varphi(p)=x$.

ii. $\Delta u \geq \theta / 3$ on $\varphi^{-1}\left(B_{a}^{N}(x)\right)$ if $\varphi^{-1}\left(B_{a}^{N}(x)\right) \neq \emptyset$.

iii. $\Delta u \geq 0$ on $M$.

iv.

$$
\|u\|_{L^{\infty}}(M) \leq \begin{cases}C a^{2}, & \text { if } \quad \theta>1 \\ C a^{2}|\log a|, & \text { if } \quad \theta=1 \\ C a^{\theta+1}, & \text { if } \quad 0<\theta<1,\end{cases}
$$

where $C$ is a positive constant depending on $m$, $\operatorname{diam}(\Omega),\|H\|_{L^{\infty}(M)}$.

Proof. Fix $x \in \Omega$ such that $\varphi(M) \subset B_{\operatorname{diam}(\Omega)}^{N}(x) \subset B_{r_{\Omega}}^{N}(x)$. Thus, the distance function $\rho(y)=\operatorname{dist}_{N}(x, y)$ is smooth (except at $y=x$ ) and the geodesic ball $B_{\operatorname{diam}(\Omega)}^{N}(x)$ is 1convex. In fact, by the hessian comparison theorem, [69],

$$
\bar{\nabla} \mathrm{d} \rho \geq \frac{h^{\prime}(\rho)}{h(\rho)}(\langle,\rangle-\mathrm{d} \rho \otimes \mathrm{d} \rho) .
$$

where $h:[0, \infty) \rightarrow[0, \infty)$ given by

$$
h(t)=\left\{\begin{array}{ccc}
\frac{1}{\sqrt{b}} \sin (\sqrt{b} t), & \text { if } \quad b>0 \\
t, & \text { if } \quad b=0 \\
\frac{1}{\sqrt{-b}} \sinh (\sqrt{-b} t), & \text { if } \quad b<0 .
\end{array}\right.
$$

Let $f \in C^{2}(N)$ be defined by $f(y)=g(\rho(y))$ for some $g \in C^{2}\left(\mathbb{R}_{0}^{+}\right)$that will be chosen later. The chain rule applied to the composition $f \circ \varphi \in C^{2}(M)$ implies that

$$
\nabla \mathrm{d}(f \circ \varphi)=\bar{\nabla} \mathrm{d} f(\mathrm{~d} \varphi, \mathrm{d} \varphi)+\mathrm{d} f\left(\bar{\nabla} \mathrm{d} \varphi^{\perp}\right) .
$$

where $\nabla, \bar{\nabla}$ are the connections of $M$ and $N$ respectively and $\bar{\nabla} \mathrm{d} \varphi^{\perp}$ is the second fundamental form of the immersion. Let $\left\{e_{i}, e_{\alpha}\right\}$ be a local Darboux frame along $\varphi$, with $\left\{e_{i}\right\}$ 
tangent to $M$. Tracing the above equality, it yields

$$
\Delta(f \circ \varphi)=\sum_{j=1}^{m} \bar{\nabla} \mathrm{d} f\left(e_{j}, e_{j}\right)+m \mathrm{~d} f(H) .
$$

On the other hand

$$
\bar{\nabla} \mathrm{d} f=g^{\prime \prime}(\rho) \mathrm{d} \rho \otimes \mathrm{d} \rho+g^{\prime}(\rho) \bar{\nabla} \mathrm{d} \rho .
$$

If $g^{\prime} \geq 0$ and by (87)

$$
\bar{\nabla} \mathrm{d} f \geq \frac{g^{\prime}(\rho) h^{\prime}(\rho)}{h(\rho)}(\langle,\rangle-\mathrm{d} \rho \otimes \mathrm{d} \rho)+g^{\prime \prime}(\rho) \mathrm{d} \rho \otimes \mathrm{d} \rho .
$$

Using $|\mathrm{d} \rho|=1$ and by (89)

$$
\begin{aligned}
\sum_{j=1}^{m} \bar{\nabla} \mathrm{d} f\left(e_{j}, e_{j}\right)+m \mathrm{~d} f(H)= & \frac{g^{\prime} h^{\prime}}{h}\left(m-\sum_{j=1}^{m} \mathrm{~d} \rho\left(e_{j}\right)^{2}\right)+g^{\prime \prime} \sum_{j=1}^{m} \mathrm{~d} \rho\left(e_{j}\right)^{2} \\
& +m g^{\prime} \mathrm{d} \rho(H) \\
\geq & \frac{g^{\prime} h^{\prime}}{h}\left(m-\sum_{j=1}^{m} \mathrm{~d} \rho\left(e_{j}\right)^{2}-m \frac{h}{h^{\prime}}\|H\|\right) \\
& +g^{\prime \prime} \sum_{j=1}^{m} \mathrm{~d} \rho\left(e_{j}\right)^{2} \\
\geq & \frac{g^{\prime} h^{\prime}}{h}(\overbrace{m-1-m \mu_{b}(\operatorname{diam}(\Omega))\|H\|_{L^{\infty}(M)}}) \\
& +g^{\prime \prime} \sum_{j=1}^{m} \mathrm{~d} \rho\left(e_{j}\right)^{2} . \\
= & \frac{g^{\prime} h^{\prime}}{h} \theta+g^{\prime \prime} \sum_{j=1}^{m} \mathrm{~d} \rho\left(e_{j}\right)^{2} .
\end{aligned}
$$

In other words,

$$
\Delta(f \circ \varphi) \geq \frac{g^{\prime} h^{\prime}}{h} \theta+g^{\prime \prime} \sum_{j=1}^{m} \mathrm{~d} \rho\left(e_{j}\right)^{2}
$$

Define $\omega:[0, \infty) \rightarrow \mathbb{R}$ by

$$
\omega(t)= \begin{cases}\left(1-\frac{t}{3 a(1+\theta)}\right)(\theta+1) h^{\prime}(t), & \text { if } t \leq 3 a(1+\theta) \\ 0, & \text { if } t \geq 3 a(1+\theta),\end{cases}
$$

where $3 a \leq \bar{a}$. Setting

$$
g(t)=\int_{0}^{t} \frac{1}{h(s)^{\theta}}\left[\int_{0}^{s} h(\sigma)^{\theta} \omega(\sigma) \mathrm{d} \sigma\right] \mathrm{d} s .
$$

We have that $g$ is solution of

$$
g^{\prime}(t) \frac{h^{\prime}(t)}{h(t)} \theta+g^{\prime \prime}(t)=\omega(t)
$$

It is easy to show that $g \in C^{2}([0, \infty))$. From (93) we have that if $t \leq 3 a(1+\theta)$, then 


$$
\begin{aligned}
g^{\prime \prime}(t) & =\omega(t)-\frac{\theta h^{\prime}(t)}{h(t)^{1+\theta}(t)} \int_{0}^{t}\left(1-\frac{s}{3 a(1+\theta)}\right) \frac{\mathrm{d}}{\mathrm{d} s}\left(h^{1+\theta}(s)\right) \mathrm{d} s \\
& =\omega(t)-\theta h^{\prime}(t)+\frac{\theta h^{\prime}(t)}{h^{(\theta+1)}(t)} \int_{0}^{t} \frac{s}{3 a} h^{\theta}(s) h^{\prime}(s) \mathrm{d} s \\
& =\left(1-\frac{t}{3 a}\right) h^{\prime}(t)+\frac{\theta h^{\prime}(t)}{h^{(\theta+1)}(t)} \int_{0}^{t} \frac{s}{3 a} h^{\theta}(s) h^{\prime}(s) \mathrm{d} s
\end{aligned}
$$

From (94) we have that $g^{\prime \prime}(t) \geq 0$ if $t \leq 3 a$. Moreover, $h^{\prime}(t) \geq 1 / 2$ if $t \leq 3 a$. Then at any $x^{\prime} \in \varphi^{-1}\left(B_{a}^{N}(x)\right)$ we have from $(91)$

$$
\begin{aligned}
\Delta f \circ \varphi\left(x^{\prime}\right) & \geq \frac{g^{\prime} h^{\prime}}{h} \theta+g^{\prime \prime} \sum_{j=1}^{m} \mathrm{~d} \rho\left(e_{j}\right)^{2} . \\
& \geq g^{\prime}(\rho(\varphi(x))) \frac{h^{\prime}}{h} \rho(\varphi(x)) \theta, \\
& \geq\left(1-\frac{\rho(\varphi(x))}{3 a(1+\theta)}\right) \theta h^{\prime}(\rho(\varphi(x))) \\
& \geq \frac{1}{2}\left(1-\frac{\rho(\varphi(x))}{3 a(1+\theta)}\right) \theta \\
& \geq \frac{\theta}{3} .
\end{aligned}
$$

Decompose

$$
M=\left\{y \in M: g^{\prime \prime}(\rho(\varphi(y))) \geq 0\right\} \cup\left\{y \in M: g^{\prime \prime}(\rho(\varphi(y)))<0\right\}=A \cup B .
$$

We have that inequality (95) shows that if $x^{\prime} \in A$ then $\Delta f \circ \varphi(x) \geq 0$. On the other hand, at any point $x^{\prime} \in B$ we have by (91) and by the fact that

$$
|\bar{\nabla} \rho|^{2}=1=\sum_{j=1}^{m} \mathrm{~d} \rho\left(e_{j}\right)^{2}+\sum_{\alpha=m+1}^{n} \mathrm{~d} \rho\left(e_{\alpha}\right)^{2} \geq \sum_{j=1}^{m} \mathrm{~d} \rho\left(e_{j}\right)^{2},
$$

that

$$
\begin{aligned}
\Delta f \circ \varphi(x) & \geq\left[g^{\prime} \frac{h^{\prime}}{h} \theta+g^{\prime \prime} \sum_{j=1}^{m} \mathrm{~d} \rho\left(e_{j}\right)^{2}\right], \\
& \geq g^{\prime} \frac{h^{\prime}}{h} \theta+g^{\prime \prime} \\
& \geq \omega \\
& \geq 0 .
\end{aligned}
$$

Observe that

$$
\int_{0}^{t} h(s)^{\theta} \omega(s) \mathrm{d} s \leq\left\{\begin{array}{lll}
h(t)^{1+\theta}, & \text { if } & 0 \leq t \leq 3 a(1+\theta) \\
h\left(t_{1}\right)^{1+\theta}, & \text { if } & t>t_{1}=3 a(1+\theta) .
\end{array}\right.
$$

Taking in account that $c_{1} \cdot t \leq h(t) \leq c_{2} \cdot t, t \in[0, \operatorname{diam}(\Omega)]$ for some positive constants $c_{1}, c_{2}$, we have the following upper bounds for $g$. 
If $0 \leq t \leq t_{1}=3 a(1+\theta)$,

$$
\begin{aligned}
g(t) & =\int_{0}^{t} \frac{1}{h(s)^{\theta}}\left[\int_{0}^{s} h(\sigma)^{\theta} \omega(\sigma) \mathrm{d} \sigma\right] \mathrm{d} s \\
& \leq \int_{0}^{t} h(s) \mathrm{d} s . \\
& \leq c_{2} \frac{\left(t_{1}\right)^{2}}{2}=9 \cdot c_{2} \cdot \frac{(1+\theta)^{2}}{2} \cdot a^{2} .
\end{aligned}
$$

If $t \geq t_{1}=3 a(1+\theta)$,

$$
\begin{aligned}
& g(t)=\int_{0}^{a} \frac{1}{h(s)^{\theta}}\left[\int_{0}^{s} h(\sigma)^{\theta} \omega(\sigma) \mathrm{d} \sigma\right] \mathrm{d} s+\int_{a}^{t} \frac{1}{h(s)^{\theta}}\left[\int_{0}^{t_{1}} h(\sigma)^{\theta} \omega(\sigma) \mathrm{d} \sigma\right] \mathrm{d} s \\
& \leq \int_{0}^{a} h(s) \mathrm{d} s+h^{1+\theta}\left(t_{1}\right) \int_{a}^{t} \frac{1}{h(s)^{\theta}} \mathrm{d} s \\
& \leq \frac{c_{2}}{2} \cdot a^{2}+\frac{c_{2}^{(1+\theta)}(3 a(1+\theta))^{(1+\theta)}}{c_{1}} \int_{a}^{t} \frac{1}{s^{\theta}} \mathrm{d} s \\
& =c_{3} \cdot a^{2}+c_{4} \cdot a^{(\theta+1)} \int_{a}^{t} \frac{1}{s^{\theta}} \mathrm{d} s \\
& \leq c_{3} \cdot a^{2}+c_{4} \cdot a^{(\theta+1)} \begin{cases}\frac{a^{1-\theta}}{\theta-1}, & \text { if } \theta>1 \\
c_{5} \cdot|\ln a|, & \text { if } \theta=1 \\
\frac{t^{1-\theta}}{1-\theta} \leq \frac{\operatorname{diam}(\Omega)^{1-\theta}}{1-\theta}, & \text { if } \quad 0<\theta<1 .\end{cases}
\end{aligned}
$$

We can deduce from (98) and (99) that there exists a positive constant $C$ depending on $m, \operatorname{diam}(\Omega), b$ and $\|H\|_{L^{\infty}(M)}$ such that

$$
\|g\|_{L^{\infty}([0, \operatorname{diam}(\Omega)])} \leq \begin{cases}C a^{2}, & \text { if } \theta>1 \\ C a^{2}|\log a|, & \text { if } \theta=1 \\ C a^{\theta+1}, & \text { if } \theta \in(0,1) .\end{cases}
$$

Taking $u=f \circ \varphi: M^{m} \rightarrow \mathbb{R}$ we have that

- By construction $u(p)=0$ if and only if $\varphi(p)=x$.

- By (95) and (97) we have $\Delta u \geq \theta / 3$ on $\varphi^{-1}\left(B_{a}^{N}(x)\right)$ and $\Delta u \geq 0$ on $M$, respectively.

- By (99) we have

$$
\|u\|_{L^{\infty}(M)} \leq\|f\|_{L^{\infty}\left(\varphi^{-1}\left(B_{\operatorname{diam}(\Omega)}^{N}(x)\right)\right)}=\|g\|_{L^{\infty}([0, \operatorname{diam}(\Omega)])} .
$$

This proves Lemma 5.11 .

5.1.5. Strictly $m$-convex domains. A strictly $m$-convex domain $\Omega$ with constant $c>0$ is related to the existence of strictly $m$-subharmonic functions on $\Omega$.

Definition 5.12. A $C^{2}$-function $\phi: \Omega \rightarrow \mathbb{R}$ is said to be strictly $m$-subharmonic with constant $c>0$ if $\lambda_{1}(p) \leq \lambda_{2}(p) \leq \cdots \leq \lambda_{n}(p)$ are the ordered eigenvalues of the hessian 
$\bar{\nabla} \mathrm{d} \phi(p)$, then there exists an $\epsilon>0$ such that

$$
\left\{\begin{array}{l}
\lambda_{1}(p)+\cdots+\lambda_{m}(p) \geq c, \forall p \in T_{\epsilon}^{N}(\partial \Omega)=\left\{y \in N: \operatorname{dist}_{N}(y, \partial \Omega) \leq \epsilon\right\} \\
\lambda_{1}(p)+\cdots+\lambda_{m}(p) \geq 0, \forall p \in \Omega .
\end{array}\right.
$$

Let $\Omega \subset N$ be a strictly $m$-convex domain of $N$ with constant $c>0$ and $\Gamma=\partial \Omega$ of class $C^{3}$. Let $t: N \rightarrow \mathbb{R}$ be the oriented distance function to $\Gamma$ with orientation outward $\Omega$. This is,

$$
t(y)=\left\{\begin{aligned}
-\operatorname{dist}_{N}(y, \partial \Omega), & \text { if } \quad y \in \Omega \\
\operatorname{dist}_{N}(y, \partial \Omega), & \text { if } \quad y \in N \backslash \Omega .
\end{aligned}\right.
$$

The oriented distance $t(y)$ is Lipschitz in $N$ and of class $C^{2}$ in a tubular neighborhood $T_{\epsilon_{0}}^{N}(\partial \Omega)$ for some $\epsilon_{0}$. Let $\alpha_{s}$ be the shape operator of the parallel hypersurface $\Gamma_{s}=t^{-1}(s)$, $|s| \leq \epsilon_{0}$ with respect to the normal vector field $-\bar{\nabla} t$. At each point of $\Gamma_{s}$ there is an orthonormal bases of $T \Gamma_{s}$ such that $\alpha_{s}$ is diagonalized

$$
\alpha_{s}=\operatorname{diag}\left(\xi_{1}^{s}, \xi_{2}^{s}, \ldots, \xi_{n-1}^{s}\right),
$$

where $\xi_{1}^{s} \leq \xi_{2}^{s} \leq \ldots \leq \xi_{n-1}^{s}$. By the uniform continuity of each $\xi_{j}^{s}$ and the compactness of $\overline{T_{\epsilon_{0}}^{N}(\partial \Omega)}$, for each $\delta \in(0,1)$ one can choose $\epsilon_{0}$ small enough to have

$$
\xi_{1}^{s}(y)+\cdots+\xi_{m}^{s}(y) \geq \delta c
$$

$\forall y \in T_{\epsilon_{0}}^{N}(\partial \Omega)$. Let $\epsilon_{1}$ be a positive number so that

$$
\epsilon_{1}<\min \left\{1, \epsilon_{0},\left\|\alpha_{s}\right\|_{L^{\infty}\left(T_{\epsilon_{0}}^{N}(\partial \Omega)\right)}^{-1}\right\}
$$

Define $\Phi_{\epsilon}: N \rightarrow \mathbb{R}, 0<\epsilon<\epsilon_{1} / 2$, by

$$
\Phi_{\epsilon}(y)= \begin{cases}-2 \epsilon, & \text { if } \quad t(y) \leq-2 \epsilon \\ 2 \epsilon\left[\left(\frac{t(y)}{2 \epsilon}+1\right)^{3}-1\right], & \text { if } \quad t(y) \geq-2 \epsilon .\end{cases}
$$

The function $\Phi_{\epsilon}$ is Lipschitz in $N$ and $C^{2}$ in the tubular neighborhood $\overline{T_{\epsilon_{0}}^{N}(\Omega)}=t^{-1}\left(\left(-\infty, \epsilon_{0}\right]\right)$. For $t(y) \leq \epsilon_{0}$, we can compute the gradient and the hessian of $\Phi_{\epsilon}$ as follows.

$$
\begin{aligned}
& \bar{\nabla} \Phi_{\epsilon}(y)= \begin{cases}0, & \text { if } t(y) \leq-2 \epsilon \\
3\left(\frac{t(y)}{2 \epsilon}+1\right)^{2} \bar{\nabla} t(y), & \text { if } \quad-2 \epsilon \leq t(y) \leq \epsilon_{0} .\end{cases} \\
& \bar{\nabla} \mathrm{d} \Phi_{\epsilon}(y)(X, Y)=\left\{\begin{array}{llr}
0, & \text { if } & t(y) \leq-2 \epsilon \\
3\left(\frac{t(y)}{2 \epsilon}+1\right)^{2} \bar{\nabla} \mathrm{d} t(y)(X, Y), & \\
+\frac{3}{\epsilon}\left(\frac{t(y)}{2 \epsilon}+1\right) X(t) Y(t) . & \text { if } & -2 \epsilon \leq t(y) \leq \epsilon_{0}
\end{array}\right.
\end{aligned}
$$

Writing $\bar{\nabla} \mathrm{d} \Phi_{\epsilon}(y)(X, Y)=\langle S(X), Y\rangle$, for an appropriate symmetric endomorphism $S: T N \rightarrow T N$, we have that for $-2 \epsilon \leq t(y) \leq 2 \epsilon, S(y)$ can be represented by a diagonal 
matrix,

$$
S(y)=\operatorname{diag}\left(3\left(\frac{t(y)}{2 \epsilon}+1\right)^{2} \xi_{1}^{t}(y), \ldots, 3\left(\frac{t(y)}{2 \epsilon}+1\right)^{2} \xi_{n-1}^{t}(y), \frac{3}{\epsilon}\left(\frac{t(y)}{2 \epsilon}+1\right)\right)
$$

Since

$$
\begin{aligned}
3\left(\frac{t(y)}{2 \epsilon}+1\right)^{2} \xi_{j}^{t}(y)-\frac{3}{\epsilon}\left(\frac{t(y)}{2 \epsilon}+1\right) & =3\left(\frac{t(y)}{2 \epsilon}+1\right)\left[\left(\frac{t(y)}{2 \epsilon}+1\right) \xi_{j}^{t}(y)-\frac{1}{\epsilon}\right] \\
& \leq 6\left[2 \xi_{j}^{t}-\frac{1}{\epsilon_{1}}\right] \\
& \leq 12\left(\xi_{j}^{t}(y)-2\left\|\alpha_{t}\right\|_{L^{\infty}\left(T_{\epsilon_{0}}^{N}(\partial \Omega)\right)}\right) \\
& \leq 0 .
\end{aligned}
$$

We obtain $\lambda_{1} \leq \lambda_{2} \leq \cdots \leq \lambda_{n}, \lambda_{j}=3\left(\frac{t}{2 \epsilon}+1\right) \xi_{j}^{t}, j=1, \ldots n-1, \lambda_{n}(y)=\frac{3}{\epsilon}\left(\frac{t(y)}{2 \epsilon}+1\right)$ with $S=\operatorname{diag}\left(\lambda_{1}, \lambda_{2}, \ldots \lambda_{n}\right)$. By Lemma 2.3 of [83], we have that for any subspace $V \subset T_{y} N, y \in T_{2 \epsilon}^{N}(\partial \Omega)$ and $1 \leq \operatorname{dim} V=m \leq n-1$ that

$$
\begin{aligned}
\operatorname{Trace}\left(\bar{\nabla} \mathrm{d} \Phi_{\epsilon}(y) \mid V\right) & \geq \lambda_{1}(y)+\cdots+\lambda_{m}(y) \\
& \geq 3\left(\frac{t(y)}{2 \epsilon}+1\right)^{2}\left[\xi_{1}^{t(y)}+\cdots+\xi_{m}^{t(y)}\right] \\
& \geq 3\left(\frac{t(y)}{2 \epsilon}+1\right)^{2} \delta c .
\end{aligned}
$$

Then

- If $t(y) \leq 2 \epsilon$, we obtain that, $\operatorname{Trace}\left(\bar{\nabla} \mathrm{d} \Phi_{\epsilon}(y) \mid V\right) \geq 0$ and

- for $|t(y)| \leq \epsilon(1-\sqrt{\delta})$, we obtain,

$$
\operatorname{Trace}\left(\bar{\nabla} \mathrm{d} \Phi_{\epsilon}(y) \mid V\right) \geq 3(1+\sqrt{\delta})^{2} \delta c / 4 \text {. }
$$

This proves the following lemma.

Lemma 5.13. Let $\Omega$ be a strictly $m$-convex, $1 \leq m \leq n-1$, with constant $c>0$. There exists a Lipschitz function $\Phi_{\epsilon}: N \rightarrow \mathbb{R}$, that is $C^{2}$ in $T_{2 \epsilon}(\Omega)$, where $2 \epsilon<\epsilon_{1}, \epsilon_{1}$ is a positive number depending on the geometry of $\partial \Omega$, see (101), and such that

1. $\Phi_{\epsilon}^{-1}((-\infty, 0))=\Omega, \Phi_{\epsilon}^{-1}(0)=\partial \Omega$.

2. $\left|\Phi_{\epsilon}\right| \leq 2 \epsilon$ in $\bar{\Omega}$.

3. Trace $\left(\bar{\nabla} \mathrm{d} \Phi_{\epsilon}(y) \mid V\right) \geq 3(1+\sqrt{\delta})^{2} \delta c / 4$, for $|t(y)| \leq \epsilon(1-\sqrt{\delta})$ and any m-subspace $V \subset T_{y} N$.

4. Trace $\left(\bar{\nabla} \mathrm{d} \Phi_{\epsilon}(y) \mid V\right) \geq 0$, in $\Omega$ for any m-subspace $V \subset T_{y} N$.

In other words, $\Phi_{\epsilon}$ is strictly $m$-subharmonic function with constant $3(1+\sqrt{\delta})^{2} \delta c / 4$.

We will need the following lemma for the proof of Theorem 5.6.

Lemma 5.14. Let $\varphi: M^{m} \rightarrow N^{n}$ be an isometric immersion such that there exists a bounded, totally regular, strictly $m$-convex domain $\Omega \subset N$ with constant $c>0$ and $C^{3}$ boundary $\partial \Omega$ such that $\varphi(M) \subset \Omega, \mathcal{H}_{\Psi}(\lim \varphi \cap \Omega)=0$ and

$$
\|H\|_{L^{\infty}(M)}<\min \left\{\frac{m-1}{m \cdot \mu_{b}(\operatorname{diam}(\Omega))}, \frac{c}{m}\right\} .
$$

Take $\delta \in(0,1)$ such that

$$
\|H\|_{L^{\infty}}(M)<\frac{\delta^{2} c}{m}
$$


and let $\epsilon<\epsilon_{1} / 2$ as above in Lemma 5.13. Then the function $u: M^{m} \rightarrow \mathbb{R}$ given by $u=\Phi_{\epsilon} \circ \varphi$, where $\Phi_{\epsilon}$ is also given in Lemma 5.13, satisfies

(1) $|u(x)| \leq 2 \epsilon$ for all $x \in M$.

(2) $\Delta u(x) \geq 0$ for all $x \in M$.

(3) $\Delta u(x) \geq C_{\delta}$, if $|t(\varphi(x))| \leq \epsilon(1-\sqrt{\delta})$, where $C_{\delta}=3 c \cdot \delta \cdot(1-\delta) \cdot(1+\sqrt{\delta})^{2} / 4$.

(4) $\varphi(M) \cap \partial \Omega=\emptyset$.

Proof. Taking $u=\Phi_{\epsilon} \circ \varphi$ the item 1. holds by the item 2. of Lemma 5.13 and the fact that $\varphi(M) \subset \Omega$. On the other hand, we have by (103)

$$
\begin{aligned}
\Delta u(x) & =\operatorname{Trace}\left(\bar{\nabla} \mathrm{d} \Phi_{\epsilon} \mid T_{\varphi(x)} M\right)+<\nabla \Phi_{\epsilon}, m H> \\
& \geq 3\left(\frac{t(\varphi(x))}{2 \epsilon}+1\right)^{2} \delta c-3\left(\frac{t(\varphi(x))}{2 \epsilon}+1\right)^{2} \delta^{2} c \\
& =3\left(\frac{t(\varphi(x))}{2 \epsilon}+1\right)^{2} \delta c(1-\delta) \\
& \geq 0 .
\end{aligned}
$$

This proves item 2. If $|t(\varphi(x))| \leq \epsilon(1-\sqrt{\delta})$ we get

$$
\Delta u(x) \geq \frac{3}{4}(1+\sqrt{\delta})^{2}(1-\delta) \delta c
$$

and that proves item 3. If there exists a $x \in \varphi^{-1}(\varphi(M) \cap \partial \Omega)$ then $\Delta u(x)>0$ by (105). On the other hand $u$ has a maximum at $x$ therefore $\Delta u(x) \leq 0$ a contradiction. This proves item 4 and finishes the proof of Lemma 5.14.

5.1.6. End of the proof of Theorem 5.6. Theorem 5.6 states that if $\varphi: M \rightarrow N$ is an isometric immersion of a Riemannian $m$-manifold $M$ into a Riemannian $n$-manifold $N$ with mean curvature vector $H$ such that $\varphi(M) \subset \Omega$, is a bounded, totally regular, open subset of $N$ and let $b$ be as in (78) and $\mu_{b}$ as defined in (79). Assume that

$$
\|H\|_{L^{\infty}(M)}<\frac{m-1}{m \cdot \mu_{b}(\operatorname{diam}(\Omega))} .
$$

Define $\theta=\left[m-1-m \cdot \mu_{b}(\operatorname{diam}(\Omega)) \cdot\|H\|_{L^{\infty}(M)}\right]>0$ and $\Psi \in C^{0}([0, \infty)$ given by

$$
\Psi(t)=\left\{\begin{array}{lll}
t^{2}, & \text { if } & \theta>1 \\
t^{2}|\log t|, & \text { if } & \theta=1 \\
t^{\theta+1}, & \text { if } & \theta \in(0,1) .
\end{array}\right.
$$

If one of the following conditions holds

(1) $\lim \varphi \cap \partial \Omega=\emptyset$ and $\mathcal{H}_{\Psi}(\lim \varphi)=0$,

(2) $\lim \varphi \cap \partial \Omega \neq \emptyset, \mathcal{H}_{\Psi}(\lim \varphi \cap \Omega)=0, \Omega$ is strictly $m$-convex with constant $c>0$, $\partial \Omega$ is of class $C^{3}$, and the mean curvature vector $H$ satisfies the further restriction

$$
\|H\|_{L^{\infty}(M)}<\frac{c}{m}
$$

then the spectrum of $-\Delta$ is discrete. To prove this result we proceed as follows.

Let $\varphi: M \rightarrow N$ be an isometric immersion of a Riemannian $m$-manifold $M$ into a Riemannian $n$-manifold $N$ with mean curvature vector $H$. Suppose that $\varphi(M) \subset \Omega$ for a bounded totally regular subset $\Omega$. Let $b=\sup \left\{K_{N}(z), z \in T_{\operatorname{diam}(\Omega)}(\Omega)\right\}$ and $\|H\|_{L^{\infty}(M)}<(m-1) / m \cdot \mu_{b}(\operatorname{diam}(\Omega))$. First we will prove Theorem 5.6 under the assumptions of item 1 . Suppose that $\mathcal{H}_{\Psi}(\lim \varphi)=0$. Choose a positive number $\bar{a}>0$ such that $(\log (\bar{a}))^{2}>\log (\operatorname{diam}(\Omega))$ and if $b>0$ take $\bar{a} \leq \min \{\pi / 3 \sqrt{b}, \pi / 2(1+\theta) \sqrt{b}\}$, where

$$
\theta=m-1-m \mu(\operatorname{diam}(\Omega))\|H\|_{L^{\infty}(M)} .
$$


Observe that $\Omega \subset B_{\operatorname{diam}(\Omega)}^{N}\left(x_{0}\right)$ for $x_{0} \in \Omega$. Then choose $r_{1} \ll \operatorname{diam}(\Omega)$ such that the $2 r_{1^{-}}$ tubular neighborhood $T_{2 r_{1}}(\lim \varphi) \subset B_{\operatorname{diam}(\Omega)}^{N}\left(x_{0}\right)$. Fix $\epsilon \in\left(0, r_{1}\right)$. Since $\mathcal{H}_{\Psi}(\lim \varphi)=0$ and Remark 5.10, there is $a>0$ and a countable covering of $\lim \varphi$ by geodesic balls $B_{j}=B_{a_{j}}^{N}\left(y_{j}\right) \subset N$ of radius $2 a_{j} \leq a \leq \min \left\{r_{1}, \bar{a} / 3\right\}$ such that

$$
\lim \varphi \subset \bigcup_{j} B_{j} \quad \text { and } \quad\left|\sum_{j} \Psi\left(2 a_{j}\right)\right|<\epsilon .
$$

Since $\lim \varphi$ is compact we can extract a finite sub-covering $\left\{B_{j}\right\}_{j=1}^{k}$ of $\lim \varphi$ such that (110) holds, and each $B_{j} \subset T_{2 r_{1}}(\lim \varphi)$ for all $j=1, \ldots, k$. Applying Lemma 5.11, we construct, for every $j=1, \ldots, k$, a function $u_{j}: M \rightarrow \mathbb{R}$ such that

$$
\left\{\begin{array}{l}
u_{j} \geq 0, \quad u_{j}(p)=0 \text { if and only if } \varphi(p)=y_{j}, \\
\left\|u_{j}\right\|_{L^{\infty}(M)} \leq C \Psi\left(2 a_{j}\right), \\
\Delta u_{j} \geq 0 \text { on } M, \quad \Delta u_{j} \geq \theta / 3 \text { on } \varphi^{-1}\left(B_{j}\right)
\end{array}\right.
$$

where $C$ is positive constant depending on $m, \operatorname{diam}(\Omega),\|H\|_{L^{\infty}(M)}$.

Let $w_{1}=\sum_{j=1}^{k_{1}}\left(2\left\|u_{j}\right\|_{L^{\infty}}-u_{j}\right)>0$. By the boundedness of $\varphi(M)$ the set

$$
K_{\epsilon}=M \backslash \varphi^{-1}\left(\bigcup_{j=1}^{k_{1}} B_{j}\right)
$$

is compact in $M$. Now, by $(2.21)$ the fundamental tone

$$
\lambda^{*}\left(M \backslash K_{\epsilon}\right) \geq \inf _{M \backslash K_{\epsilon}}\left(-\frac{\Delta_{M} w_{1}}{w_{1}}\right) .
$$

Let $q \in M \backslash K_{\epsilon}$ then $\varphi(q) \in \bigcup_{j=1}^{k_{1}} B_{j}$. Let $j^{\prime}$ be so that $\varphi(q) \in B_{j^{\prime}}$. Then $\Delta_{M} u_{j^{\prime}}(q) \geq$ $\theta / 3$ and $\Delta_{M} u_{j}(q) \geq 0$ for all other $j^{\prime} s$. Therefore,

$$
\begin{aligned}
-\frac{\Delta w_{1}}{w_{1}}(q) & \geq \frac{\sum_{j} \Delta_{M} u_{j}(q)}{2 \sum_{j}\left\|u_{j}\right\|_{L^{\infty}}} \\
& \geq \frac{\Delta_{M} u_{j^{\prime}}(q)}{2 C \sum_{j} \Psi\left(2 a_{j}\right)} \\
& \geq \frac{\theta}{6 C \epsilon} .
\end{aligned}
$$

Here $C=C\left(m, R_{1},\|H\|_{L^{\infty}(M)}\right)$. This shows that $\lambda^{*}\left(M \backslash K_{\epsilon}\right) \geq \frac{\theta}{6 C \epsilon}$ for each $\epsilon \in\left(0, r_{1}\right)$. Therefore $\lambda^{*}\left(M \backslash K_{\epsilon}\right) \rightarrow+\infty$ if $\epsilon \rightarrow 0$ and proves item 1 .

To prove item 2. we recall that we have an isometric immersion $\varphi: M^{m} \rightarrow N^{n}$ of a Riemannian manifold $M$ into a Riemannian manifold $N$ with mean curvature vector $H$ such that $\varphi(M) \subset \Omega$, a totally regular, strictly $m$-convex domain with constant $c>0$ and $C^{3}$-boundary $\partial \Omega$ and $\Psi$-Hausdorff measure $\mathcal{H}_{\Psi}(\lim \varphi \cap \Omega)=0$. The mean curvature vector is assumed to satisfy $\|H\|_{L^{\infty}(M)}<\min \left\{(m-1) / m \cdot \mu_{b}(\operatorname{diam}(\Omega)), c / m\right\}$. We may assume that $\lim \varphi \cap \partial \Omega \neq \emptyset$, otherwise we can apply item 1. By Lemma 5.14, there exist positive numbers $\delta=\delta(\varphi), C_{\delta}>0$ and $\epsilon_{1}=\epsilon_{1}(\Omega)$ such that for any $\epsilon<\epsilon_{1} / 2$, there exists a $C^{2}$ function $u: M \rightarrow \mathbb{R}$, such that

1. $\left.u^{-1}(-\infty, 0)\right)=M$.

2. $|u(x)| \leq 2 \epsilon$ in $M$.

3. $\Delta u(x) \geq 0$ for all $x \in M$. 
4. $\Delta u(x) \geq C_{\delta}$, if $\varphi(x) \in T_{\epsilon(1-\sqrt{\delta})}(\partial \Omega)$.

Fix one $\epsilon, 0<\epsilon<\epsilon_{1} / 2$ and set $K=\lim \varphi \backslash T_{\epsilon(1-\sqrt{\delta})}(\partial \Omega)$. We have $K \subset \lim \varphi \cap \Omega$ compact $\mathcal{H}_{\Psi}(K)=0$. By the first part of this proof we have finite functions $u_{j}: M \rightarrow \mathbb{R}$ and balls $B_{j} \subset \Omega$ (covering $\left.K\right)$ such that (110) and (111) holds. Take $w_{1}=\sum_{j=1}^{k_{1}}\left(2\left\|u_{j}\right\|_{L^{\infty}}-u_{j}\right)>0$ (related to $K$ ) and $u: M \rightarrow \mathbb{R}$ given by Lemma 5.14. Define $\omega: M \rightarrow \mathbb{R}$ by

$$
\omega(x)=\omega_{1}(x)+\epsilon-u(x), \quad x \in M
$$

and

$$
K_{\epsilon}=M \backslash \varphi^{-1}\left(\left(\cup_{j=1}^{k} B_{j}\right) \cup T_{\epsilon(1-\sqrt{\delta})}(\partial \Omega)\right)
$$

The set $K_{\epsilon}$ is compact and for $x \in M \backslash K_{\epsilon}$ we get

$$
-\Delta \omega \geq c_{0}=\min \left\{\frac{\theta}{3}, C_{\delta}\right\}>0 .
$$

Since $0<\omega(x)<(2 C+3) \epsilon, x \in M$, we get

$$
-\frac{\Delta \omega}{\omega} \geq \frac{c_{0}}{(2 C+3) \epsilon} .
$$

Then $\lambda^{*}\left(M \backslash K_{\epsilon}\right) \rightarrow \infty$ if $\epsilon \rightarrow 0$ what proves item 2 .

\section{Nonempty ESSENTIAL SPECTRUM}

As a counterpart of Theorem 5.6, in this section we study conditions under which the essential spectrum $\sigma_{\text {ess }}(M)$ is nonempty, and in particular when it contains (or coincides with) a half-line $[c,+\infty)$. The problem captured the attention of researchers since the seventies, and generated a vast literature which we barely touch in this survey. The importance of this investigation has been highlighted, for instance, in S.T. Yau's lectures [140]. We first made a brief account of the state of the art in the intrinsic case, and then we move to the case of immersed submanifolds, where we describe in detail our main contributions.

To begin with, we recall that the well-known Weyl's characterization for the spectrum implies the following

Lemma 6.1. [50, Lemma 4.1.2] A number $\lambda \in \mathbb{R}$ lies in $\sigma(M)$ if and only if there exists a sequence of nonzero functions $u_{j} \in \operatorname{Dom}(-\Delta)$ such that

$$
\left\|\Delta u_{j}+\lambda u_{j}\right\|_{2}=o\left(\left\|u_{j}\right\|_{2}\right) \quad \text { as } j \rightarrow+\infty .
$$

As already underlined, characterizations of the whole $\sigma(M)$ are known only in few special cases. Among them the space forms $\mathbb{M}_{\kappa}^{m}$ of curvature $-\kappa \leq 0$, for which

$$
\sigma\left(\mathbb{M}_{\kappa}^{m}\right)=\left[\frac{(m-1)^{2} \kappa}{4},+\infty\right) .
$$

Hereafter, given a complete Riemannian manifold $M^{m}$, with $\rho(x)$ we denote the distance of $x$ from a fixed reference origin $o \in M$. The approach to guarantee that $\sigma(M)=[c,+\infty)$, for some $c \geq 0$, usually splits into two parts.

1) The first one is to show that $\inf \sigma(M) \geq c$ and, if $c>0$, it requires in general binding conditions such as those guaranteeing the Laplacian comparison from below for $\Delta \rho$. In particular, it requires $o$ to be a pole for $M$. For instance, see [104], [22].

2) The second one is to produce a sequence of approximating eigenfunctions like in lemma 6.1 for each $\lambda>c$. This step is accomplished by considering radial functions of compact support, and, at least in the first results on the topic like the one in [52], uses the comparison theorems on both sides for $\Delta \rho$. Therefore, the method 
needs both a pinching on the sectional curvature and, again, the smoothness of $\rho$, that is, that $o$ is a pole of $M$ (see [52], [62], [141], [90] and Corollary 2.17 in [26]). The prototype example of the above technique is H. Donnelly's result in [52], which states that $\sigma\left(M^{m}\right) \equiv \sigma\left(\mathbb{N}_{\kappa}^{m}\right)$ provided that $o$ is a pole and $K_{\text {rad }}(x) \rightarrow \kappa \leq 0$ as $\rho(x) \rightarrow+\infty$.

Taking into account that the pole requirement is a severe restriction, since then various efforts were made to weaken both the curvature and the topological assumptions, and especially criteria to guarantee that a half-line $[c,+\infty)$ belongs to $\sigma(M)$ (actually, to $\left.\sigma_{\text {ess }}(M)\right)$ have been extensively investigated. We remark that the decomposition principle by Donnelly-Li [53] states that the essential spectrum just depends on the geometry of $M$ outside a compact set, thus 2) is expected to require just conditions at infinity on $M$. For instance, as observed in $[88,89]$ the need for a pole can be replaced by the existence of a relatively compact, mean convex, smooth open set $\Omega$ such that the normal exponential map realizes a global diffeomorphism $\partial \Omega \times \mathbb{R}_{0}^{+} \rightarrow M \backslash \Omega$. Conditions of this kind seem, however, unavoidable for the the techniques in the aforementioned papers to work. However, curvature assumptions to establish Step 2 can be drastically weakened: in [89], H. Kumura was able to replace the two-sided pinching on the sectional curvature with a combination of a lower bound on a suitably weighted volume and an $L^{p}$-bound on the Ricci curvature.

From another perspective, in [106] the authors considered a class of complete manifolds where an assumption like Donnelly's one in [52] is given just in a non-compact "drop-like" region $\Omega \subset M$. Under suitable conditions on the metric and on the spike of the drop, they showed that $\sigma_{\mathrm{ess}}(M)$ contains a half-line $[c,+\infty)$, for an explicit $c$. This applies to show that, for instance, a horoball $\Omega \subset \mathbb{H}^{n}$ has the same spectrum ${ }^{6}$ of the whole $\mathbb{H}^{m}$.

When the distance function (from a point, or from $\partial \Omega$ ) is not smooth, radial functions in general cannot belong to $\operatorname{dom}(-\Delta)$. However, one can produce "radial" eigenfunctions of the form $u_{j}=\psi_{j}(\gamma)$ provided that there exists an exhaustion $\gamma \in C^{2}(M \backslash \Omega)$ with properties that mimic those of a good distance function at infinity. General functiontheoretic criteria in this spirit were developed by H. Donnelly [55] $(c=0)$, and K.D. Elworthy and F-Y. Wang [60] $(c>0)$. In particular, as a corollary of the main criterion in [55], a manifold with Ric $\geq 0$ and maximal volume growth (that is, $\operatorname{vol}\left(B_{r}\right) \geq D r^{m}$ for some $D>0)$ satisfies $\sigma(M)=[0,+\infty)$. It is important to observe that no pole assumption is required in Donnelly's paper and, as far as we know, it is the first result of this kind in the literature. Since then, major recent improvements have been made in a series of papers ([130], [137], [95], [32]), whose guiding idea is to replace the $L^{2}$-norm in (113) with the $L^{1}$-norm. The starting point is the following result of K.T. Sturm:

Theorem 6.2 ([130]). Let $M$ be a complete non-compact manifold with $\mathrm{Ric} \geq-K$ for some $K \geq 0$. If the volume of $M$ grows uniformly subexponentially ${ }^{7}$, then the $L^{p}$-spectra ${ }^{8}$ are the same for all $p \in[1,+\infty]$.

As first pointed out in [137], the key fact here is that in the computation of the $L^{1}$ spectrum one can effectively use smoothed distance functions to construct a sequence $\left\{u_{j}\right\}$ that satisfy $\left\|\Delta u_{j}+\lambda u_{j}\right\|_{1}=o\left(\left\|u_{j}\right\|_{1}\right)$ as $j \rightarrow+\infty$. In this way, in [137], [95] the authors proved the following striking

\footnotetext{
${ }^{6}$ Here, $\sigma(M)$ for incomplete $M$ is defined as the spectrum of the Friedrichs extension of $\left(-\Delta, C_{c}^{\infty}(M)\right)$.

${ }^{7}$ We say that the volume grows uniformly subexponentially if for each $\varepsilon>0$ there exists $C_{\varepsilon}>0$ such that

$$
\operatorname{vol}\left(B_{r}(x)\right) \leq C_{\varepsilon} e^{\varepsilon r} \operatorname{vol}\left(B_{1}(x)\right) \quad \forall x \in M
$$

${ }^{8}$ Denoting with $\Delta_{2}$ the Laplace operator on $L^{2}$, the semigroup $e^{t \Delta_{2}}$ extends to a strongly continuous contraction semigroup $T_{p}$ on $L^{p}(M)$ for all $p \in[1,+\infty)$. By definition, the $L^{p}$-spectrum is the spectrum of the generator $\Delta_{p}$ of $T_{p}$, and $\Delta_{\infty}$ is the adjoint of $\Delta_{1}$.
} 
Theorem 6.3 ([137, 95]). Let $M$ be complete and suppose that

$$
\liminf _{\rho(x) \rightarrow+\infty} \operatorname{Ric}_{x}=0
$$

in the sense of quadratic forms. Then, $\sigma(M)=[0,+\infty)$.

Besides on [55], this result improves on [90] and [62] (see also Corollary 2.17 in [26]), where $M$ was assumed to have a pole. Further refinements of (115) have been given in [32].

In some instances, the uniformity of the subexponential volume growth in Theorem 6.2 is quite difficult to check. This is the case of gradient Ricci solitons or of proper selfshrinkers of the mean curvature flow in $\mathbb{R}^{n}$, for which partial results in [95] suggest that $\sigma(M)=[0,+\infty)$. In [32], N. Charalambous and Z. Lu proved an important generalization of Weyl's criterion that allowed them to deal with these relevant examples:

Theorem 6.4. Let $M$ be a complete manifold, and fix $\lambda \in \mathbb{R}_{0}^{+}$. If there exists a sequence $\left\{u_{j}\right\} \subset \operatorname{dom}(-\Delta)$ such that

$$
\left\|u_{j}\right\|_{\infty} \cdot\left\|\Delta u_{j}+\lambda u_{j}\right\|_{1}=o\left(\left\|u_{j}\right\|_{2}^{2}\right) \quad \text { as } j \rightarrow+\infty
$$

then $\lambda \in \sigma(M)$.

We observe that $\operatorname{dom}(-\Delta)$ and $\sigma(M)$ are referred to the $L^{2}$-spectrum. This criterion is quite flexible: it allows, for example, to replace (115) by the weaker conditions

$$
\left\{\begin{array}{l}
\liminf _{\rho(x) \rightarrow+\infty} \operatorname{Ric}(\nabla \rho, \nabla \rho) \geq 0 \\
\text { either } \operatorname{vol}(M)=+\infty
\end{array} \quad \text { or } \quad \lim _{r \rightarrow+\infty} \frac{-\log \left(\operatorname{vol}(M) \backslash \operatorname{vol}\left(B_{r}\right)\right)}{r}=0\right.
$$

where $\rho(x)$ is the distance from some origin $o$ and $x \notin \operatorname{Cut}(o)$. As another relevant application, we quote the following beautiful result by L. Silvares [128], that improves on $[32,95]$. We recall that a weighted manifold $(M, g, f)$ is a Riemannian manifold $(M, g)$ endowed with a reference function $f$, and that its Bakry-Emery Ricci tensor is defined as

$$
\operatorname{Ric}_{f}=\operatorname{Ric}+\operatorname{Hess} f .
$$

Theorem 6.5. [128] Let $(M, g, f)$ be a complete weighted manifold. If $\mathrm{Ric}_{f} \geq \frac{1}{2}$ and $|\nabla f|^{2} \leq f$, then $\sigma(M)=[0,+\infty)$.

Remark 6.6. For weighted manifolds, similar question can be asked for the spectrum of the drifted Laplacian $\Delta_{f}=\Delta-\langle\nabla f, \nabla \cdot\rangle$, and the situation is quite different from that for $\Delta$. Interesting results can be found in $[128,43]$.

A typical case when the assumptions in Theorem 6.5 are met is that of shrinking Ricci solitons, for which up to rescaling $g$ and translating $f$, Ric $f=\frac{1}{2}$ and $|\nabla f|^{2} \leq f$. Therefore, the spectrum of a complete, shrinking Ricci soliton is $[0,+\infty$ ), as shown in [32] (and, under a further growth condition on $f$, in [95]).

Another class of manifolds that share a great similarity with shrinking Ricci solitons is the class of self-shrinkers for the mean curvature flow. We recall that $X: M^{m} \rightarrow \mathbb{R}^{n}$ is called a self-shrinker if its mean curvature vector satisfies

$$
H=-\frac{X^{\perp}}{2}
$$

$X^{\perp}$ being the orthogonal component of the position vector. If $X$ is proper, the function $f=|X|^{2} / 4$ is a proper exhaustion satisfying $|\nabla f|^{2} \leq f$. Although it is not true that $\operatorname{Ric}_{f} \geq \frac{1}{2}$, nevertheless one can still prove the following result:

Theorem 6.7. [127] If $M \rightarrow \mathbb{R}^{n}$ is a properly immersed self-shrinker for the mean curvature flow, then $\sigma(M)=[0,+\infty)$. 
In all of the examples, the fact that $\sigma(M)=[0,+\infty)$ depends on the existence of an exhaustion $\gamma$ such that $|\Delta \gamma|$ and $|\nabla \gamma|^{2}-1$ are suitably small in an integral sense, coupled with a subexponential growth of $V(r)=\operatorname{vol}(\{\gamma \leq r\})$ (if $V(\infty)=\infty$ ) or otherwise a subexponential decay of $V(\infty)-V(r)$, in the spirit of Donnelly and Elworthy-Wang's criteria (and its $L^{1}$-versions in [32]). In the lack of subexponential volume growth, without the pole assumption very few is known, and is still the subject of an active area of research. It has been conjectured in [32] that if $M$ is complete with $\operatorname{vol}(M)=+\infty$, condition Ric $\geq-K$ for some $K>0$ imply that the essential spectrum is $[c,+\infty)$, for some $c \geq 0$. Note that the requirement $\operatorname{vol}(M)=+\infty$ is needed in view of the examples constructed by J. Lott in $[94]^{9}$. However, very recently, in [124] the authors showed that the above conjecture is false. More precisely, they proved the following beautiful:

Theorem 6.8. Let $\left(M, g_{0}\right)$ be a complete, noncompact Riemannian manifold, and let $n \in \mathbb{N}$. If $g_{0}$ has bounded curvature and positive injectivity radius, there is a metric $g$ on $M$ such that $(M, g)$ has bounded curvature, positive injectivity radius and, for the Laplacian of the metric $g, \sigma_{\mathrm{ess}}(M)$ has at least $n$ gaps.

In particular, Riemannian coverings $\left(M, g_{0}\right)$ of compact manifolds satisfy the assumptions of the theorem. In view of this result, one can ask whether the gaps stay below some fixed threshold. We are tempted to suggest the following

Conjecture 6.9. Let $M$ be a complete manifold with Ric $\geq-(m-1) \kappa$ for some $\kappa>0$. If $\operatorname{vol}(M)=+\infty$, then

$$
\sigma_{\mathrm{ess}}(M) \supset\left[\frac{(m-1)^{2} \kappa}{4},+\infty\right) .
$$

6.1. Immersed submanifolds, I: the ball property. We now move to investigate more closely the spectrum of isometrically immersed submanifolds, in particular minimal ones. As in the case of shrinking Ricci solitons and self-shrinkers, it may happen that $\sigma(M)=[0,+\infty)$ even without a good control on the curvature. Another example, which we think to be quite curious, is that of graphs, for which we have the following recent result:

Theorem 6.10 (Matos-Montenegro [103]). The spectrum of any complete smooth graph over a domain $\Omega \subset \mathbb{R}^{m}$ is $[0,+\infty)$.

Note that no assumption is required on the graph function. In particular, if $\Omega \neq \mathbb{R}^{m}$, the graph may oscillate fastly when approaching $\partial \Omega$. Another examples for which $\sigma(M)=$ $[0,+\infty)$ but no reasonable control on the curvature is available are described in (7.1), and are a consequence of a simple but flexible criterion, called the ball property, which we now describe. We begin with the following

Definition 6.11. A Riemannian manifold $M$ has the ball property if there exists $R>0$ and a collection of disjoint balls $\left\{B_{R}^{M}\left(x_{j}\right)\right\}_{j=1}^{+\infty}$ of radius $R$ centered at $x_{j}$ such that for some constants $C>0, \delta \in(0,1)$, possibly depending on $R$,

$$
\operatorname{Vol}\left(B_{\delta R}^{M}\left(x_{j}\right)\right) \geq C^{-1} \operatorname{Vol}\left(B_{R}^{M}\left(x_{j}\right)\right) \quad \forall j \in \mathbb{N} .
$$

Observe that (116) is not a doubling condition since it needs to hold only along the sequence $\left\{x_{j}\right\}$ and the constant $C$ may depend on $R$. The importance of the ball property is that its validity implies that the essential spectrum is nonempty.

\footnotetext{
${ }^{9}$ The volume condition was absent in the original formulation of the conjecture in [32], but was pointed out to us by the authors themselves after they discovered J. Lott's paper.
} 
Theorem 6.12 (Bessa-Jorge-Mari-[17]). If a Riemannian manifold has the ball property (with parameters $R, \delta, C$ ), then

$$
\inf \sigma_{\text {ess }}(-\Delta) \leq \frac{C}{R^{2}(1-\delta)^{2}}
$$

The well-known Bishop-Gromov volume comparison theorem, see [27], [75], [115], shows that any complete non-compact Riemannian $m$-manifold $M$ with Ricci curvature bounded from below has the ball property, therefore it has non-empty essential spectrum. This was known to H. Donnelly, that proved sharp results in the class of manifolds with Ricci curvature bounded below. Donnelly showed that the essential spectrum of a complete non-compact Riemannian $m$-manifold $M$ with Ricci curvature $\operatorname{Ric}_{M} \geq-(m-1) c^{2}>-\infty$ intersects the interval $\left[0,(m-1)^{2} c^{2} / 4\right]$, [52, Thm. 3.1]. However, there exist examples of complete non-compact Riemannian manifolds with the ball property and inf Ric $=-\infty$. For instance, the examples of Jorge-Xavier of minimal surfaces between two parallel planes that have Ricci curvature satisfying inf Ric $=-\infty$, see [20], [120] and some of them have the ball property and therefore have non-empty essential spectrum. H. Rosenberg and E. Toubiana, in [121], constructed a complete minimal annulus between two parallel planes of $\mathbb{R}^{3}$ such that the immersion is proper in the slab. The Jorge, Xavier's and Rosenberg, Toubiana's examples are constructed with a flexible method depending on a chosen set of parameters and we will show that, depending on this choice of parameters, the spectrum of the complete minimal surfaces immersed in the slab can be the half-line $[0, \infty)$.

Other examples of manifolds with the ball property are, for instance, the non-proper submanifolds with locally bounded geometry. An isometric immersion $\varphi: M \rightarrow N$ is said to have locally bounded geometry if for each compact set $W \subset N$ there is a constant $\Lambda=\Lambda(W)$ such that

$$
\left\|\alpha_{\varphi}\right\|_{L^{\infty}\left(\varphi^{-1}(W)\right)} \leq \Lambda .
$$

Here $\alpha_{\varphi}$ is the second fundamental form of the immersion $\varphi$.

To complete this section about the ball property we will prove the following result about the spectrum of non-proper submanifolds with locally bounded geometry.

Theorem 6.13 (Bessa-Jorge-Mari-[17]). Let $\varphi: M \rightarrow N$ be an isometric immersion with locally bounded geometry of an open Riemannian m-manifold $M$ into a complete Riemannian n-manifold $N$. If the immersion is non-proper, then $M$ has the ball property. Thus, it has non empty essential spectrum.

6.2. Proof of Theorem 6.12. In this section we show that the ball property, introduced in Definition 6.11, implies the existence of elements in the essential spectrum of $-\Delta$. Let $M$ be a Riemannian manifold with the ball property, this is, there exists $R>0$ and a collection of disjoint balls $\left\{B_{R}^{M}\left(x_{j}\right)\right\}_{j=1}^{\infty}$ such that for some constants $C>0$ and $\delta \in(0,1)$ the inequalities

$$
\operatorname{vol}\left(B_{\delta R}^{M}\left(x_{j}\right)\right) \geq C^{-1} \operatorname{vol}\left(B_{R}^{M}\left(x_{j}\right)\right), \quad j=1,2, \ldots
$$

hold. For each $j$, define the compactly supported, Lipschitz function $\phi_{j}(x)=\zeta\left(\rho_{j}(x)\right)$, where $\rho_{j}(x)=\operatorname{dist}\left(x, x_{j}\right)$ and

$$
\zeta(t)=\left\{\begin{array}{cll}
1, & \text { if } \quad t \leq \delta R . \\
\frac{R-t}{R(1-\delta)}, & \text { if } \quad t \in[\delta R, R] . \\
0, & \text { if } \quad t \geq R .
\end{array}\right.
$$


Observe that $\left|\zeta^{\prime}\right| \leq \frac{1}{R(1-\delta)}$. By the ball property (116),

$$
\begin{aligned}
I_{\lambda}\left(\phi_{j}, \phi_{j}\right) & =\int_{B_{R}^{M}\left(x_{j}\right)}\left|\nabla \phi_{j}\right|^{2}-\lambda \int_{B_{R}^{M}\left(x_{j}\right)} \phi_{j}^{2} \\
& \leq \frac{\operatorname{Vol}\left(B_{R}^{M}\left(x_{j}\right)\right)}{R^{2}(1-\delta)^{2}}-\lambda \operatorname{vol}\left(B_{\delta R}^{M}\left(x_{j}\right)\right) \\
& \leq \operatorname{Vol}\left(B_{R}^{M}\left(x_{j}\right)\right)\left(\frac{1}{R^{2}(1-\delta)^{2}}-\lambda C^{-1}\right) \\
& <0
\end{aligned}
$$

provided that $\lambda>C /\left(R^{2}(1-\delta)^{2}\right)$.

Since $\left\{\phi_{j}\right\}$ span an infinite-dimensional subspace of the domain of $-\Delta$, the Friedrichs extension of the operator $-(\Delta+\lambda)$ has infinite index, or equivalently, $-\Delta$ has infinite eigenvalues below $\lambda$, for each $\lambda>C /(R(1-\delta))^{2}$. By the Min-Max Theorem, see [52, Prop. $2.1 \& 2.2]$, [116, Section 3], the inequality inf $\sigma_{\text {ess }}(-\Delta) \leq C /(R(1-\delta))^{2}$ follows.

Remark 6.14. In virtue of the well known Bishop-Gromov volume comparison theorem, [27], [75], [115], all Riemannian n-manifolds $M$ with Ricci curvature bounded below Ric $_{M} \geq-(n-1) k^{2}$ has the ball property. In fact, if we denote by $\operatorname{Vol}_{\kappa}(r)$ the volume of a geodesic ball of radius $r$ in the hyperbolic space $\mathbb{H}_{\kappa}^{n}$ of constant sectional curvature $\kappa$. By the Bishop-Gromov volume comparison theorem, the ratio $\operatorname{Vol}\left(B_{r}\left(x_{j}\right)\right) / \operatorname{Vol}_{\kappa}(r)$ is non-increasing on $[0, R]$. Hence, for each $\delta>0$

$$
\operatorname{Vol}\left(B_{\delta R}^{M}\left(x_{j}\right)\right) \geq \frac{\operatorname{Vol}_{\kappa}(\delta R)}{\operatorname{Vol}_{\kappa}(R)} \operatorname{Vol}\left(B_{R}^{M}\left(x_{j}\right)\right)=C(\delta, R)^{-1} \operatorname{Vol}\left(B_{R}^{M}\left(x_{j}\right)\right) .
$$

\section{Jorge-Xavier \& Rosenberg-Toubiana minimal surfaces}

We will need to give a brief description of the complete minimal surfaces between two parallel planes, constructed by Jorge-Xavier in [84]. They constructed complete minimal immersions of the disk $\varphi: \mathbb{D} \rightarrow \mathbb{R}^{3}$ with $\mathbb{R}^{3}, \varphi(M) \subset\left\{(x, y, z) \in \mathbb{R}^{3}:|z|<1\right\}$. Let $\left\{D_{n} \subset \mathbb{D}\right\}$ be a sequence of closed disks centered at the origin such that $D_{n} \subset \operatorname{int}\left(D_{n+1}\right)$, $\cup D_{n}=\mathbb{D}$. Let $K_{n} \subset D_{n}$ be a compact set so that $K_{n} \cap D_{n-1}=\emptyset$ and $D_{n} \backslash K_{n}$ is connected as in the figure 1 . below.

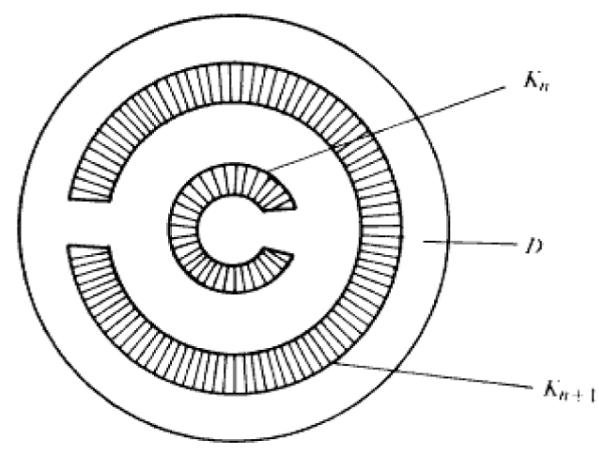

Fig. 1. The compact sets $K_{n}$.

By Runge's Theorem, [80, p. 96], there exists a holomorphic function $h: \mathbb{D} \rightarrow \mathbb{C}$ such that $\left|h-c_{n}\right|<1$ on $K_{n}$, for each $n$. Letting $g=e^{h}$ and $f=e^{-h}$ and setting

$$
\phi=\left(f\left(1-g^{2}\right) / 2, i \cdot f\left(1+g^{2}\right) / 2, f g\right),
$$

by the Weierstrass representation, one has that $\varphi=\operatorname{Re} \int \phi: \mathbb{D} \rightarrow \mathbb{R}^{3}$ is a minimal surface with bounded third coordinate. Let $r_{n}$ denote the Euclidean distance between the inner 
and the outer circle of $K_{n}$ and for each $n$ choose a constant $c_{n}$ such that

$$
\sum_{n \text { even }}^{+\infty} r_{n} e^{c_{n}-1}=+\infty, \quad \sum_{n \text { odd }}^{+\infty} r_{n} e^{c_{n}-1}=+\infty .
$$

Condition (120) implies that this minimal surface is complete.

The induced metric $\mathrm{d} s^{2}$ by this minimal immersion is conformal to the Euclidean metric $|\mathrm{d} z|^{2}$ given by $\mathrm{d} s^{2}=\lambda^{2}|\mathrm{~d} z|^{2}$, where

$$
\lambda(z)=\frac{1}{2}\left(\left|e^{h(z)}\right|+\left|e^{-h(z)}\right|\right) .
$$

The choice of the compact subsets $K_{n} \subset D_{n}$ with width $r_{n}$ and the set of constants $c_{n}$ satisfying (120) and yielding a complete minimal surface of $\mathbb{R}^{3}$ between two parallel planes is what we are calling a choice of parameters, $\left.\left(\left\{r_{n}, c_{n}\right)\right\}\right)$, in Jorge-Xavier's construction. We should give a brief description of Rosenberg-Toubiana construction of a complete minimal annulus properly immersed into a slab of $\mathbb{R}^{3}$, see details in [121]. They start considering a labyrinth in the annulus

$$
\mathbb{A}(1 / c, c)=\{z \in \mathbb{C}: 1 / c<|z|<c\}, c>1
$$

composed by compact sets $K_{n}$ contained in the annulus $\mathbb{A}(1, c)$ and compact sets $L_{n}=$ $\left\{1 / \bar{z}:-z \in K_{n}\right\}$ contained in the annulus $\mathbb{A}(1 / c, 1)$ as in the figure 2 . below. The compact sets $L_{n}$ are converging to the boundary $|z|=1 / c$ and the compact sets $K_{n}$ are converging to the boundary $|z|=c$.

They needed two non-vanishing holomorphic functions $f, g: \mathbb{A}(1 / c, c) \rightarrow \mathbb{C}$, in order to construct a minimal surface via Weierstrass representation formula, so that the resulting minimal surface is geodesically complete and properly immersed into a slab. They construct $f$ and $g$ satisfying $f(z) \cdot g(z)=1 / z$ where $\left|g(z)-e^{2 c_{n}}\right|<1$ on $K_{n}$ and $\left|g(z)-e^{-2 c_{n}}\right|<1$ on $L_{n}$, where $\left\{c_{n}\right\}$ is a sequence of positive numbers such that

$$
\sum_{n}^{\infty} r_{n} e^{2 c_{n}}=\infty, \quad \sum_{n}^{\infty} s_{n} e^{2 c_{n}}=\infty
$$

and $r_{n}$ and $s_{n}$ are the width of $K_{n}$ and $L_{n}$ respectively. The induced metric by the immersion on the annulus $\mathbb{A}(1 / c, c)$ is $d s^{2}=\lambda^{2}|d z|^{2}$ where

$$
\lambda=\frac{1}{2|z|}\left(\frac{1}{|g(z)|}+|g(z)|\right) \text {. }
$$

On $K_{n}$ we have

$$
e^{2 c_{n}} \geq\left(1+\frac{e^{2 c_{n}}}{2}\right) \geq \lambda \geq \frac{1}{2|c|}\left(e^{2 c_{n}}-1\right)
$$

The choice of parameters $\left\{\left(r_{n}, c_{n}\right)\right\}$ in Jorge-Xavier's construction or $\left\{\left(r_{n}, s_{n}, c_{n}\right)\right\}$ in Rosenberg-Toubiana's construction gives information about the essential spectrum of the resulting surfaces. In the next result, set $\lambda_{n}:=\sup _{z \in K_{n}} \lambda(z)$.

Theorem 7.1 (Bessa-Jorge-Mari-[17]). Let $\varphi: \Omega \rightarrow \mathbb{R}^{3}$ be either Jorge-Xavier's or RosenbergToubiana's ${ }^{10}$ complete minimal surface immersed into the slab with defining parameters $\left\{\left(r_{n}, c_{n}\right)\right\}$ or $\left\{\left(r_{n}, s_{n}, c_{n}\right)\right\}$. If $\lim \sup \lambda_{n} r_{n}=\infty$, then $\sigma_{\mathrm{ess}}(-\Delta)=[0, \infty)$. And if $\limsup \lambda_{n} r_{n}>0$, then $\varphi(\mathbb{D})$ or $\varphi(\mathbb{A}(1 / c, c))$ has the ball property, therefore $\sigma_{\mathrm{ess}}(-\Delta) \neq \emptyset$.

At points $z \in K_{n}$ we have $e^{1+c_{n}} \geq \lambda(z) \geq \frac{1}{2} e^{c_{n}-1}$, therefore

$$
e^{c_{n}+1} \geq \lambda_{n} \geq e^{c_{n}} / 2 e \text {. }
$$

${ }^{10}$ Whether $\Omega=\mathbb{D}$ or $\left.\Omega=\mathbb{A}(1 / c, c)\right)$. 
If $c_{n}=-\log \left(r_{n}^{2}\right)$ we have that the parameters $\left\{\left(r_{n}, c_{n}\right)\right\}$ satisfies (120) and $\lambda_{n} r_{n}=$ $1 /\left(2 e r_{n}\right)$. Thus $\lim \sup \lambda_{n} r_{n}=\infty$ yielding a complete minimal surface between two parallel planes with spectrum $\sigma(-\Delta)=[0, \infty)$. In the original construction in [84], Jorge-Xavier choose $c_{n}=-\log r_{n}$ that yields $e \geq r_{n} \lambda_{n} \geq 1 / 2 e$ and the resulting minimal surfaces has nonempty essential spectrum.

7.1. Application of the ball property to minimal surfaces in the slab. In this section we will show that, for a suitable choice of their defining parameters, the JorgeXavier and Rosenberg-Toubiana complete minimal surfaces immersed into slabs of $\mathbb{R}^{3}$ have the ball property. Denoting by $\varphi: \mathbb{D} \rightarrow\left\{\left(x_{1}, x_{2}, x_{3}\right):\left|x_{3}\right|<1\right\}$ and $\varphi: \mathbb{A}(1 / c / c) \rightarrow$ $\left\{\left(x_{1}, x_{2}, x_{3}: 1 / c<x_{3}<c\right\}\right.$ with parameters $\left\{\left(r_{n}, c_{n}\right)\right\},\left\{\left(r_{n}, s_{n}, c_{n}\right)\right\}$ respectively, the examples of Jorge-Xavier and Rosenberg-Toubiana, we shall show that with the choice $c_{n}=-\log \left(r_{n}^{2}\right)$, we have that $0=\inf \sigma_{\text {ess }}(-\Delta)$ in both surfaces. The induced metric $\mathrm{d} s^{2}$ in Jorge-Xavier minimal immersion is conformal to the Euclidean metric $|\mathrm{d} z|^{2}$. More precisely, $\mathrm{d} s^{2}=\lambda^{2}|\mathrm{~d} z|^{2}$, where

$$
\lambda=\frac{1}{2}\left(\left|e^{h}\right|+\left|e^{-h}\right|\right) .
$$

At points of $K_{n}$,

$$
e^{1+c_{n}} \geq \lambda \geq \frac{1}{2} e^{c_{n}-1}
$$

thus,

$$
e^{2+2 c_{n}}|\mathrm{~d} z|^{2} \geq \mathrm{d} s^{2}=\lambda^{2}|\mathrm{~d} z|^{2} \geq \frac{1}{4} e^{2 c_{n}-2}|\mathrm{~d} z|^{2} .
$$

Choosing $c_{n}=-\log \left(r_{n}^{2}\right)$ and letting $I_{n}$ be the segment of the real axis that crosses $K_{n}$ one has that the length $\ell\left(I_{n}\right)$ of this segment in the metric $\mathrm{d} s^{2}$ has the following lower and upper bound

$$
\frac{e^{2}}{r_{n}^{4}} \geq \ell\left(I_{n}\right) \geq r_{n} e^{c_{n}-1} \geq \frac{e^{-1}}{r_{n}} .
$$

Let $p_{n}$ be the center of the $I_{n}$ and denote by $B_{R}^{\mathrm{d} s^{2}}\left(p_{n}\right)$ and $B_{R}^{|\mathrm{d} z|^{2}}\left(p_{n}\right)$ the geodesic balls of radius $R$ and center $p_{n}$ with respect to the metric $\mathrm{d} s^{2}$ and the metric $|\mathrm{d} z|^{2}$ respectively. Giving $R>0$, there exists $n_{R}$ such that for all $n \geq n_{R}$ the geodesic ball $B_{R}^{\mathrm{d} s^{2}}\left(p_{n}\right) \subset K_{n}$ for all $n \geq n_{R}$. Indeed, since $r_{n} \rightarrow 0$ as $n \rightarrow \infty$, just choose $n_{R}$ be such that $r_{n_{R}} \leq \frac{e^{-1}}{3 R}$. Moreover, these inclusions

$$
B_{R /\left(e^{\left.1+c_{n}\right)}\right.}^{\mid \mathrm{d} z 2^{2}}\left(p_{n}\right) \subset B_{R}^{\mathrm{d} s^{2}}\left(p_{n}\right) \subset B_{2 R /\left(e^{c_{n}-1}\right)}^{\mid \mathrm{d} z 2^{2}}\left(p_{n}\right)
$$

holds. Therefore, for $\delta \in(0,1)$, we have

$$
\begin{aligned}
\operatorname{vol}_{\mathrm{d} s^{2}}\left(B_{\delta R}^{\mathrm{d} s^{2}}\left(p_{n}\right)\right) & \geq \operatorname{vol}_{\mathrm{d} s^{2}}\left(B_{\delta R /\left(e^{1+c_{n}}\right)}^{\mid \mathrm{d} z 2^{2}}\left(p_{n}\right)\right) \\
& \geq \frac{1}{4} e^{2 c_{n}-2} \operatorname{vol}_{|\mathrm{d} z|^{2}}\left(B_{\delta R /\left(e^{\left.1+c_{n}\right)}\right.}^{|\mathrm{d} z|^{2}}\left(p_{n}\right)\right) \\
& =\frac{1}{4 e^{4}} \operatorname{vol}_{|\mathrm{d} z|^{2}}\left(B_{\delta R}^{|\mathrm{d} z|^{2}}\left(p_{n}\right)\right),
\end{aligned}
$$

and

$$
\begin{aligned}
\operatorname{vol}_{\mathrm{d} s^{2}}\left(B_{R}^{\mathrm{d} s^{2}}\left(p_{n}\right)\right) & \leq \operatorname{vol}_{\mathrm{d} s^{2}}\left(B_{2 R /\left(e^{c_{n}-1}\right)}^{|\mathrm{d} z|^{2}}\left(p_{n}\right)\right) \\
& \leq e^{2 c_{n}+2} \operatorname{vol}_{|\mathrm{d} z|^{2}}\left(B_{2 R /\left(e^{c_{n}-1}\right)}^{|\mathrm{d} z|^{2}}\left(p_{n}\right)\right) \\
& =4 e^{4} \operatorname{vol}_{|\mathrm{d} z|^{2}}\left(B_{R}^{|\mathrm{d} z|^{2}}\left(p_{n}\right)\right)
\end{aligned}
$$


From (123) and (124) we have

$$
\operatorname{vol}_{\mathrm{d} s^{2}}\left(B_{\delta R}^{\mathrm{d} s^{2}}\left(p_{n}\right)\right) \geq \frac{\delta^{2}}{e^{10}} \cdot \operatorname{vol}_{\mathrm{d} s^{2}}\left(B_{R}^{\mathrm{d} s^{2}}\left(p_{n}\right)\right) .
$$

This shows that Jorge-Xavier minimal surfaces with those choices of $c_{n}$ above has the ball property, (along the sequence $p_{n}$, for $n \geq n_{R}$ ), with parameters $R, \delta$ and $C=e^{10} / \delta^{2}$. By Theorem 6.12,

$$
\inf \sigma_{e s s}(-\Delta) \leq \frac{C}{R^{2}(1-\delta)^{2}} .
$$

Letting $R \rightarrow \infty$, we conclude that $0 \in \sigma_{\text {ess }}(-\Delta)$.

Likewise, the induced metric in the Rosenberg-Toubiana's complete minimal annulus properly immersed into a slab of $\mathbb{R}^{3}$ is $\lambda^{2}|\mathrm{~d} z|^{2}$, where $\lambda=\frac{1}{2|z|}\left(\frac{1}{|g(z)|}+|g(z)|\right)$. On $K_{n}$ we have

$$
e^{2 c_{n}} \geq\left(1+\frac{e^{2 c_{n}}}{2}\right) \geq \lambda \geq \frac{1}{2|c|}\left(e^{2 c_{n}}-1\right)
$$

Letting $I_{n}$ be the segment of the real axis crossing $K_{n}$ and $p_{n}$ the middle point of $I_{n}$ we have that the geodesic ball (in the metric $\mathrm{d} s^{2}$ ) with radius $R>0$ and center $p_{n}$ is contained in $K_{n}$, for sufficiently large $n$,

$$
B_{R}^{\mathrm{d} s^{2}}\left(p_{n}\right) \subset K_{n} .
$$

Moreover,

$$
B_{\frac{1}{e^{2 c_{n}}} R}^{|\mathrm{d} z|^{2}}\left(p_{n}\right) \subset B_{R}^{\mathrm{d} s^{2}}\left(p_{n}\right) \subset B_{\frac{2|c|}{e^{2 c_{n}}-1} R}^{|\mathrm{d} z|^{2}}\left(p_{n}\right)
$$

Thus

$$
\operatorname{vol}_{\mathrm{d} s^{2}}\left(B_{\delta R}^{\mathrm{d} s^{2}}\left(p_{n}\right)\right) \geq \operatorname{vol}_{\mathrm{d} s^{2}}\left(B_{\frac{1}{e^{2 c_{n}}} \delta R}^{|\mathrm{d} z|^{2}}\left(p_{n}\right)\right) \geq \frac{\left(e^{2 c_{n}}-1\right)^{2}}{4|c|^{2} e^{4 c_{n}}} \operatorname{vol}_{|\mathrm{d} z|^{2}}\left(B_{\delta R}^{|\mathrm{d} z|^{2}}\left(p_{n}\right)\right)
$$

and

$$
\operatorname{vol}_{\mathrm{d} s^{2}}\left(B_{R}^{\mathrm{d} s^{2}}\left(p_{n}\right)\right) \leq \operatorname{vol}_{\mathrm{d} s^{2}}\left(B_{\frac{2|c|}{e^{2 c_{n}-1}} R}^{|\mathrm{d}|^{2}}\left(p_{n}\right)\right) \leq \frac{4|c|^{2} e^{4 c_{n}}}{\left(e^{2 c_{n}}-1\right)^{2}} \operatorname{vol}_{|\mathrm{d} z|^{2}}\left(B_{R}^{|\mathrm{d} z|^{2}}\left(p_{n}\right)\right) .
$$

Therefore, for $n$ so that $1-r_{n} \geq 2 / 3$ we have

$$
\operatorname{vol}_{\mathrm{d} s^{2}}\left(B_{\delta R}^{\mathrm{d} s^{2}}\left(p_{n}\right)\right) \geq \frac{\delta^{2}}{81|c|^{4}} \operatorname{vol}_{\mathrm{d} s^{2}}\left(B_{R}^{\mathrm{d} s^{2}}\left(p_{n}\right)\right) .
$$

This shows that Rosenberg-Toubiana minimal surfaces with those choices of $c_{n}$ have the ball property, (along the sequence $p_{n}$ ), with parameters $R, \delta$ and $C=81|c|^{4} / \delta^{2}$. By Theorem 6.12 ,

$$
\inf \sigma_{e s s}(-\Delta) \leq \frac{C}{R^{2}(1-\delta)^{2}}
$$

Again, letting $R \rightarrow \infty$, we conclude that $0 \in \sigma_{e s s}(-\Delta)$. This finishes the proof.

We conclude this section calling the attention to an example of a bounded minimal surface $\varphi: M \rightarrow \mathbb{R}^{3}$ with $\operatorname{dim}_{\mathcal{H}}(\varphi(M))=3$, which is not a covering and $\sigma_{\text {ess }}(-\Delta) \neq \emptyset$. P. Andrade [7], constructed a complete minimal immersion $\varphi: \mathbb{C} \rightarrow \mathbb{R}^{3}$ with bounded curvature with the property that $\overline{\varphi(\mathbb{C})}$ was an unbounded subset of the Euclidean space $\mathbb{R}^{3}$ with $\operatorname{vol}_{3}(\overline{\varphi(\mathbb{C})})=\infty$. In other words, he constructed a dense complete minimal surface with bounded curvature thus, with the ball property. However, the restriction of the parametrization of Andrade's surface to a strip $U=\{z \in \mathbb{C}:|\operatorname{Re} z|<1\}$, yields a bounded, simply-connected minimal immersion with the ball property and dense in a bounded subset of $\mathbb{R}^{3}$. To give more details, we will keep Andrade's notation, thus, here and only here, $H$ will be a holomorphic function. 
Example 7.2. Choose $r_{1}, r_{2}>0$ such that $r_{1} / r_{2}$ is irrational and $r_{1} / r_{2}<1$, and set $\mathrm{d}=r_{2}-r_{1}$. Define the map $\chi: \mathbb{C} \rightarrow \mathbb{R}^{3}=\mathbb{C} \times \mathbb{R}, \chi(z)=(L(z)-\overline{H(z)}, h(z))$, for the following choice of holomorphic functions $L, H$ and harmonic function $h$,

$$
\begin{gathered}
L(z)=\left(r_{1}-r_{2}\right) e^{z}, \quad H(z)=-\mathrm{d} e^{\left(\frac{r_{1}}{r_{2}}-1\right) z}, \text { and } \\
h(z)=4\left(\frac{\mathrm{d}}{r_{2}}\right)^{1 / 2}\left|\frac{r_{2}}{r_{1}}\right|\left|r_{2}-r_{1}\right| \Re\left(i e^{\frac{r_{1}}{2 r_{2}} z}\right),
\end{gathered}
$$

where $\Re$ means the real part. Then, a straightforward computation gives that

$$
\left|L^{\prime}(z)\right|+\left|H^{\prime}(z)\right|>0, \quad L^{\prime} H^{\prime}=\left(\frac{\partial h}{\partial z}\right)^{2} \quad \text { on } \mathbb{C},
$$

the necessary and sufficient conditions on $\chi$ to be a conformal minimal immersion of $\mathbb{C}$ in $\mathbb{R}^{3}$. Restricting $\chi$ to the region $U=\{u+i v:|u|<1\}$, we get a bounded, simply connected minimal immersion $\varphi=\chi_{\mid U}$. For each fixed $u \in(-1,1), \varphi(u+i v)$ is a dense immersed trochoid in the cylinder $\Gamma_{u}=\left[B_{s_{1}(u)} \backslash B_{s_{2}(u)}\right] \times(-l(u), l(u))$, where $s_{1}, s_{2}, l$ are explicit functions of $u$ depending on $r_{1}$ and $r_{2}$. Therefore, $\lim \varphi$ is dense in the open subset $\bigcup_{u \in(-1,1)} \Gamma_{u}$ of $\mathbb{R}^{3}$, which gives $\operatorname{dim}_{\mathcal{H}}(\lim \varphi)=3$. Moreover, the induced metric $\mathrm{d} s^{2}$ satisfies

$$
\begin{aligned}
\mathrm{d} s^{2} & =\left(\left|L^{\prime}\right|+\left|H^{\prime}\right|\right)^{2}|\mathrm{~d} z|^{2} \\
& =\left(\left|r_{2}-r_{1}\right| e^{u}+d e^{\left(\frac{r_{1}}{r_{2}}-1\right) u}\right)^{2}|\mathrm{~d} z|^{2} \\
& \geq 4\left(r_{2}-r_{1}\right)^{2}|\mathrm{~d} z|^{2} .
\end{aligned}
$$

Considering $z_{k}=2 i k \in U$, each of the unit balls $B_{1}^{|\mathrm{d} z|^{2}}\left(z_{k}\right) \subseteq U$ in the metric $|\mathrm{d} z|^{2}$ contains a ball $B_{R}\left(z_{k}\right)$ in the metric $\mathrm{d} s^{2}$ of radius at least $R=2\left|r_{2}-r_{1}\right|$. Since the sectional curvature of $\chi$ satisfies

$$
K=-c_{1}\left(e^{\left(1-\frac{r_{1}}{4 r_{2}}\right) u}+c_{2} e^{\left(\frac{3 r_{1}}{4 r_{2}}-1\right) u}\right)^{-4}
$$

for some positive constants $c_{1}, c_{2}$, and $1-\frac{r_{1}}{4 r_{2}}$ and $\frac{3 r_{1}}{4 r_{2}}-1$ have opposite signs, then $\chi$ has globally bounded curvature. In particular, $\left\{B_{R}\left(z_{k}\right)\right\}$ is a collection of disjoint balls in $\left(U, \mathrm{~d} s^{2}\right)$ with uniformly bounded sectional curvature, therefore, $\sigma_{\mathrm{ess}}(-\Delta) \neq \emptyset$ on $\left(U, \mathrm{~d} s^{2}\right)$, by Theorem 6.12 and Remark 6.14.

7.2. Proof of Theorem 6.13. Consider a non-proper isometric immersion $\varphi: M \rightarrow$ $N$ with locally bounded geometry of a complete Riemannian manifold into a complete Riemannian manifold $N$. We are going to show that there exists a sequence $\left\{x_{j}\right\} \subset M$ a radius $R$, a constant $C>0$ and $\delta \in(0,1)$ such that

$$
\operatorname{vol}_{M}\left(B_{\delta R}^{M}\left(x_{j}\right)\right) \geq C^{-1} \operatorname{vol}_{M}\left(B_{R}^{M}\left(x_{j}\right)\right) .
$$

In other words, $M$ has the ball property. Let $y_{0} \in \lim \varphi$ and let $W \subset N$ be a compact subset with $y_{0} \in \operatorname{int}(W)$. Let $\Lambda_{0}=\Lambda_{0}(W)$ be such that $\left\|\alpha_{\varphi}\right\|_{L^{\infty}\left(\varphi^{-1}(W)\right)} \leq \Lambda_{0}$. The Gauss equation and the upper bound $\sup _{W}\left|K_{N}\right|<\infty$ of the sectional curvatures of $N$ on $W$ gives a positive number $b_{0}>0$ such that

$$
\sup _{x \in \varphi^{-1}(W)}\left|K_{M}(x)\right| \leq 2 \Lambda_{0}^{2}+\sup _{W}\left|K_{N}\right| \leq b_{0},
$$

where $K_{M}$ are the sectional curvatures of $M$. Therefore, each connected component $U \subset$ $\varphi^{-1}(W)$ has sectional curvatures uniformly bounded $\left|K_{U}\right| \leq b_{0}$. Set

$$
2 r_{0}=\min \left\{i_{W},\left(2 \Lambda_{0}\right)^{-1}, b_{0}^{-1 / 2} \cdot \cot ^{-1}\left(1 /\left(2 \sqrt{b_{0}}\right)\right), \operatorname{dist}_{N}\left(y_{0}, N \backslash W\right)\right\},
$$


where $i_{W}=\inf \left\{\operatorname{inj}_{N}(x), x \in W\right\}$. Let $B_{0}=\overline{B_{r_{0}}^{N}}\left(y_{0}\right)$ be the closure of the geodesic ball of $N$ with radius $r_{0}$ and center $y_{0}$. There exists a sequence of points $q_{j} \in M, q_{j} \rightarrow \infty$ in $M$ such that $\varphi\left(q_{j}\right) \rightarrow y_{0}$ in $N$. Passing to a subsequence if necessary, we may assume that $q_{j} \in B_{0}$ and $q_{j} \neq q_{j^{\prime}}$ if $j \neq j^{\prime}$. Define $\rho_{y_{0}}: N \rightarrow \mathbb{R}$ by $\rho_{y_{0}}(z)=\operatorname{dist}_{N}\left(y_{0}, z\right)^{2} / 2, z \in N$. Since $r_{0}<\operatorname{inj}_{N}\left(y_{0}\right)$, the function $z \rightarrow \rho_{y_{0}}(z) \in C^{2}$ if $\rho_{y_{0}}(z) \leq r_{0}$. If we let $d_{b_{0}}(x)=\operatorname{dist}_{\mathbb{N}\left(b_{0}\right)}(0, x)$ be the distance to a origin 0 in a simply connected $n$-space form $\mathbb{N}^{n}\left(b_{0}\right)$ of constant sectional curvature $b_{0}$ then by the hessian comparison theorem we obtain

$$
\begin{aligned}
\operatorname{Hess} \rho_{y_{0}}(z)(Y, Y) & \geq \operatorname{Hess} \frac{1}{2} \mathrm{~d}_{b_{0}}\left(p_{0}, p\right)^{2}\left(Y^{\prime}, Y^{\prime}\right) \\
& \geq \sqrt{b_{0}} \cot \left(\sqrt{b_{0}} r_{0}\right)\left|Y^{\prime}\right|^{2} \\
& \geq \frac{1}{2}|Y|^{2},
\end{aligned}
$$

where $\mathrm{d}_{N}\left(y_{0}, z\right)=\mathrm{d}_{b_{0}}\left(p_{0}, p\right) \leq r_{0},|Y|=\left|Y^{\prime}\right|, Y \perp \nabla \rho_{y}$ and $Y^{\prime} \perp \nabla d_{b_{0}}$. We need part of the following result that might have interest in its own.

Lemma 7.3. Let $r \leq r_{0} / 8$. Then

i. For each $x \in \varphi^{-1}\left(B_{0}\right)$ we have $\operatorname{inj}_{M}(x)>r_{0}$.

ii. Let $U_{j}$ be a connected component of $\varphi^{-1}\left(\overline{B_{4 r}^{N}\left(y_{0}\right)}\right)$ containing $q_{j}$, then

$$
\operatorname{dist}_{N}\left(\varphi\left(z_{1}\right), \varphi\left(z_{2}\right)\right) \leq \operatorname{dist}_{M}\left(z_{1}, z_{2}\right) \leq 2 \operatorname{dist}_{N}\left(\varphi\left(z_{1}\right), \varphi\left(z_{2}\right)\right), \forall z_{1}, z_{2} \in U
$$

Thus the map $\varphi_{\mid U_{j}}: U_{j} \rightarrow N$ is an embedding.

iii. Take $x_{j} \in U_{j}$ such that $\operatorname{dist}_{N}\left(y_{0}, \varphi\left(x_{j}\right)\right)=\operatorname{dist}_{N}\left(y_{0}, \varphi\left(U_{j}\right)\right)$. If $j$ is large enough then $B_{3 r}^{M}\left(x_{j}\right) \subset U_{j} \subset B_{10 r}^{M}\left(x_{j}\right)$.

Proof. Let $x \in \varphi^{-1}\left(B_{0}\right)$. Suppose that $\operatorname{dist}_{M}\left(x, \operatorname{cut}_{M}(x)\right)<r_{0}$. Let $z \in \operatorname{cut}_{M}(x)$ such that $\operatorname{dist}_{M}(x, z)=\operatorname{dist}_{M}\left(x, \operatorname{cut}_{M}(x)\right)$. By (127), $z$ is not conjugated to $x$, thus, there are two distinct minimal geodesics $\gamma_{1}$ and $\gamma_{2}$ joining $x$ to $z$, making a geodesic loop $\gamma=\gamma_{1} \cup \gamma_{2}$ based at $x$, [37, Lemma 5.6]. Since $r_{0}>\operatorname{dist}_{M}(x, z) \geq \operatorname{dist}_{N}(\varphi(x), \varphi(z))$, the closed curve $\varphi(\gamma)$ is the region in $N$ where $\rho_{y_{0}}$ is $C^{2}$. The function $h(s)=\rho_{y_{0}}(\varphi(\gamma(t)))$ has a maximum at $s=\operatorname{inj}_{M}(x)$, however

$$
\begin{aligned}
h^{\prime \prime}(s) & =\nabla \mathrm{d} \rho_{y_{0}}\left(d \varphi \gamma^{\prime}, \mathrm{d} \varphi \gamma^{\prime}\right)+\left\langle\nabla \rho_{y_{0}}, \alpha\left(\gamma^{\prime}, \gamma^{\prime}\right)\right\rangle \\
& \geq 1 / 2-r_{0} \Lambda_{0} \\
& \geq 1 / 4, \quad 0 \leq s \leq 2 \operatorname{inj}_{M}(x) .
\end{aligned}
$$

This contradiction proves item $(i)$. To prove $(i i)$, let $U_{j} \subset \varphi^{-1}\left(\overline{B_{4 r}^{N}\left(y_{0}\right)}\right)$ be a connected component containing $q_{j}$. Let $z_{1}, z_{2} \in U_{j}$ and $y_{1}=\varphi\left(z_{1}\right)$ and $y_{2}=\varphi\left(z_{2}\right)$. Let $\gamma(s)$, $s \in\left[0, \operatorname{dist}_{M}\left(z_{1}, z_{2}\right)\right]$ be a minimal geodesic in $M$ joining $z_{1}$ to $z_{2}$. We may assume without loss of generality that $\operatorname{dist}_{N}\left(y_{0}, y_{1}\right) \leq \operatorname{dist}_{N}\left(y_{0}, y_{2}\right)$. Observe that we have $\rho_{y_{0}}(\varphi(\gamma(s))) \leq$ $\rho_{y_{0}}\left(y_{2}\right)$ for all $s$. Otherwise, $s \mapsto \rho_{y_{0}}(\varphi(\gamma(s)))$ has a maximum at some interior point $s_{0} \in\left(0, \operatorname{dist}_{M}\left(z_{1}, z_{2}\right)\right)$ and $\operatorname{dist}_{N}\left(y_{0}, \varphi_{\iota}\left(\gamma\left(s_{0}\right)\right)\right)<r_{0}$. Taking the second derivative at this point of maximum and we get a contradiction, as above, and that proves our assertion. Moreover, $s \mapsto \rho_{y_{1}}(\varphi(\gamma(s)))$ is of class at least $C^{2}$. It is clear that $\left(\rho_{y_{1}}\left(\varphi_{\iota}(\gamma(s))\right)\right)^{\prime \prime} \geq 1 / 4$ 
for all $s \in\left[0, t=\operatorname{dist}_{M}\left(z_{1}, z_{2}\right)\right]$. Then

$$
\begin{aligned}
\frac{\operatorname{dist}_{N}^{2}\left(y_{1}, y_{2}\right)}{2}= & \rho_{y_{1}}(\varphi(\gamma(t))) \\
= & \rho_{y_{1}}(\varphi(\gamma(0)))+\left.t \rho_{y_{1}}(\varphi(\gamma(s)))^{\prime}\right|_{s=0} \\
& +\int_{0}^{1}(1-s)\left(\rho_{y_{1}}(\varphi(\gamma(s t)))\right)^{\prime \prime} \mathrm{d} s \\
\geq & \frac{t^{2}}{4} \int_{0}^{1}(1-s) \mathrm{d} s \\
= & \frac{t^{2}}{8} .
\end{aligned}
$$

It follows that $\operatorname{dist}_{M}\left(z_{1}, z_{2}\right) \leq 2 \operatorname{dist}_{N}\left(\varphi\left(z_{1}\right), \varphi\left(z_{2}\right)\right)$. To prove item iii. pick $x_{j} \in U_{j}$ such that $\operatorname{dist}_{N}\left(y_{0}, \varphi\left(x_{j}\right)\right)=\operatorname{dist}_{N}\left(y_{0}, \varphi\left(U_{j}\right)\right)$. We may choose $j$ large enough so that $\operatorname{dist}_{N}\left(y_{0}, \varphi\left(x_{j}\right)\right)<r$. Let $x \in B_{3 r}^{M}\left(x_{j}\right)$. Then

$$
\begin{aligned}
\operatorname{dist}_{N}\left(\varphi(x), y_{0}\right) & \leq \operatorname{dist}_{N}\left(\varphi(x), \varphi\left(x_{j}\right)\right)+\operatorname{dist}_{N}\left(\varphi\left(x_{j}\right), y\right) \\
& <\operatorname{dist}_{M}\left(x, x_{j}\right)+r \\
& \leq 4 r .
\end{aligned}
$$

On the other hand, let $x \in U$ then we have $\operatorname{dist}_{M}\left(x_{j}, x\right) \leq 2 \operatorname{dist}_{N}\left(\varphi\left(x_{j}\right), \varphi(x)\right)$ and $2 \operatorname{dist}_{N}\left(\varphi\left(x_{j}\right), \varphi(x)\right) \leq 2\left[\operatorname{dist}_{N}\left(\varphi\left(x_{j}\right), y_{0}\right)+\operatorname{dist}_{N}\left(y_{0}, \varphi(x)\right)\right] \leq 10 r$.

By the Lemma 7.3, there exists a sequence $x_{j} \in M$ such that

$$
B_{3 r}^{M}\left(x_{j}\right) \subset U_{j} \subset B_{10 r}^{M}\left(x_{j}\right), \forall j .
$$

Observe that $\operatorname{dist}_{N}\left(q_{j}, y_{0}\right) \geq \operatorname{dist}_{N}\left(\varphi\left(x_{j}\right), y_{0}\right) \rightarrow 0$ as $j \rightarrow \infty$ and then $y_{0} \in \lim \varphi$. Therefore passing to a subsequence we have that $x_{j} \neq x_{j+k}$ for all $k \geq 1$. Recall that the sectional curvatures of $U_{j}$ are bounded below $K_{U_{j}} \geq-b_{0}$. Let $\mathbb{N}^{m}\left(-b_{0}\right)$ the simply connected space form of constant sectional curvature $-b_{0}$. Choose any $\delta \in(0,1)$. By the Bishop-Gromov volume comparison theorem we have

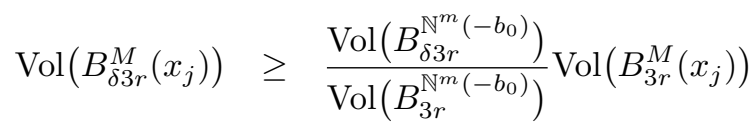

$$
\begin{aligned}
& =C\left(b_{0}, m, \delta, 3 r\right)^{-1} \operatorname{Vol}\left(B_{3 r}^{M}\left(x_{j}\right)\right) \text {. }
\end{aligned}
$$

This shows that $M$ has the ball property with respect to the parameters $\left\{x_{j}\right\}, R=3 r$,

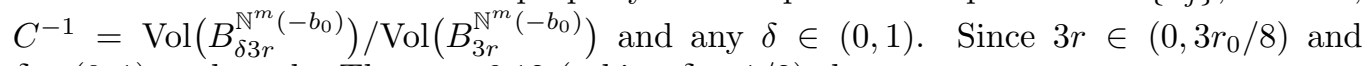
$\delta \in(0,1)$ we have by Theorem 6.12 (taking $\delta=1 / 2)$ that

$$
\left.\inf \sigma_{e s s}(-\Delta) \leq \frac{256}{9 r_{0}^{2}} \cdot \frac{\operatorname{Vol}\left(B_{3 r}^{\mathbb{N}^{m}\left(-b_{0}\right)}\right)}{\operatorname{Vol}\left(B_{\delta 3 r / 2}^{\mathbb{N}^{m}}\left(-b_{0}\right)\right.}\right)
$$

7.3. Proof of Theorem 7.1. To prove Theorem 7.1 we will apply the following proposition derived from the Spectral Theorem, see details in [52, Prop.2], [67, pp. 13-15]. Let $M$ be a Riemannian manifold.

Proposition 7.4. A necessary and sufficient condition for $(\eta-\epsilon, \eta+\epsilon) \cap \sigma_{\mathrm{ess}}(-\Delta) \neq \emptyset$ is that there exists an infinite dimensional subspace $G_{\epsilon} \subset D(-\Delta)$ of the domain of $-\Delta$, for which $\|(\Delta+\eta I) \psi\|_{L^{2}(M)}<\epsilon\|\psi\|_{L^{2}(M)}, \psi \in G_{\epsilon}$. 
To show that $\eta \geq 0$ belongs to $\sigma_{\text {ess }}(-\Delta)$ we need to take a sequence $v_{n} \rightarrow 0$ as $n \rightarrow \infty$ and a sequence of functions $\psi_{n} \in C_{0}^{\infty}(M)$ satisfying $\left\|(\Delta+\eta I) \psi_{n}\right\|_{L^{2}(M)}<v_{n}\left\|\psi_{n}\right\|_{L^{2}(M)}$ with $\operatorname{supp} \psi_{n} \cap \operatorname{supp} \psi_{n^{\prime}}=\emptyset$ if $n \neq n^{\prime}$.

Consider a sequence of compact subsets $K_{n} \subset D_{n}$ with Euclidean width $r_{n} \rightarrow 0$ as $n \rightarrow \infty$ and the set of constants $c_{n}$ satisfying (120) in Jorge-Xavier's or RosenbergToubiana's construction. The induced metric on the minimal surface is conformal to the Euclidean metric $|\mathrm{d} z|^{2}$ on the disk $\mathbb{D}$, this is $\mathrm{d} s^{2}=\lambda^{2}|\mathrm{~d} z|^{2}$. Set $\lambda_{n}=\sup _{K_{n}} \lambda(z)$ and $\zeta_{n}=\lambda_{n} /\left(\inf _{K_{n}} \lambda(z)\right)$ so that $\lambda_{n} / \zeta_{n} \leq \lambda \leq \lambda_{n}$ in $K_{n}$. Let $I_{n}$ be the segment of the real axis that crosses $K_{n}$. The length $\ell_{\mathrm{d} s^{2}}\left(I_{n}\right)$ of $I_{n}$ in the metric $\mathrm{d} s^{2}$ has the following lower and upper bound

$$
\frac{\lambda_{n} r_{n}}{\zeta_{n}} \leq \ell_{\mathrm{d} s^{2}}\left(I_{n}\right) \leq \lambda_{n} r_{n} .
$$

Let $p_{n}$ be the center of the $I_{n}$ and denote by $B_{t}^{\mathrm{ds}^{2}}\left(p_{n}\right)$ and $B_{t}^{|\mathrm{d} z|^{2}}\left(p_{n}\right)$ the geodesic balls of radius $t$ and center $p_{n}$ with respect to the metrics $\mathrm{d} s^{2}$ and $|\mathrm{d} z|^{2}$ respectively. Denote by $\Delta^{|\mathrm{d} z|^{2}}$ and by $\mathrm{d} x$, respectively the Laplace operator and the Lebesgue measure of $\mathbb{R}^{2}$ with respect to the metric $|\mathrm{d} z|^{2}$ and denote by $\Delta^{\mathrm{d} s^{2}}$ and by $\lambda^{2} \mathrm{~d} x$ the Laplace operator and the Riemannian measure on $M$ with respect to the metric $\mathrm{d} s^{2}$. The Laplace operators $\Delta^{|\mathrm{d} z|^{2}}$ and $\Delta^{\mathrm{d} s^{2}}$ are related, on $\mathbb{D}$, by $\Delta^{\mathrm{d} s^{2}}=\frac{1}{\lambda^{2}} \Delta^{|\mathrm{d} z|^{2}}$. Given $\eta>0$ and $f \in C_{0}^{\infty}\left(B_{r_{n}}^{|\mathrm{d} z|^{2}}\left(p_{n}\right)\right)$ be a smooth function with compact support in $B_{r_{n}}^{|\mathrm{d} z|^{2}}\left(p_{n}\right) \subset K_{n}$ to be chosen later. We have that

$$
\begin{aligned}
& \left\|\Delta^{\mathrm{d} s^{2}} f+\eta f\right\|_{L^{2}(M)}^{2}=\int_{\left.B_{r_{n}}^{\mathrm{d} z}\right|^{2}\left(p_{n}\right)}\left(\frac{1}{\lambda^{2}} \Delta^{|\mathrm{d} z|^{2}} f+\eta f\right)^{2} \lambda^{2} \mathrm{~d} x \\
& =\int_{B_{r_{n}}^{|\mathrm{d} z|^{2}}\left(p_{n}\right)} \frac{1}{\lambda^{2}}\left(\Delta^{|d z|^{2}} f\right)^{2} \mathrm{~d} x+\eta^{2} \int_{B_{r_{n}}^{|\mathrm{d} z|^{2}}\left(p_{n}\right)} f^{2} \lambda^{2} \mathrm{~d} x \\
& +2 \eta \int_{B_{r_{n}}^{|\mathrm{d} z|^{2}}\left(p_{n}\right)} f \Delta^{|\mathrm{d} z|^{2}} f \mathrm{~d} x \\
& \leq \int_{B_{r_{n}}^{|\mathrm{d} z|^{2}}\left(p_{n}\right)} \frac{\epsilon_{n}^{2}}{\lambda_{n}^{2}}\left(\Delta^{|\mathrm{d} z|^{2}} f\right)^{2} \mathrm{~d} x \\
& +\eta^{2} \zeta_{n}^{2} \int_{B_{r_{n}}^{|\mathrm{d} z|^{2}}\left(p_{n}\right)} f^{2} \lambda_{n}^{2} \mathrm{~d} x \\
& +2 \eta \zeta_{n}^{2} \int_{B_{r_{n}}^{|\mathrm{d} z|^{2}}\left(p_{n}\right)} f \Delta^{|\mathrm{d} z|^{2}} f \mathrm{~d} x \\
& +2 \eta\left(\zeta_{n}^{2}-1\right) \int_{B_{r_{n}}^{|\mathrm{d} z|^{2}\left(p_{n}\right)}}\left|\nabla^{|\mathrm{d} z|^{2}} f\right|^{2} \mathrm{~d} x \\
& =\zeta_{n}^{2} \int_{B_{r_{n}}^{|\mathrm{d} z|^{2}}\left(p_{n}\right)}\left(\frac{1}{\lambda_{n}^{2}} \Delta^{|\mathrm{d} z|^{2}} f+\eta f\right)^{2} \lambda_{n}^{2} \mathrm{~d} x \\
& +2 \eta\left(\zeta_{n}^{2}-1\right) \int_{B_{r_{n}}^{|\mathrm{d} z|^{2}}\left(p_{n}\right)}\left|\nabla^{|\mathrm{d} z|^{2}} f\right|^{2} \mathrm{~d} x .
\end{aligned}
$$

Let us consider the ball $B_{\lambda_{n} r_{n}}^{|\mathrm{d} z|^{2}}\left(p_{n}\right)=p_{n}+B_{\lambda_{n} r_{n}}^{|\mathrm{d} z|^{2}}(0) \subset \mathbb{R}^{2}$ of radius $\lambda_{n} r_{n}$ and center $p_{n}$ and the map $\xi: B_{\lambda_{n} r_{n}}^{|\mathrm{d} z|^{2}}\left(p_{n}\right) \rightarrow B_{r_{n}}^{|\mathrm{d} z|^{2}}\left(p_{n}\right)$ given by $\xi\left(p_{n}+x\right)=p_{n}+x / \lambda_{n}$ and define $h: B_{\lambda_{n} r_{n}}^{|\mathrm{d} z|^{2}}\left(p_{n}\right) \rightarrow \mathbb{R}$ by $h=f \circ \xi$. We have that $\Delta^{|\mathrm{d} z|^{2}} h=\Delta^{|\mathrm{d} z|^{2}} f(\xi) / \lambda_{n}^{2}$ and the Jacobian $J(\xi)(x)=1 / \lambda_{n}^{2}$. Making the change of variables $x=\xi(y)$ we have that

$$
\text { - } \int_{B_{r_{n}}^{|\mathrm{d} z|^{2}}\left(p_{n}\right)}\left(\frac{1}{\lambda_{n}^{2}} \Delta^{|\mathrm{d} z|^{2}} f+\eta f\right)^{2} \lambda_{n}^{2} \mathrm{~d} x=\int_{B_{\lambda_{n} r_{n}}^{\left.\mid \mathrm{d} z p_{n}\right)}}\left(\Delta^{|\mathrm{d} z|^{2}} h+\eta h\right)^{2} \mathrm{~d} x .
$$


- $\int_{B_{r_{n}}^{|\mathrm{d} z|^{2}}\left(p_{n}\right)}\left|\nabla^{|\mathrm{d} z|^{2}} f\right|^{2} \mathrm{~d} x=\left.\left.\int_{B_{\lambda_{n} r_{n}}^{|\mathrm{d} z|_{n}^{2}}\left(p_{n}\right)}|\nabla| \mathrm{d} z\right|^{2} h\right|^{2} \mathrm{~d} x$.

Thus from (130) and the change of variable above we have the following inequality

$$
\begin{aligned}
& \left\|\Delta^{\mathrm{d} s^{2}} f+\eta f\right\|_{L^{2}(M)} \leq \zeta_{n}\left\|\Delta^{|\mathrm{d} z|^{2}} h+\eta h\right\|_{L^{2}\left(B_{\lambda_{n} r_{n}}^{|\mathrm{d} z|^{2}}\left(p_{n}\right)\right)} \\
& +\sqrt{2 \eta\left(\zeta_{n}^{2}-1\right)}\left\|\nabla^{|\mathrm{d} z|^{2}} h\right\|_{L^{2}\left(B_{\lambda_{n} r_{n}}^{|\mathrm{d} z|^{2}}\left(p_{n}\right)\right)},
\end{aligned}
$$

where $f: B_{r_{n}}^{|\mathrm{d} z|^{2}}\left(p_{n}\right) \subset K_{n} \rightarrow \mathbb{R}, h=f \circ \xi: B_{\lambda_{n} r_{n}}^{|\mathrm{d} z|^{2}}\left(p_{n}\right) \rightarrow \mathbb{R}$ defined by $h\left(p_{n}+x\right)=$ $f\left(p_{n}+x / \lambda_{n}\right)$. Observe that $f=h \circ \xi^{-1}: B_{r_{n}}^{|\mathrm{d} z|^{2}}\left(p_{n}\right) \rightarrow \mathbb{R}$ so that $f\left(p_{n}+x\right)=h\left(p_{n}+\lambda_{n} x\right)$, $x \in B_{r_{n}}^{|\mathrm{d} z|^{2}}(0)$.

Therefore, given $h \in C_{0}^{\infty}\left(B_{\lambda_{n} r_{n}}^{|\mathrm{d} z|^{2}}\left(p_{n}\right)\right)$ we obtain $f \in C_{0}^{\infty}\left(B_{r_{n}}^{|\mathrm{d} z|^{2}}\left(p_{n}\right)\right)$ and vice-versa, satisfying inequality (131).

Since $\sigma\left(-\Delta^{|\mathrm{d} z|^{2}}\right)=\sigma_{\text {ess }}\left(-\Delta^{|\mathrm{d} z|^{2}}\right)=[0, \infty)$, given a positive number $\eta>0$ we have that $\eta \in \sigma_{\text {ess }}\left(-\Delta^{|\mathrm{d} z|^{2}}\right)$. Therefore for each $\delta>0$ there exists, (by Proposition 7.4), $h \in C_{0}^{\infty}\left(\mathbb{R}^{2}\right)$ such that

$$
\left\|\Delta^{|\mathrm{d} z|^{2}} h+\eta h\right\|_{L^{2}\left(\mathbb{R}^{2}\right)}<\delta\|h\|_{L^{2}\left(\mathbb{R}^{2}\right)} .
$$

Suppose that $\limsup _{n \rightarrow \infty} r_{n} \lambda_{n}=\infty$. Then there exists $n_{0}$ such that for all $n \geq n_{0}$ the ball $B_{\lambda_{n} r_{n}}^{|\mathrm{d} z|^{2}}\left(p_{n}\right)$ contains the support of $h$ since for large $n$ we have $1 \leq e_{n}<2$ and the length $\ell_{d s^{2}}\left(I_{n}\right) \geq \lambda_{n} r_{n} / \zeta_{n} \rightarrow \infty$. For this function $h \in C_{0}^{\infty}\left(B_{\lambda_{n} r_{n}}^{|\mathrm{d} z|^{2}}\left(p_{n}\right)\right)$ we have

- $\int_{B_{\lambda_{n} r_{n}}^{|\mathrm{d} z|_{n}^{2}\left(p_{n}\right)}}\left|\nabla^{|\mathrm{d} z|^{2}} h\right|^{2} \mathrm{~d} x \leq \mu_{1}(n) \int_{B_{\lambda_{n} r_{n}}^{|\mathrm{d} z|^{2}}\left(p_{n}\right)} h^{2} \mathrm{~d} y$, where $\mu_{1}(n)$ is the first Dirichlet eigenvalue of the ball $B_{\lambda_{n} r_{n}}^{|\mathrm{d} z|^{2}}\left(p_{n}\right)$.

- Letting $f\left(p_{n}+x\right)=h\left(p_{n}+\lambda_{n} x\right) \in C_{0}^{\infty}\left(B_{r_{n}}^{|\mathrm{d} z|^{2}}\left(p_{n}\right)\right)$ we have

$$
\begin{aligned}
\int_{B_{\lambda_{n} r_{n}}^{|\mathrm{d} z|^{2}\left(p_{n}\right)}} h^{2} \mathrm{~d} y & =\int_{B_{r_{n}}^{|\mathrm{d} z|^{2}\left(p_{n}\right)}} \lambda_{n}^{2} f^{2} \mathrm{~d} x \\
& \leq 4 \int_{B_{r_{n}}^{|\mathrm{d} z|^{2}\left(p_{n}\right)}} f^{2} \lambda^{2} \mathrm{~d} x \\
& =4\|f\|_{L^{2}(M)}^{2},
\end{aligned}
$$

since $\lambda_{n} \leq 2 \lambda$.

- Putting together these information we have

$$
\int_{B_{\lambda_{n} r_{n}}^{|\mathrm{d} z|^{2}\left(p_{n}\right)}}\left|\nabla^{|\mathrm{d} z|^{2}} h\right|^{2} \mathrm{~d} x \leq 4\|f\|_{L^{2}(M)}^{2} .
$$

From inequality (131) we have then

$$
\left\|\Delta^{d s^{2}} f+\eta f\right\|_{L^{2}(M)} \leq\left(2 \zeta_{n} \delta+2 \sqrt{2 \eta\left(\zeta_{n}^{2}-1\right) \mu_{1}(n)}\right)\|f\|_{L^{2}(M)} .
$$

We are ready to conclude that each $\eta>0$ belongs to $\sigma_{\text {ess }}\left(-\Delta^{\mathrm{d} s^{2}}\right)$. Consider a sequence of positive numbers $v_{i} \rightarrow 0$. For each $i$, choose $n$ such that $2 \sqrt{2 \eta\left(\epsilon_{n_{i}}^{2}-1\right) \mu_{1}\left(n_{i}\right)}<v_{i} / 2$. This $n$ exists since $\mu_{1}(n)=\lambda_{1}\left(B_{\lambda_{n} r_{n}}^{|\mathrm{d} z|^{2}}\left(p_{n}\right)\right)=c /\left(\lambda_{n} r_{n}\right)^{2} \rightarrow 0$ and $\epsilon_{n} \rightarrow 1$ as $n \rightarrow \infty$. Take $\delta<v_{i} / 4$ and choose $h_{i} \in C_{0}^{\infty}\left(\mathbb{R}^{2}\right)$ such that (131) holds and choosing $n_{i}$ large enough so that $\operatorname{supp} h_{i} \subset B_{\lambda_{n_{i}} r_{n_{i}}}^{|\mathrm{d} z|^{2}}\left(p_{n}\right)$. Then the function $f_{i}$ associated to $h_{i}$ satisfies

$$
\left\|\Delta^{d s^{2}} f_{i}+\eta f_{i}\right\|_{L^{2}(M)}<v_{i}\left\|f_{i}\right\|_{L^{2}(M)} .
$$


It is clear that we can choose the family $h_{i}$ with support in different balls. All that shows that $\eta \in \sigma_{\text {ess }}\left(-\Delta^{\mathrm{d} s^{2}}\right)$. To finish the proof of Theorems 7.1 we need to address the case that $\lim \sup r_{n} \lambda_{n}>0$. Observe that in $K_{n}$ we have that

$$
\frac{\lambda_{n}}{\zeta_{n}} \leq \lambda \leq \lambda_{n}
$$

This implies that, in $K_{n}$,

$$
\left(\frac{\lambda_{n}}{\zeta_{n}}\right)^{2}|\mathrm{~d} z|^{2} \leq \mathrm{d} s^{2} \leq \lambda_{n}^{2}|\mathrm{~d} z|^{2} .
$$

From this point on, is easy to see that $\left(\mathbb{D}, \mathrm{d} s^{2}\right)$ or $\left(\mathbb{A}(1 / c, c), \mathrm{d} s^{2}\right)$ has the ball property, see details in the application the subsection 7.1. Thus $\sigma_{\text {ess }}\left(\mathrm{d} s^{2}\right) \neq \emptyset$. This finishes the proof of Theorem 7.1 .

7.4. Immersed submanifolds, II: density and spectrum. Hereafter, we will consider proper (hence, complete), minimal immersions $\varphi: M^{m} \rightarrow \mathbb{N}_{\kappa}^{n}$ in a space form $\mathbb{N}_{\kappa}^{n}$ of curvature $-\kappa \leq 0$. Our main concern is to describe a characterization of the whole $\sigma(M)$, due to [91], which is free from curvature or topological conditions on $M$. It is known by [41] and [22], see Theorem 2.32, that for a minimal immersion $\varphi: M^{m} \rightarrow \mathbb{N}_{\kappa}^{n}$ the fundamental tone $\lambda^{*}(M)=\inf \sigma(M)$ is at least that of $\mathbb{N}_{\kappa}^{m}$, i.e.,

$$
\inf \sigma(M) \geq \frac{(m-1)^{2} \kappa}{4}
$$

Moreover, as a corollary of [88] and [18], [16], if the second fundamental form II satisfies the decay estimate

$$
\begin{array}{ll}
\lim _{\rho(x) \rightarrow+\infty} \rho(x)|\operatorname{II}(x)|=0 & \text { if } \kappa=0 \\
\lim _{\rho(x) \rightarrow+\infty}|\operatorname{II}(x)|=0 & \text { if } \kappa>0
\end{array}
$$

( $\rho(x)$ being the intrinsic distance with respect to some fixed origin $o \in M$ ), then $M$ has the same spectrum that a totally geodesic submanifold $\mathbb{M}_{\kappa}^{m} \subset \mathbb{N}_{\kappa}^{n}$, that is,

$$
\sigma(M)=\left[\frac{(m-1)^{2} \kappa}{4},+\infty\right)
$$

According to [5], [108], (135) is ensured when $M$ has finite total curvature, that is, when

$$
\int_{M}|\mathrm{III}|^{m}<+\infty
$$

Remark 7.5. A characterization of the essential spectrum, similar to (136), also holds for submanifolds of the hyperbolic space $\mathbb{H}_{\kappa}^{n}$ with constant (normalized) mean curvature $H<\sqrt{\kappa}$. There, condition (137) is replaced by the finiteness of the $L^{m}$-norm of the traceless second fundamental form. For deepening, see [31].

Since condition (135) is a binding requirement, needing a pointwise control of the second fundamental form, the search for more manageable conditions lead the authors of [91] to investigate the growth of the density function

$$
\Theta(r) \doteq \frac{\operatorname{vol}\left(M \cap B_{r}\right)}{V_{\kappa}(r)},
$$

where $B_{r}$ indicates a geodesic ball of radius $r$ in $\mathbb{N}_{\kappa}^{n}$ centered at some fixed $\bar{o} \in \mathbb{N}_{\kappa}^{n}$, and $V_{\kappa}(r)$ is the volume of a geodesic ball of radius $r$ in $\mathbb{N}_{\kappa}^{m}$. By classical result (see $[129,6]$ ), $\Theta(r)$ is monotone increasing, and we will say that $M$ has finite density if

$$
\Theta(+\infty) \doteq \lim _{r \rightarrow+\infty} \Theta(r)<+\infty .
$$


In the ambient space $\mathbb{R}^{n}$, it is known by Chern-Osserman's inequalities that (137) imply the finiteness of $\Theta(+\infty)([45,46,5])$. By [91], the same is true in the hyperbolic space $\mathbb{H}_{\kappa}^{n}$, although the Chern-Osserman's inequality was known just for surfaces in $\mathbb{H}_{\kappa}^{n}$, see $[35,36]$.

The main result in [91] characterizes $\sigma(M)$ when the density of $M$ grows subexponentially (respectively, sub-polynomially) along a sequence. Condition (139) below is very much in the spirit of a classical growth requirement due to R. Brooks [29] and Y. Higuchi [79] to bound from above the infimum of the essential spectrum of $-\Delta$. We feel remarkable that just a volume growth condition along a sequence can characterize the whole spectrum. For this to hold, minimality enters in a crucial and subtle way via (an improved version of) the monotonicity formula. Note that, by standard estimates, (139) below is weaker than (135).

Theorem 7.6. [Lima-Mari-Montenegro-Vieira [91]] Let $\varphi: M^{m} \rightarrow \mathbb{N}_{\kappa}^{n}$ be a minimal properly immersed submanifold in a space form of curvature $-\kappa \leq 0$. If either

$$
\begin{array}{ll}
\mathbb{N}_{\kappa}^{n}=\mathbb{H}_{\kappa}^{n} \text { and } & \liminf _{s \rightarrow+\infty} \frac{\log \Theta(s)}{s}=0, \quad \text { or } \\
\mathbb{N}_{\kappa}^{n}=\mathbb{R}^{n} \text { and } & \liminf _{s \rightarrow+\infty} \frac{\log \Theta(s)}{\log s}=0 .
\end{array}
$$

then

$$
\sigma(M)=\left[\frac{(m-1)^{2} \kappa}{4},+\infty\right) .
$$

The above theorem is well suited for minimal submanifolds constructed via Geometric Measure Theory since, typically, their existence is guaranteed by controlling the density function $\Theta(r)$. As an important example, Theorem 7.6 applies to all solutions of Plateau's problem at infinity $M^{m} \rightarrow \mathbb{H}_{\kappa}^{n}$ constructed in [6], provided that they are smooth. Indeed, because of their construction, $\Theta(+\infty)<+\infty$ (see [6], part [A] at p. 485) and they are proper (it can also be deduced as a consequence of $\Theta(+\infty)<+\infty$, see [91]). By standard regularity theory, smoothness of $M^{m}$ is automatic if $m \leq 6$.

Corollary 7.7. Let $\Sigma \subset \partial_{\infty} \mathbb{H}_{\kappa}^{n}$ be a closed, integral $(m-1)$ current in the boundary at infinity of $\mathbb{H}_{\kappa}^{n}$ such that, for some neighbourhood $U \subset \mathbb{H}_{k}^{n}$ of $\operatorname{supp}(\Sigma), \Sigma$ does not bound in $U$, and let $M^{m} \hookrightarrow \mathbb{H}_{\kappa}^{n}$ be the solution of Plateau's problem at infinity constructed in [6] for $\Sigma$. If $M$ is smooth, then (140) holds.

An interesting fact of Corollary 7.7 is that $M$ is not required to be regular up to $\partial_{\infty} \mathbb{H}_{\kappa}^{n}$, in particular it might have infinite total curvature. In this respect, we observe that if $M$ be $C^{2}$ up to $\partial_{\infty} \mathbb{H}_{\kappa}^{n}$, then $M$ would have finite total curvature. By deep regularity results, this is the case if, for instance, $M^{m} \rightarrow \mathbb{H}_{\kappa}^{m+1}$ is a smooth hypersurface that solves Plateau's problem for $\Sigma$, and $\Sigma$ is a $C^{2, \alpha}$ (for $\alpha>0$ ), embedded compact hypersurface of $\partial_{\infty} \mathbb{H}_{\kappa}^{m+1}$. See Appendix 1 in [91] for details.

We sketch the strategy of the proof of Theorem 7.6. In view of (134), it is enough to show that each $\lambda>(m-1)^{2} \kappa / 4$ lies in $\sigma(M)$. To construct the sequence as in Lemma 6.1 , a key step is to couple the volume growth requirement (139) with a sharpened form of the monotonicity formula for minimal submanifolds, which improves on the classical ones in $[129,6]$.

\section{Notation}

We denote with $\nabla$, Hess, $\Delta$ the connection, the Riemannian Hessian and the LaplaceBeltrami operator on $M^{m}$, while quantities related to $\mathbb{N}_{\kappa}^{n}$ will be marked with a bar. For instance, $\bar{\nabla}, \overline{\text { dist }}, \overline{\text { Hess }}$ will identify the connection, the distance function and the Hessian in $\mathbb{N}_{\kappa}^{n}$. Let $\bar{\rho}(x)=\overline{\operatorname{dist}}(x, \bar{o})$ be the distance function from a fixed origin $\bar{o}$, and let 
$r=\bar{\rho} \circ \varphi: M \rightarrow \mathbb{R}$ be its restriction to $M$. We will indicate with $\Gamma_{s}$ the extrinsic geodesic spheres restricted to $M: \Gamma_{s} \doteq\{x \in M ; r(x)=s\}$.

For $\kappa \geq 0$, let $v_{\kappa}, V_{\kappa}$ denote the volume function, respectively, of geodesic spheres and balls in the space form of sectional curvature $-\kappa$ and dimension $m$, i.e.,

$$
v_{\kappa}(s)=\omega_{m-1} S_{\kappa}(s)^{m-1}, \quad V_{\kappa}(s)=\int_{0}^{s} v_{\kappa}(\sigma) \mathrm{d} \sigma,
$$

where $\omega_{m-1}$ is the volume of the unit sphere $\mathbb{S}^{m-1}$. We define the $f l u x J(s)$ of $\nabla r$ over the extrinsic sphere $\Gamma_{s}$ :

$$
J(s) \doteq \frac{1}{v_{\kappa}(s)} \int_{\Gamma_{s}}|\nabla r|
$$

We first observe that minimal submanifolds of space forms of non-positive curvature enjoy the following corollary of the coarea's formula: for each $f \in L^{1}(\{t \leq r \leq s\})$,

$$
\int_{\{t \leq r \leq s\}} f \mathrm{~d} x=\int_{t}^{s}\left[\int_{\Gamma_{\sigma}} \frac{f}{|\nabla r|}\right] \mathrm{d} \sigma .
$$

This depends on a transversality lemma which follows from the fact that spheres in $\mathbb{N}_{k}^{n}$ have definite second fundamental form, see [91] for details. The basic lemma is the following improvement of the monotonicity formula:

Proposition 7.8 (The monotonicity formulae). If $\varphi: M^{m} \rightarrow \mathbb{N}_{\kappa}^{n}$ is minimal, proper and $-\kappa \leq 0$, then $J(s)$ coincides a.e. with a non-decreasing function, and $J(s) \geq \Theta(s)$ a.e. on $\mathbb{R}^{+}$. As a consequence, $\Theta(s)$ is non-decreasing.

Remark 7.9. To the best of our knowledge, the monotonicity of $J(s)$ has first been shown, in the Euclidean setting, in a paper by V. Tkachev [133].

Proof. Observe that $J(s)$ coincides, for regular values of $r(x)$, with the absolutely continuous function

$$
\bar{J}(s)=\frac{1}{v(r)} \int_{\{r \leq s\}} \Delta r .
$$

Applying (143) with $f=\Delta r$ we get

$$
v_{\kappa}(s) \bar{J}(s) \doteq \int_{\{r \leq s\}} \Delta r \equiv \int_{0}^{s}\left[\int_{\Gamma_{\sigma}} \frac{\Delta r}{|\nabla r|}\right] \mathrm{d} \sigma
$$

Consider

$$
f(s)=\int_{0}^{s} \frac{V_{\kappa}(\sigma)}{v_{\kappa}(\sigma)} \mathrm{d} \sigma=\int_{0}^{s} \frac{1}{v_{\kappa}(\sigma)}\left[\int_{0}^{\sigma} v_{\kappa}(\tau) \mathrm{d} \tau\right] \mathrm{d} \sigma
$$

which is a $C^{2}$ solution of

$$
f^{\prime \prime}+\frac{v_{\kappa}^{\prime}}{v_{\kappa}} f^{\prime}=1 \quad \text { on } \mathbb{R}^{+}, \quad f(0)=0, \quad f^{\prime}(0)=0,
$$

and define $\psi(x)=f(r(x)) \in C^{2}(M)$. A computation usign the Hessian comparison theorem shows that $\Delta \psi \geq 1$ on $M$. Integrating on $\{r \in[t, s]\}, t, s$ regular values, and using the divergence theorem we get

$$
\frac{V_{\kappa}(s)}{v_{\kappa}(s)} \int_{\Gamma_{s}}|\nabla r|-\frac{V_{\kappa}(t)}{v_{\kappa}(t)} \int_{\Gamma_{t}}|\nabla r| \geq \operatorname{vol}(\{t \leq r \leq s\}) .
$$

By the definition of $J(s), \bar{J}(s)$ and $\Theta(s)$, rearranging we get

$$
V_{\kappa}(s)[\bar{J}(s)-\Theta(s)] \geq V_{\kappa}(t)[\bar{J}(t)-\Theta(t)] .
$$

Since all the quantities involved are continuous, the above relation extends to all $t, s \in \mathbb{R}^{+}$, which proves the monotonicity of $V_{\kappa}[\bar{J}-\Theta]$. Letting $t \rightarrow 0$ we then deduce that $\bar{J}(s) \geq \Theta(s)$ 
on $\mathbb{R}^{+}$, as required. Using that $v(s) J(s) \leq(V(s) \Theta(s))^{\prime}$ for regular $s$, we deduce that $\Theta(s)$ is non-decreasing. The monotonicity of $\bar{J}$ is not used here, and we refer to [91] for details.

\section{Proof of Theorem 1}

Let $M^{m} \rightarrow \mathbb{N}_{\kappa}^{n}$ be minimal, proper. By [41] and [22], the bottom of $\sigma(M)$ satisfies

$$
\inf \sigma(M) \geq \frac{(m-1)^{2} \kappa}{4} .
$$

To complete the proof of the theorem, since $\sigma(M)$ is closed it is sufficient to show that each $\lambda>(m-1)^{2} \kappa / 4$ lies in $\sigma(M)$.

Set for convenience $\beta \doteq \sqrt{\lambda-(m-1)^{2} \kappa / 4}$ and, for $0 \leq t<s$, let $A_{t, s}$ denote the extrinsic annulus

$$
A_{t, s} \doteq\{x \in M: r(x) \in[t, s]\} .
$$

Define the weighted measure $\mathrm{d} \mu_{\kappa} \doteq v_{\kappa}(r)^{-1} \mathrm{~d} x$ on $\{r \geq 1\}$. Hereafter, we will always restrict to this set. Consider

$$
\psi(s) \doteq \frac{e^{i \beta s}}{\sqrt{v_{\kappa}(s)}}, \quad \text { which solves } \quad \psi^{\prime \prime}+\psi^{\prime} \frac{v_{\kappa}^{\prime}}{v_{\kappa}}+\lambda \psi=a(s) \psi,
$$

where

$$
a(s) \doteq \frac{(m-1)^{2} \kappa}{4}+\frac{1}{4}\left(\frac{v_{\kappa}^{\prime}(s)}{v_{\kappa}(s)}\right)^{2}-\frac{1}{2} \frac{v_{\kappa}^{\prime \prime}(s)}{v_{\kappa}(s)} \rightarrow 0
$$

as $s \rightarrow+\infty$. Fix $R>1$ large such that $\Theta(R)>0$, and $t, s, S$ such that

$$
R+1<t<s<S-1,
$$

and let $\eta \in C_{c}^{\infty}(\mathbb{R})$ be a cut-off function satisfying

$$
\begin{aligned}
& 0 \leq \eta \leq 1, \quad \eta \equiv 0 \text { outside of }(t-1, S), \quad \eta \equiv 1 \quad \text { on }(t, s), \\
& \left|\eta^{\prime}\right|+\left|\eta^{\prime \prime}\right| \leq C_{0} \quad \text { on }[t-1, s], \quad\left|\eta^{\prime}\right|+\left|\eta^{\prime \prime}\right| \leq \frac{C_{0}}{S-s} \quad \text { on }[s, S]
\end{aligned}
$$

for some absolute constant $C_{0}$ (the last relation is possible since $S-s \geq 1$ ). The value $S$ will be chosen later in dependence of $s$. Set $u_{t, s} \doteq \eta(r) \psi(r) \in C_{c}^{\infty}(M)$. Then, by (148),

$$
\begin{aligned}
\Delta u_{t, s}+\lambda u_{t, s}= & \left(\eta^{\prime \prime} \psi+2 \eta^{\prime} \psi^{\prime}+\eta \psi^{\prime \prime}\right)|\nabla r|^{2}+\left(\eta^{\prime} \psi+\eta \psi^{\prime}\right) \Delta r+\lambda \eta \psi \\
= & \left(\eta^{\prime \prime} \psi+2 \eta^{\prime} \psi^{\prime}-\frac{v_{\kappa}^{\prime}}{v_{\kappa}} \eta \psi^{\prime}-\lambda \eta \psi+a \eta \psi\right)\left(|\nabla r|^{2}-1\right)+a \eta \psi \\
& +\left(\eta^{\prime} \psi+\eta \psi^{\prime}\right)\left(\Delta r-\frac{v_{\kappa}^{\prime}}{v_{\kappa}}\right)+\left(\eta^{\prime \prime} \psi+2 \eta^{\prime} \psi^{\prime}+\eta^{\prime} \psi \frac{v_{\kappa}^{\prime}}{v_{\kappa}}\right) .
\end{aligned}
$$

Using that there exists an absolute constant $c$ for which $|\psi|+\left|\psi^{\prime}\right| \leq c / \sqrt{v_{\kappa}}$, the following inequality holds:

$$
\begin{aligned}
\left\|\Delta u_{t, s}+\lambda u_{t, s}\right\|_{2}^{2} \leq & C\left(\int_{A_{t-1, S}}\left[\left(1-|\nabla r|^{2}\right)^{2}+\left(\Delta r-\frac{v_{\kappa}^{\prime}}{v_{\kappa}}\right)^{2}+a(r)^{2}\right] \mathrm{d} \mu_{\kappa}\right. \\
& \left.+\frac{\mu_{\kappa}\left(A_{s, S}\right)}{(S-s)^{2}}+\mu_{\kappa}\left(A_{t-1, t}\right)\right)
\end{aligned}
$$


for some suitable $C$ depending on $c, C_{0}$. Since $\left\|u_{t, s}\right\|_{2}^{2} \geq \mu_{\kappa}\left(A_{t, s}\right)$ and $\left(1-|\nabla r|^{2}\right)^{2} \leq$ $1-|\nabla r|^{2}$, we obtain

$$
\begin{aligned}
\frac{\left\|\Delta u_{t, s}+\lambda u_{t, s}\right\|_{2}^{2}}{\left\|u_{t, s}\right\|_{2}^{2}} \leq & C\left(\frac{1}{\mu_{\kappa}\left(A_{t, s}\right)} \int_{A_{t-1, S}}\left[1-|\nabla r|^{2}+\left(\Delta r-\frac{v_{\kappa}^{\prime}}{v_{\kappa}}\right)^{2}+a(r)^{2}\right] \mathrm{d} \mu_{\kappa}\right. \\
& \left.+\frac{1}{(S-s)^{2}} \frac{\mu_{\kappa}\left(A_{s, S}\right)}{\mu_{\kappa}\left(A_{t, s}\right)}+\frac{\mu_{\kappa}\left(A_{t-1, t}\right)}{\mu_{\kappa}\left(A_{t, s}\right)}\right)
\end{aligned}
$$

Next, by the expression of $\Delta r$ we deduce

$$
\begin{aligned}
\left(\Delta r-\frac{v_{\kappa}^{\prime}}{v_{\kappa}}\right)^{2}+1-|\nabla r|^{2}+a(r)^{2} \leq & {\left[\frac{v_{\kappa}^{\prime}}{v_{\kappa}} \frac{1-|\nabla r|^{2}}{m-1}\right]^{2} } \\
& +1-|\nabla r|^{2}+a(r)^{2} \\
\leq & C\left(1-|\nabla r|^{2}+a(r)^{2}\right)
\end{aligned}
$$

Set

$$
F(t) \doteq \sup _{\sigma \in[t-1,+\infty)}\left[a(\sigma)^{2}\right]
$$

and note that $F(t) \rightarrow 0$ monotonically as $t \rightarrow+\infty$. Integrating (151) we get the existence of $C>0$ independent of $s, t$ such that

$$
\begin{aligned}
& \int_{A_{t-1, S}} {\left[\left(\Delta r-\frac{v_{\kappa}^{\prime}}{v_{\kappa}}\right)^{2}+1-|\nabla r|^{2}+a(r)^{2}\right] \mathrm{d} \mu_{\kappa} } \\
& \leq C\left(F(t) \int_{A_{t-1, S}} \frac{1}{v_{\kappa}(r)}+\int_{A_{t-1, S}} \frac{1-|\nabla r|^{2}}{v_{\kappa}(r)}\right) .
\end{aligned}
$$

Using the coarea's formula, for each $0 \leq a<b$

$$
\mu_{\kappa}\left(A_{a, b}\right)=\int_{A_{a, b}} \frac{1}{v_{\kappa}(r)}=\int_{a}^{b} J[1+T], \quad \int_{A_{a, b}} \frac{1-|\nabla r|^{2}}{v_{\kappa}(r)}=\int_{a}^{b} J T,
$$

where $J$ is the flux in (142), and

$$
T(s) \doteq \frac{\int_{\Gamma_{s}}|\nabla r|^{-1}}{\int_{\Gamma_{s}}|\nabla r|}-1
$$

Summarizing, in view of (152) and (153) we deduce from (150) the following inequalities:

$$
\begin{aligned}
\frac{\left\|\Delta u_{t, s}+\lambda u_{t, s}\right\|_{2}^{2}}{\left\|u_{t, s}\right\|_{2}^{2}} \leq & C\left(\frac{1}{\int_{t}^{s} J[1+T]}\left[F(t) \int_{t-1}^{S} J[1+T]+\int_{t-1}^{S} J T\right]\right. \\
& \left.+\frac{\int_{s}^{S} J[1+T]}{(S-s)^{2} \int_{t}^{s} J[1+T]}+\frac{\int_{t-1}^{t} J[1+T]}{\int_{t}^{s} J[1+T]}\right) \doteq \mathcal{Q}(t, s) .
\end{aligned}
$$

If we can guarantee that

$$
\liminf _{t \rightarrow+\infty} \liminf _{s \rightarrow+\infty} \frac{\left\|\Delta u_{t, s}+\lambda u_{t, s}\right\|_{2}^{2}}{\left\|u_{t, s}\right\|_{2}^{2}}=0
$$

then we are able to construct a sequence of approximating eigenfunctions for $\lambda$ as follows: fix $\varepsilon>0$. By (155) there exists a divergent sequence $\left\{t_{i}\right\}$ such that, for $i \geq i_{\varepsilon}$,

$$
\liminf _{s \rightarrow+\infty} \frac{\left\|\Delta u_{t_{i}, s}+\lambda u_{t_{i}, s}\right\|_{2}^{2}}{\left\|u_{t_{i}, s}\right\|_{2}^{2}}<\varepsilon / 2
$$


For $i=i_{\varepsilon}$, pick then a sequence $\left\{s_{j}\right\}$ realizing the liminf. For $j \geq j_{\varepsilon}\left(i_{\varepsilon}, \varepsilon\right)$

$$
\left\|\Delta u_{t_{i}, s_{j}}+\lambda u_{t_{i}, s_{j}}\right\|_{2}^{2}<\varepsilon\left\|u_{t_{i}, s_{j}}\right\|_{2}^{2}
$$

Writing $u_{\varepsilon} \doteq u_{t_{i_{\varepsilon}}, s_{j_{\varepsilon}}}$, by (156) from the set $\left\{u_{\varepsilon}\right\}$ we can extract a sequence of approximating eigenfunctions for $\lambda$, concluding the proof that $\lambda \in \sigma(M)$. To show (155), by (154) it is enough to prove that

$$
\liminf _{t \rightarrow+\infty} \liminf _{s \rightarrow+\infty} \mathcal{Q}(t, s)=0 .
$$

Suppose, by contradiction, that (157) were not true. Then, there exists a constant $\delta>0$ such that, for each $t \geq t_{\delta}, \liminf _{s \rightarrow+\infty} \mathcal{Q}(t, s) \geq 2 \delta$, and thus for $t \geq t_{\delta}$ and $s \geq s_{\delta}(t)$

$$
F(t) \int_{t-1}^{S} J[1+T]+\int_{t-1}^{S} J T+\int_{s}^{S} \frac{J[1+T]}{(S-s)^{2}}+\int_{t-1}^{t} J[1+T] \geq \delta \int_{t}^{s} J[1+T],
$$

and rearranging

$$
(F(t)+1) \int_{t-1}^{S} J[1+T]-\int_{t-1}^{S} J+\int_{s}^{S} \frac{J[1+T]}{(S-s)^{2}}+\int_{t-1}^{t} J[1+T] \geq \delta \int_{t}^{s} J[1+T]
$$

We rewrite the above integrals in order to make $\Theta(s)$ appear. Integrating by parts and using again the coarea's formula,

$$
\begin{aligned}
\int_{a}^{b} J[1+T] & =\int_{A_{a, b}} \frac{1}{v_{\kappa}(r)}=\int_{a}^{b} \frac{1}{v_{\kappa}(\sigma)}\left[\int_{\Gamma_{\sigma}} \frac{1}{|\nabla r|}\right] \mathrm{d} \sigma=\int_{a}^{b} \frac{\left(V_{\kappa}(\sigma) \Theta(\sigma)\right)^{\prime}}{v_{\kappa}(\sigma)} \mathrm{d} \sigma \\
& =\frac{V_{\kappa}(b)}{v_{\kappa}(b)} \Theta(b)-\frac{V_{\kappa}(a)}{v_{\kappa}(a)} \Theta(a)+\int_{a}^{b} \frac{V_{\kappa} v_{\kappa}^{\prime}}{v_{\kappa}^{2}} \Theta .
\end{aligned}
$$

To deal with the term containing the integral of $J$ alone in (159), we use the inequality $J(s) \geq \Theta(s)$ coming from the monotonicity formulae in Proposition 7.8. This passage is crucial for us to conclude. Inserting (160) and $J \geq \Theta$ into (159) we get

$$
\begin{gathered}
(F(t)+1) \frac{V_{\kappa}(S)}{v_{\kappa}(S)} \Theta(S)-(F(t)+1) \frac{V_{\kappa}(t-1)}{v_{\kappa}(t-1)} \Theta(t-1)+(F(t)+1) \int_{t-1}^{S} \frac{V_{\kappa} v_{\kappa}^{\prime}}{v_{\kappa}^{2}} \Theta \\
-\int_{t-1}^{S} \Theta+\frac{1}{(S-s)^{2}}\left[\frac{V_{\kappa}(S)}{v_{\kappa}(S)} \Theta(S)-\frac{V_{\kappa}(s)}{v_{\kappa}(s)} \Theta(s)+\int_{s}^{S} \frac{V_{\kappa} v_{\kappa}^{\prime}}{v_{\kappa}^{2}} \Theta\right]+\frac{V_{\kappa}(t)}{v_{\kappa}(t)} \Theta(t) \\
-\frac{V_{\kappa}(t-1)}{v_{\kappa}(t-1)} \Theta(t-1)+\int_{t-1}^{t} \frac{V_{\kappa} v_{\kappa}^{\prime}}{v_{\kappa}^{2}} \Theta \\
\geq \delta \frac{V_{\kappa}(s)}{v_{\kappa}(s)} \Theta(s)-\delta \frac{V_{\kappa}(t)}{v_{\kappa}(t)} \Theta(t)+\delta \int_{t}^{s} \frac{V_{\kappa} v_{\kappa}^{\prime}}{v_{\kappa}^{2}} \Theta .
\end{gathered}
$$

The idea to reach the desired contradiction is to prove that, as a consequence of (161),

$$
\int_{t-1}^{S} \Theta
$$

(hence, $\Theta(S)$ ) must grow faster as $S \rightarrow+\infty$ than the bound in (139). To do so, we need to simplify (161) in order to find a suitable differential inequality for (162).

We first observe that, both for $\kappa>0$ and for $\kappa=0$, there exists an absolute constant $\hat{c}$ such that $\hat{c}^{-1} \leq V_{\kappa} v_{\kappa}^{\prime} / v_{\kappa}^{2} \leq \hat{c}$ on $[1,+\infty)$. Furthermore, by the monotonicity of $\Theta$,

$$
\int_{s}^{S} \frac{V_{\kappa} v_{\kappa}^{\prime}}{v_{\kappa}^{2}} \Theta \leq \hat{c}(S-s) \Theta(S)
$$


Next, we deal with the two terms in the left-hand side of (161) that involve (162):

$$
\begin{aligned}
(F(t)+1) \int_{t-1}^{S} \frac{V_{\kappa} v_{\kappa}^{\prime}}{v_{\kappa}^{2}} \Theta-\int_{t-1}^{S} \Theta & =F(t) \int_{t-1}^{S} \frac{V_{\kappa} v_{\kappa}^{\prime}}{v_{\kappa}^{2}} \Theta+\int_{t-1}^{S} \frac{V_{\kappa} v_{\kappa}^{\prime}-v_{\kappa}^{2}}{v_{\kappa}^{2}} \Theta \\
& \leq \hat{c} F(t) \int_{t-1}^{S} \Theta+\int_{t-1}^{S} \frac{V_{\kappa} v_{\kappa}^{\prime}-v_{\kappa}^{2}}{v_{\kappa}^{2}} \Theta .
\end{aligned}
$$

The key point is the following relation:

$$
\frac{V_{\kappa}(s) v_{\kappa}^{\prime}(s)-v_{\kappa}(s)^{2}}{v_{\kappa}(s)^{2}} \begin{cases}=-1 / m & \text { if } \kappa=0 \\ \rightarrow 0 \text { as } s \rightarrow+\infty, & \text { if } \kappa>0 .\end{cases}
$$

Define

$$
\omega(t) \doteq \sup _{[t-1,+\infty)} \frac{V_{\kappa} v_{\kappa}^{\prime}-v_{\kappa}^{2}}{v_{\kappa}^{2}}, \quad \chi(t) \doteq \hat{c} F(t)+\omega(t) .
$$

Again by the monotonicity of $\Theta$,

$$
\begin{aligned}
(F(t)+1) \int_{t-1}^{S} \frac{V_{\kappa} v_{\kappa}^{\prime}}{v_{\kappa}^{2}} \Theta-\int_{t-1}^{S} \Theta & \leq[\hat{c} F(t)+\omega(t)] \int_{t-1}^{S} \Theta=\chi(t) \int_{t-1}^{S} \Theta \\
& \leq \chi(t) \Theta(t)+\chi(t) \int_{t}^{S} \Theta .
\end{aligned}
$$

For simplicity, hereafter we collect all the terms independent of $s$ in a function that we call $h(t)$, which may vary from line to line. Inserting (163) and (165) into (161) we infer

$$
\begin{aligned}
& {\left[\left(F(t)+1+\frac{1}{(S-s)^{2}}\right) \frac{V_{\kappa}(S)}{v_{\kappa}(S)}+\frac{\hat{c}}{S-s}\right] \Theta(S)+\chi(t) \int_{t}^{S} \Theta} \\
& \geq h(t)+\left(\delta+\frac{1}{(S-s)^{2}}\right) \frac{V_{\kappa}(s)}{v_{\kappa}(s)} \Theta(s)+\delta \hat{c}^{-1} \int_{t}^{s} \Theta .
\end{aligned}
$$

Summing $\delta \hat{c}^{-1}(S-s) \Theta(S)$ to the two sides of the above inequality, using the monotonicity of $\Theta$ and getting rid of the term containing $\Theta(s)$ we obtain

$$
\begin{aligned}
& {\left[\left(F(t)+1+\frac{1}{(S-s)^{2}}\right) \frac{V_{\kappa}(S)}{v_{\kappa}(S)}+\frac{\hat{c}}{S-s}+\delta \hat{c}^{-1}(S-s)\right] \Theta(S)+\chi(t) \int_{t}^{S} \Theta} \\
& \geq h(t)+\delta \hat{c}^{-1} \int_{t}^{S} \Theta .
\end{aligned}
$$

Using (164), the definition of $\chi(t)$ and the properties of $\omega(t), F(t)$, we can choose $t_{\delta}$ sufficiently large to guarantee that

$$
\delta \hat{c}^{-1}-\chi(t) \geq c_{\kappa} \doteq \begin{cases}\frac{1}{m}+\frac{\delta \hat{c}^{-1}}{2} & \text { if } \kappa=0, \\ \frac{\delta \hat{c}^{-1}}{2} & \text { if } \kappa>0,\end{cases}
$$

hence

$$
\left[\left(F(t)+1+\frac{1}{(S-s)^{2}}\right) \frac{V_{\kappa}(S)}{v_{\kappa}(S)}+\frac{\hat{c}}{S-s}+\delta \hat{c}^{-1}(S-s)\right] \Theta(S) \geq h(t)+c_{\kappa} \int_{t}^{S} \Theta .
$$

We now specify $S(s)$ depending on whether $\kappa>0$ or $\kappa=0$.

The case $\kappa>0$.

We choose $S \doteq s+1$. In view of the fact that $V_{\kappa} / v_{\kappa}$ is bounded above on $\mathbb{R}^{+},(169)$ becomes

$$
\bar{c} \Theta(s+1) \geq h(t)+c_{\kappa} \int_{t}^{s+1} \Theta \geq \frac{c_{\kappa}}{2} \int_{t}^{s+1} \Theta,
$$


for some $\bar{c}$ independent of $t, s$. Note that the last inequality is satisfied provided $s \geq s_{\delta}(t)$ is chosen to be sufficiently large, since the monotonicity of $\Theta$ implies that $\Theta \notin L^{1}\left(\mathbb{R}^{+}\right)$. Integrating and using again the monotonicity of $\Theta$, we get

$$
(s+1-t) \Theta(s+1) \geq \int_{t}^{s+1} \Theta \geq\left[\int_{t}^{s_{0}+1} \Theta\right] \exp \left\{\frac{c_{\kappa}}{2 \bar{c}}\left(s-s_{0}\right)\right\},
$$

hence $\Theta(s)$ grows exponentially. Ultimately, this contradicts our assumption (139).

The case $\kappa=0$.

We choose $S \doteq s+\sqrt{s}$. Since $V_{\kappa}(S) / v_{\kappa}(S)=S / m$, from (169) we infer

$$
\left[\left(F(t)+1+\frac{1}{s}\right) \frac{S}{m}+\frac{\hat{c}}{\sqrt{s}}+\delta \hat{c}^{-1} \sqrt{s}\right] \Theta(S) \geq h(t)+c_{\kappa} \int_{t}^{S} \Theta .
$$

Using the expression of $c_{\kappa}$ and the fact that $F(t) \rightarrow 0$, up to choosing $t_{\delta}$ and then $s_{\delta}(t)$ large enough we can ensure the validity of the following inequality:

$$
\left[\left(F(t)+1+\frac{1}{s}\right) \frac{S}{m}+\frac{\hat{c}}{\sqrt{s}}+\delta \hat{c}^{-1} \sqrt{s}\right]<\left[\frac{1}{m}+\frac{\delta \hat{c}^{-1}}{4}\right] S=\left[c_{\kappa}-\frac{\delta \hat{c}^{-1}}{4}\right] S
$$

for $t \geq t_{\delta}$ and $s \geq s_{\delta}(t)$. Plugging into (169), and using that $\Theta \notin L^{1}\left(\mathbb{R}^{+}\right)$,

$$
S \Theta(S) \geq h(t)+\frac{c_{\kappa}}{c_{\kappa}-\delta \hat{c}^{-1} / 4} \int_{t}^{S} \Theta \geq(1+\varepsilon) \int_{t}^{S} \Theta,
$$

for a suitable $\varepsilon>0$ independent of $t, S$, and provided that $S \geq s_{\delta}(t)$ is large enough. Integrating and using again the monotonicity of $\Theta$,

$$
S \Theta(S) \geq(S-t) \Theta(S) \geq \int_{t}^{S} \Theta \geq\left[\int_{t}^{S_{0}} \Theta\right]\left(\frac{S}{S_{0}}\right)^{1+\varepsilon},
$$

hence $\Theta(S)$ grows polynomially at least with power $\varepsilon$, contradicting (139).

Concluding, both for $\kappa>0$ and for $\kappa=0$ assuming (158) leads to a contradiction with our assumption (139), hence (155) holds, as required.

\section{REFERENCES}

[1] V. L. Ahlfors, Sur le type d'une surface de Riemann, C.R. Acad. Sci. Paris, 201 (1935) 30-32.

[2] A. Alarcón, Compact complete minimal immersions in $\mathbb{R}^{3}$. Trans. Amer. Math. Soc. 362 (2010), 4063-4076.

[3] A. Alarcón, L. Ferrer and F. Martin, Density theorems for complete minimal surfaces in $\mathbb{R}^{3}$. Geom. Funct. Anal. 18 (2008), no. 1, 1-49.

[4] A. Alarcón, F. J. López, Null curves in $\mathbb{C}^{3}$ and Calabi-Yau conjectures. Math. Ann. 355, (2013), no. 2, 429-455.

[5] M. T. Anderson, The compactication of a minimal submanifold in Euclidean space by the Gauss map. I.H.E.S. Preprint, 1984. Available online at http://www.math.sunysb.edu/ anderson/compactif.pdf

[6] M. T. Anderson, Complete minimal varieties in hyperbolic space. Invent. Math. 69, (1982), no. 3, 477-494.

[7] P. Andrade, A wild minimal plane in $\mathbb{R}^{3}$. Proc. Amer. Math. Soc. 128 (2000), no. 5, 1451-1457.

[8] A. Baider, Noncompact Riemannian manifolds with discrete spectra, J. Diff. Geom. 14 (1979), $41-57$.

[9] R. Bañuelos and T. Carroll, Brownnian motion and the fundamental frequence of a drum. Duke Math. J. 75 (1994), 575-602.

[10] C. Bär, G. P. Bessa, Stochastic completeness and volume growth.Proc. Amer. Math. Soc. 138 (2010), no. 7, 2629-2640.

[11] J. L. Barbosa, G. P. Bessa, and J. F. Montenegro, On Bernstein-Heinz-Chern-Flanders inequalities. Math. Proc. Camb. Phil. Soc. 144 (2008) 457-464.

[12] J. L. Barbosa, K. Kenmotsu and O. Oshikiri, Foliations by hypersurfaces with constant mean curvature. Math. Z. 207 (1991), 97-108

[13] J. Barta, Sur la vibration fundamentale d'une membrane. C. R. Acad. Sci. 204, (1937), 472-473. 
[14] E. Beltrami, Delle variabili complesse sopra una superficie qualunque. Ann. Mat. Pura Appl. (2) 1 (1867), 329-366.

[15] M. Berger, P. Gauduchon and E. Mazet, Le spectre d'une varit riemannienne. (French) Lecture Notes in Mathematics, Vol. 194 Springer-Verlag, Berlin-New York 1971 vii+251 pp.

[16] G. P. Bessa, S. M. Costa, S.M., On submanifolds with tamed second fundamental form. Glasg. Math. J. 51, (2009), no. 3, 669-680.

[17] G. P. Bessa, L. P. Jorge and L. Mari, On the spectrum of bounded immersions. J. Differential Geom. 99, (2) (2015), 215-253.

[18] G. P. Bessa, L. P. Jorge, J. F. Montenegro, Complete submanifolds of $R^{n}$ with finite topology. Comm. Anal. Geom. 15, (2007), no. 4, 725-732.

[19] G. P. Bessa, L. P. Jorge and J. F. Montenegro, The Spectrum of the Martin-Morales-Nadirashvili Minimal Surfaces is discrete. J. Geom. Anal. 20 (2010), 63-71.

[20] G. P. Bessa, L. P. Jorge and G. Oliveira-Filho Half-Space Theorems for Minimal Surfaces with Bounded Curvature. J. Differential Geometry 57 (2001), 493-508.

[21] G. P. Bessa and J. F. Montenegro, Eigenvalue estimates for submanifolds with locally bounded mean curvature. Ann. Global Anal. and Geom.24 (2003), 279-290.

[22] G. P. Bessa and J. F. Montenegro, An extension of Barta's Theorem and geometric applications. Ann. Global Anal. and Geom. 31, (2007) 345-362.

[23] G. P. Bessa and J. F. Montenegro, On Cheng's eigenvalue comparison theorem. Math. Proc. Camb. Phil. Soc. 144, (2008) 673-682.

[24] G. P. Bessa and J. F. Montenegro, Mean time exit and isoperimetric inequalities for minimal submanifolds of $N \times \mathbb{R}$. Bull. Lond. Math. Soc. 41 (2009), 242-252.

[25] G. P. Bessa, J. F. Montenegro and P. Piccione, Riemannian submersions with discrete spectrum. J. Geom. Anal. 22 (2012), no. 2, 603-620.

[26] B. Bianchini, L. Mari and M. Rigoli, On some aspects of oscillation theory and geometry. Mem. Amer. Math. Soc. 255, no. 1056, (2013), vi+195pp.

[27] R. L. Bishop, R. J. Crittenden, Geometry of manifolds. Pure and Applied Mathematics, Vol. XV Academic Press, New York-London (1964) ix+273 pp.

[28] M. Bordoni, Spectral estimates for submersions with fibers of basic mean curvature, An. Univ. Vest Timiş. Ser. Mat.-Inform. 44, no. 1, (2006), 23-36.

[29] R. Brooks, A relation between growth and the spectrum of the Laplacian. Math. Z. 178 (1981), no. $4,501-508$

[30] E. Calabi, Problems in Differential Geometry. (S. Kobayashi and J. Eells, Jr., eds.) Proc. of the United States-Japan Seminar in Differential Geometry, Kyoto, Japan, 1965, Nippon Hyoronsha Co. Ltd., Tokyo (1966) 170.

[31] P. Castillon, Spectral properties of constant mean curvature submanifolds in hyperbolic space. Ann. Global Anal. Geom. 17, (1999), no.6, 563-580.

[32] N. Charalambous, Z. Lu, On the spectrum of the Laplacian. Math. Ann. 359, (2014), no.1-2, 211-238.

[33] I. Chavel, Eigenvalues in Riemannian geometry. Including a chapter by Burton Randol. With an appendix by Jozef Dodziuk. Pure and Applied Mathematics, 115. Academic Press, Inc., Orlando, FL, 1984. xiv+362 pp. ISBN: 0-12-170640-0.

[34] J. Cheeger, A lower bound for the smallest eigenvalue of the Laplacian. Problems in analysis. (Papers dedicated to Salomon Bochner, 1969), pp. 195-199. Princeton Univ. Press, Princeton, N. J., 1970.

[35] Q. Chen, On the area growth of minimal surfaces in $H^{n}$. Geom. Dedicata 75, (1999), no.3, 263-273.

[36] Q. Chen, Y. Cheng, Chern-Osserman inequality for minimal surfaces in $H^{n}$. Proc. Amer. Math. Soc. 128, (2000), no.8, 2445-2450.

[37] J. Cheeger, D. Ebin, Comparison theorems in Riemannian geometry. North-Holland Mathematical Library, Vol. 9. North-Holland Publishing Co., Amsterdam-Oxford; American Elsevier Publishing Co., Inc., New York, 1975. viii+174 pp.

[38] S. Y. Cheng, Eigenvalue comparison theorems and its geometric applications. Math. Z. 143 (1975), 289-297.

[39] S. Y. Cheng, Eigenfunctions and eigenvalues of the Laplacian. Am. Math. Soc. Proc. Sym. Pure Math. 27 (1975), 185-193.

[40] S. Y. Cheng, P; Li, and S. T. Yau, Heat equations on minimal submanifolds and their applications. Amer. J. Math. 106, (1984), 1033-1065.

[41] Leung-Fu Cheung, Pui-Fai Leung, Eigenvalue estimates for submanifolds with bounded mean curvature in the hyperbolic space. Math. Z. 236, (2001), no. 3, 525-530.

[42] S. Y. Cheng and S. T. Yau, Differential equations on Riemannian manifolds and their geometric applications. Comm. Pure Appl. Math. 27 (1975), 333-354. 
[43] X. Cheng, D. Zhou, Eigenvalues of the drifted Laplacian on complete metric measure spaces. Available on arXiv:1305.4116.

[44] S. S. Chern, The geometry of G-structures. Bull. Amer. Math. Soc. 72 (1966), 167-219.

[45] S. S. Chern, R. Osserman, Complete minimal surface in $E^{n}$. J. dAnalyse Math. 19, (1967), 15-34.

[46] S. S. Chern, R. Osserman, Complete minimal surfaces in euclidean space. Academic Press, New York (1984).

[47] T. H. Colding, W. P. Minicozzi, The Calabi-Yau conjectures for embedded surfaces. Ann. of Math. (2) $\mathbf{1 6 7}$, no. 1 (2008), 211-243.

[48] P. Collin, H. Rosenberg, Notes sur la démonstration de N. Nadirashvili des conjectures de Hadamard et Calabi-Yau. Bull. Sci. Math. 123, no. 7, (1999), 563-575.

[49] E. B. Davies Heat kernels and spectral theory. Cambridge Tracts in Mathematics, 92. Cambridge University Press, Cambridge, 1989. x+197 pp. ISBN: 0-521-36136-2.

[50] E. B. Davies, Spectral theory and differential operators. Cambridge Studies in Advanced Mathematics, 42. Cambridge University Press, Cambridge, 1995. x+182 pp. ISBN: 0-521-47250-4

[51] M. DoCarmo, C. K. Peng, Stable complete minimal surfaces in $\mathbb{R}^{3}$ are planes. Bull. Amer. Math. Soc. (N.S.) 1, no. 6 (1979), 903-906.

[52] H. Donnelly, On the essential spectrum of a complete Riemannian manifold, Topology 20, no. 1, (1981), 1-14.

[53] H. Donnelly and P. Li, Pure point spectrum and negative curvature for non-compact manifolds.Duke Math. J. 46 (1979), 497-503.

[54] H. Donnelly, Negative curvature and embedded eigenvalues. Math. Z. 203, (1990), 301-308.

[55] H. Donnelly, Exhaustion functions and the spectrum of Riemannian manifolds. Indiana Univ. Math. J. 46, (1997), no. 2, 505-527.

[56] H. Donnelly, N. Garofalo, Riemannian manifolds whose Laplacian have purely continuous spectrum. Math. Ann. 293, (1992), 143-161, MR1162679, Zbl 0735.58033.

[57] J. Douglas, Solution of the problem of Plateau. Trans. Amer. Math. Soc. 33 (1931), 263-321.

[58] J. Douglas, The least area property of the minimal surface determined by an arbitrary Jordan contour. Proc. Natl. Acad. Sci. U. S. A. 17, (1931), no.4, 211-216.

[59] E. B. Dynkin, Markov processes. Vols. I, II. Translated with the authorization and assistance of the author by J. Fabius, V. Greenberg, A. Maitra, G. Majone. Die Grundlehren der Mathematischen Wissenschaften, Bände 121, 122 Academic Press Inc., Publishers, New York; Springer-Verlag, Berlin-Göttingen-Heidelberg 1965 Vol. I: xii+365 pp.; Vol. II: viii+274 pp.

[60] K. D. Elworthy, F-Y. Wang, F-Y. Essential spectrum on Riemannian manifolds. Recent Developments in Stochastic Analysis and Related Topics, World Sci. Publ., Hackensack, NJ, 151-165 (2004).

[61] J. F. Escobar, On the spectrum of the Laplacian on complete Riemannian manifolds. Comm. Partial Differ. Equations 11, (1985), 63-85.

[62] J. F. Escobar, A. Freire, A., The spectrum of the Laplacian on manifolds of positive curvature. Duke Math. J. 65, (1992), 1-21.

[63] L. Ferrer, F. Martin, W. Meeks III, Existence of proper minimal surfaces of arbitrary topological type. Adv. Math. 231, no. 1, (2012), 378-413.

[64] D. Fischer-Colbrie R. Schoen, The structure of complete stable minimal surfaces in 3-manifolds of nonnegative scalar curvature. Comm. Pure Appl. Math. 33 (1980), 1999-211.

[65] P. Freitas, J. Mao, Jing and I. Salavessa, Spherical symmetrization and the first eigenvalue of geodesic disks on manifolds. Calc. Var. Partial Differential Equations 51 (2014), no. 3-4, 701-724.

[66] M. P. Gaffney, A special Stokes theorem for complete Riemannian manifolds. Ann. of Math. 60 (1954), 140-145.

[67] I. M. Glazman, Direct methods of qualitative spectral analysis of singular differential operators. Translated from the Russian by the IPST staff Israel Program for Scientific Translations, Jerusalem, 1965; Daniel Davey \& Co., Inc., New York 1966 ix+234 pp.

[68] A. Gray, Pseudo-Riemannian almost product manifolds and submersions, J. Math. Mech. 16 (1967), 715-737.

[69] R. E. Greene, H. Wu, Function theory on manifolds which possess a pole. Lecture Notes in Mathematics, 699. Springer, Berlin, 1979. ii+215 pp. ISBN: 3-540-09108-4

[70] A. Grigor'yan, Set of positive solutions of Laplace-Beltrami equation on special type of Riemannian manifolds, (in Russian) Izv. Vyssh. Uchebn. Zaved., Matematika, (1987) no.2, 30-37. Engl. transl. Soviet Math (Iz.VUZ), 31 (1987) no.2, 48-60.

[71] A. Grigorýan, Analytic and geometric background of recurrence and non-explosion of the Brownian motion on Riemannian manifolds. Bull. Amer. Math. Soc. (N.S.) 36 (1999), no. 2, 135-249.

[72] A. Grigor'yan, Heat kernels on weighted manifolds and applications, The ubiquitous heat kernel, Contemp. Math., vol. 398, Amer. Math. Soc., Providence, RI, 2006, pp. 93-191. 
[73] A. Grigorýan, Heat kernel and analysis on manifolds. AMS/IP Studies in Advanced Mathematics, 47. American Mathematical Society, Providence, RI; International Press, Boston, MA, 2009. xviii+482 pp. ISBN: 978-0-8218-4935-4.

[74] A. Grigorýan, Jiaxin Hu, and Ka-Sing Lau, Heat kernels on metric spaces with doubling measure, Fractal geometry and stochastics IV, Progr. Probab., vol. 61, Birkhäuser Verlag, Basel, 2009, pp. 3-44.

[75] M. Gromov, Metric structures for Riemannian and non-Riemannian spaces. Based on the 1981 French original. With appendices by M. Katz, P. Pansu and S. Semmes. Translated from the French by Sean Michael Bates. Reprint of the 2001 English edition. Modern Birkhuser Classics. Birkhuser Boston, Inc., Boston, MA, 2007. xx+585 pp. ISBN: 978-0-8176-4582-3; 0-8176-4582-9.

[76] J. Hadamard, Les surfaces à courbures opposées et leurs linges géodésiquese. J. Math. Pures Appl. 4 (1898), 27-73.

[77] W. Haymann. Some bounds for principal frequency. Applicable Anal. 7 (1978), 247-254.

[78] M. Harmer, Discreteness of the spectrum of the Laplacian and Stochastic incompleteness. J. Geom. Anal. 19 (2009), 358-372.

[79] Y. Higuchi, A remark on exponential growth and the spectrum of the Laplacian. Kodai Math. J. 24, (2001), 42-47.

[80] K. Hoffman, Banach spaces of analytic functions. Reprint of the 1962 original. Dover Publications, Inc., New York, 1988. viii+216 pp. ISBN: 0-486-65785-X.

[81] K. Ichihara, Curvature, geodesics and the Brownian motion on a Riemannian manifold. II Recurrence properties, Nagoya Math. J., 87 (1982) 115-125.

[82] L. P. Jorge, and D. Koutrofiotis, An estimate for the curvature of bounded submanifolds. Amer. J. Math., 103, no. 4, 1980, 711-725.

[83] L. P. Jorge and F. Tomi, The barrier principle for minimal submanifolds of arbitrary codimension. Ann. Global Anal. Geom. 24 (2003), no. 3, 261-267.

[84] L. P. Jorge and F. Xavier, A complete minimal surface in $\mathbb{R}^{3}$ between two parallel planes. Annals of Math. (2) 112 (1980), 203-206.

[85] L. Karp, Noncompact manifolds with purely continuous spectrum. Mich. Math. J. 31, (1984), 339347.

[86] R. Kleine, Discreteness conditions for the Laplacian on complete, noncompact Riemannian manifolds. Math. Z. 198 (1988), no. 1, 127-141.

[87] R. Kleine, Warped products with discrete spectra. Results Math. 15 (1989), no. 1-2, 81-103.

[88] H. Kumura, On the essential spectrum of the Laplacian on complete manifolds. J. Mat. Soc. Japan 49, (1997), no. 1, 1-14 . (1997).

[89] H. Kumura, On the essential spectrum of the Laplacian and vague convergence of the curvature at infinity. Comm. P. Diff. Eq. 30, (2005), no. 10-12, 1555-1565.

[90] J. Li, Spectrum of the Laplacian on a complete Riemannian manifold with non-negative Ricci curvature which possess(es) a pole. J. Math. Soc. Japan 46, (1994), 213-216.

[91] B. P. Lima, L. Mari, J. F. Montenegro, F. B. Vieira, Density and spectrum of minimal submanifolds in space forms. Math. Ann (Online first), (2016), 1-32.

[92] F. Lopez, F. Martin, and S. Morales, Adding handles to Nadirashvili's surfaces. J. Diff. Geom. 60 (2002), no. 1, 155-175.

[93] F. Lopez, F. Martin, and S. Morales, Complete nonorientable minimal surfaces in a ball of $\mathbb{R}^{3}$. Trans. Amer. Math. Soc. 358, no. 9, (2006), 3807-3820.

[94] J. Lott, On the spectrum of a finite-volume negatively-curved manifold. Amer. J. Math. 123, (2001), no. 2, 185-205.

[95] Z. Lu, D. Zhou, On the essential spectrum of complete non-compact manifolds. J. Funct. Anal. 260, (2011), no. 11, 3283-3298.

[96] E. Makai, A lower estimation of the principal frequencies of simply connected membranes. Acta Math. Acad. Sci. Hungar. 16 (1965), 319-366.

[97] Steen Markvorsen, On the mean exit time from a minimal submanifold, J. Differential Geometry 29 (1989), 1-8.

[98] F. Martín, W. Meeks III, N. Nadirashvili, Bounded domains which are universal for minimal surfaces. Amer. J. Math. 129 (2007), no. 2, 455-461

[99] F. Martín, S. Morales, Complete proper minimal surfaces in convex bodies of $\mathbb{R}^{3}$. Duke Math. J. 128 (2005), 559-593.

[100] F. Martín, S. Morales, Complete proper minimal surfaces in convex bodies of $\mathbb{R}^{3}$. II. The behavior of the limit set. Comment. Math. Helv. 81 (2006), 699-725.

[101] F. Martín, S. Morales, Construction of a complete bounded minimal annulus in $\mathbb{R}^{3}$. (Spanish) Proceedings of the Meeting of Andalusian Mathematicians, Vol. II (Spanish) (Sevilla, 2000), 649653, Colecc. Abierta, 52, Univ. Sevilla Secr. Publ., Seville, 2001. 
[102] F. Martín, N. Nadirashvili, A Jordan curve spanned by a complete minimal surface. Arch. Ration. Mech. Anal. 184 (2007), no. 2, 285-301.

[103] R. B. Matos, F. F. Montenegro, Spectrum of the Laplacian on radial graphs. Available on arXiv:1303.3293.

[104] H. P. McKean, An upper bound to the spectrum of $\Delta$ on a manifold of negative curvature. J. Differential Geometry 4 (1970) 359-366.

[105] P. Mattila, Geometry of sets and measures in Euclidean spaces. Fractals and rectifiability. Cambridge Studies in Advanced Mathematics, 44. Cambridge University Press, Cambridge, 1995. xii+343 pp. ISBN: 0-521-46576-1.

[106] L. A. Monte, J. F. Montenegro, Essential spectrum of a class of Riemannian manifolds. J. Geom. Anal. 25, (2015), no. 4, 2241-2261.

[107] N. Nadirashvili, Hadamard's and Calabi-Yau's conjectures on negatively curved and minimal surfaces. Invent. Math. 126 (1996), 457-465.

[108] G. Oliveira-Filho, Compactification of minimal submanifolds of hyperbolic space. Comm. Anal. Geom. 1, (1993), no.1, 1-29.

[109] B. O'Neill, The fundamental equations of a submersion, Michigan Math. J. 13 (1966), 459-469.

[110] B. O'Neill, Submersions and geodesics, Duke Math. J. 34 (1967), 363-373.

[111] W. F. Osgood, A Jordan curve of positive area. Trans. Amer. Math. Soc. 4 (1903), no.1, 107-112.

[112] R. Osserman. A note on Hymann's theorem on the bass note of a drum. Comment. Math. Helv. 52 (1977), 545-555.

[113] Vicente Palmer, Isoperimetric inequalities for extrinsic balls in minimal submanifolds and their applications, J. Lond. Math. Soc. 60 (1999), no.2 607-616.

[114] A. Persson, Bounds for the discrete part of the spectrum of a semi-bounded operator. Math. Scand. 8 (1960), 143-153.

[115] P. Petersen, . Riemannian geometry. Second edition. Graduate Texts in Mathematics, 171. Springer, New York, 2006. xvi+401 pp. ISBN: 978-0387-29246-5; 0-387-29246-2.

[116] S. Pigola, M. Rigoli and A. G. Setti, Vanishing and finiteness results in Geometric Analysis. A generalization of the Böchner technique. Progress in Math. 266, Birkäuser, 2008.

[117] A. V. Pogorelov, On the stability of minimal surfaces. (Russian) Dokl. Akad. Nauk SSSR 260 (1981), no. 2, 293-295.

[118] T. Radó, On the Problem of Plateau Ann. of Math. (2) 31 no. 3, (1930), 457-469.

[119] F. Rellich, Über das asymptotische Verhalten der Lsungen von $\Delta u+\lambda u=0$ in unendlichen Gebieten. (German) Jber. Deutsch. Math. Verein. 53, (1943). 57-65.

[120] H. Rosenberg, Intersection of minimal surfaces of bounded curvature. Bull. Sci. Math. 125 (2) (2001), 161-168.

[121] H. Rosenberg, E. Toubiana, A cylindrical type complete minimal surface in the slab of $\mathbb{R}^{3}$. Bull. Sci. Math. (2 ) 111, no. 3, (1987), 241-245.

[122] A. Ros, One-sided complete stable minimal surfaces., J. Diff. Geom. 74 (2006), 69-92.

[123] R. Schoen, Estimates for stable minimal surfaces in three dimensional manifolds. Seminar on minimal submanifolds, 111-126, Ann. of Math. Stud., 103, Princeton Univ. Press, Princeton, NJ, (1983).

[124] R. Schoen, H. Tran, Complete manifolds with bounded curvature and spectral gaps. Avalable at arXiv:1510.05046.

[125] R. Schoen and S. T. Yau, Lectures on differential geometry. Conference Proceedings and Lecture Notes in Geometry and Topology, I. International Press, Cambridge, MA, 1994. v+235 pp. ISBN: 1-57146-012-8.

[126] P. Scott, The geometries of 3-manifolds, Bull. London Math. Soc. 15 (5) (1983), 401-487.

[127] R. S. Sena, R.S., a note on the spectrum of self-shrinkers. Preprint.

[128] L. Silvares, On the essential spectrum of the Laplacian and the drifted Laplacian. J. Funct. Anal. 266, (2014) no. 6, 3906-3936.

[129] Simon, L., Lectures on geometric measure theory. Proceedings of the Centre for Mathematical Analysis, Australian National University, 3. Australian National University, Centre for Mathematical Analysis, Canberra. vii+272 pp (1983)

[130] K. T. Sturm, On the $L^{p}$-spectrum of uniformly elliptic operators on Riemannian manifolds. J. Funct. Anal. 118, (1993), no. 2, 442-453.

[131] T. Tayoshi, On the spectrum of the Laplace-Beltrami operator on noncompact surface, Proc. Japan Acad. 47, (1971), 579-585.

[132] M. Tokuomaru, Complete minimal cylinders properly immersed in the unit ball. Kyushu J. math. 61 (2007), no. 2, 373-394, MR2362891, Zbl 1143.53010.

[133] V. G. Tkachev, Finiteness of the number of ends of minimal submanifolds in Euclidean space. Manuscripta Math. 82, (1994), 313-330. 
[134] F. Torralbo, Rotationally invariant constant mean curvature surfaces in homogeneus 3-manifolds, Differential Geom. Appl. 28 no. 5 (2010), 593-607.

[135] A. Wetzler, Y. Aflalo, A. Dubrovina and R. Kimmel, The Laplace-Beltrami Operator: A Ubiquitous Tool for Image and Shape Processing. Mathematical Morphology and Its Applications to Signal and Image Processing Lecture Notes in Computer Science Volume 7883, 2013, 302-316

[136] H. Whitney, Geometric Integration Theory. Princeton Mathematical Series, 1957, xv+387 pp., Princeton University Press, Princeton, N. J., 1957.

[137] J.Wang, The spectrum of the Laplacian on a manifold of nonnegative Ricci curvature. Math. Res. Lett. 4, (1997) no. 4, 473-479.

[138] S. T. Yau, Review of Geometry and Analysis. Kodaira's issue. Asian J. Math. 4 (2000), $235-278$.

[139] S. T. Yau, Review of Geometry and Analysis. Mathematics: frontier and perspectives. Amer. Math. Soc. Providence. RI. (2000), 353-401.

[140] S.T. Yau, Nonlinear analysis in geometry. Monographies de L'Enseignement Mathmatique [Monographs of L'Enseignement Mathmatique], 33. Srie des Confrences de l'Union Mathmatique Internationale [Lecture Series of the International Mathematics Union], 8. L'Enseignement Mathmatique, Geneva, 54 pp (1986).

[141] D. Zhou, Essential spectrum of the Laplacian on manifolds of nonnegative curvature. Int. Math. Res. Not. (1994), no. 5, 209 ff. 